\title{
406.
}

\section{ON THE CURVES WHICH SATISFY GIVEN CONDITIONS.}

[From the Philosophical Transactions of the Royal Society of London, vol. CLVIII. (for the year 1868), pp. 75-143. Received April 18,-Read May 2, 1867.]

The present Memoir relates to portions only of the subject of the curves which satisfy given conditions; but any other title would be too narrow: the question chiefly considered is that of finding the number of the curves which satisfy given conditions; the curves are either curves of a determinate order $r$ (and in this case the conditions chiefly considered are conditions of contact with a given curve), or else the curves are conics; and here (although the conditions chiefly considered are conditions of contact with a given curve or curves) it is necessary to consider more than in the former case the theory of conditions of any kind whatever. As regards the theory of conics, the Memoir is based upon the researches of Chasles and Zeuthen, as regards that of the curves of the order $r$, upon the researches of De Jonquières: the notion of the quasi-geometrical representation of conditions by means of loci in hyper-space is employed by Salmon in his researches relating to the quadric surfaces which satisfy given conditions. The papers containing the researches referred to are included in the subjoined list. I reserve for a separate Second Memoir the application to the present question, of the Principle of Correspondence.

List of Memoirs and Works relating to the Curves which satisfy given conditions, with remarks.

De Jonquières: “Théorèmes généraux conceruant les courbes géométriques planes d'un ordre quelconque," Liouv. t. VI. (1861), pp. 113-134. In this valuable memoir is established the notion of a series of curves of the index $N$; viz. considering the curves of the order $n$ which satisfy $\frac{1}{2} n(n+3)-1$ conditions, then if $N$ denotes how many there are of these curves which pass through a given arbitrary point, the series is said to be of the index $N$.

In Lemma IV it is stated that all the curves $C_{n}$ of a series of the index $N$ can 
be analytically represented by an equation $F(y, x)=0$, which is rational and integral of the degree $N$ in regard to a variable parameter $\lambda$ : this is not the case; see Annex No. 1.

Chasles: Various papers in the Comptes Rendus, t. LvIII. et seq. 1864-67. The first of them (Feb. 1864), entitled "Détermination du nombre des sections coniques qui doivent toucher cinq courbes données d'ordre quelconque, ou satisfaire à diverses autres conditions," establishes the notion of the two characteristics $(\mu, \nu)$ of a system of conics which satisfy four conditions; viz. $\mu$ is the number of these conics which pass through a given arbitrary point, and $\nu$ the number of them which touch a given arbitrary line. The Principle of Correspondence for points on a line is established in the paper of June-July 1864. Many of the leading points of the theory are reproduced in the present Memoir. The series of papers includes one on the conics in space which satisfy seven conditions (Sept. 1865), and another on the surfaces of the second order which satisfy eight conditions (Feb. 1866).

Salmon: "On some Points in the Theory of Elimination," Quart. Math. Journ. t. vII. pp. 327-337 (Feb. 1866); "On the Number of Surfaces of the Second Degree which can be described to satisfy nine Conditions," Ibid. t. viII. pp. $1-7$ (June 1866), which two papers are here referred to on account of the notion which they establish of the quasi-geometrical representation of conditions by means of loci in hyper-space.

Zeuthen: Nyt Bidrag... Contribution to the Theory of Systems of Conics which satisfy four conditions, $8^{\circ}$. pp. 1-97 (Copenhagen, Cohen, 1865), translated, with an addition, in the Nouvelles Annales.

The method employed depends on the determination of the line-pairs and pointpairs, and of the numerical coefficients by which these have to be multiplied, in the several systems of conics which satisfy four conditions of contact with a given curve or curves. It is reproduced in detail, with the enumeration called "Zeuthen's Capitals," in the present Memoir.

Cayley: "Sur les coniques déterminées par cinq conditions d'intersection avec une courbe donnée," Comptes Rendus, t. LXIII. pp. 9-12, July 1866. Results reproduced in the present Memoir.

De Jonquières: Two papers, Comptes Rendus, t. LxIII. Sept. 1866, reproduced and further developed in the "Mémoire sur les contacts multiples d'ordre quelconque des courbes du degré $r$ qui satisfont à des conditions données de contact avec une courbe fixe du degré $m$; suivi de quelques réflexions sur la solution d'un grand nombre de questions concernant les propriétés projectives des courbes et des surfaces algébriques," Crelle, t. LXvi. (1866), pp. 289-322,-contain a general formula for the number of curves $C^{r}$ having contacts of given orders $a, b, c, \ldots$ with a given curve $U^{m}$, which formula is referred to and considered in the present Memoir.

De Jonquières: Recherches sur les séries ou systèmes de courbes et de surfaces algébriques d'ordre quelconque; suivies d'une réponse \&c. 4. Paris, Gauthier Villars, $18666^{(1)}$.

1 The foregoing list is not complete, and the remarks are not intended to give even a sketch of the contents of the works comprised therein, but only to show their bearing on the present Memoir. 


\section{Article Nos. 1 to 23.-On the quasi-geometrical representation of Conditions.}

1. A condition imposed upon a subject gives rise to a relation between the parameters of the subject; for instance, the subject may be, as in the present Memoir, a plane curve of a given order, and the parameters be any arbitrary parameters contained in the equation of the curve. The condition may be onefold, twofold,... or, generally, $k$-fold, and the corresponding relation is onefold, twofold, ... or $k$-fold accordingly. Two or more conditions, each of a given manifoldness, may be regarded as forming together a single condition of a higher manifoldness, and the corresponding relations as forming a single relation; and thus, though it is often convenient to consider two or more conditions or relations, this case is in fact included in that of a $k$-fold condition or relation. In dealing with such a condition or relation it is assumed that the number of parameters is at least $=k$; for otherwise there would not in general be any subject satisfying the condition: when the number of parameters is $=k$, the number of subjects satisfying the condition is in general determinate.

2. A subject which satisfies'a given condition may for shortness be termed a solution of the condition; and in like manner any set of values of the parameters satisfying the corresponding relation may be termed a solution of the relation. Thus for a $k$-fold condition or relation, and the same number $k$ of parameters, the number of solutions is in general determinate.

3. A condition may in some cases be satisfied in more than a single way, and if a certain way be regarded as the ordinary and proper one, then the others are special or improper: the two epithets may be used conjointly, or either of them separately, almost indifferently. For instance, the condition that a curve shall touch a given curve (have with it a two-pointic intersection) is satisfied if the curve have with the given curse a proper contact; or if it have on the given curve a node or a cusp (or, more specially, if it be or comprise as part of itself two coincident curves); or if it pass through a node or a cusp of the given curve: the first is regarded as the ordinary and proper way of satisfying the condition; the other two as special or improper ways; and the corresponding solutions are ordinary and proper solutions, or special or improper ones accordingly. This will be further explained in speaking of the locus which serves for the representation of a condition.

4. A set of any number, say $\omega$, of parameters may be considered as the coordinates of a point in $\omega$-dimensional space; and if the parameters are connected by a onefold, twofold, $\ldots$ or $k$-fold relation, then the point is situate on a onefold, twofold, ... or $k$-fold locus accordingly; to the relation made up of two or more relations corresponds the locus which is the intersection or common locus of the loci corresponding to the several component relations respectively. A locus is at most $\omega$-fold, viz. it is in this case a point-system. The relation made up of a $k$-fold relation, an $l$-fold relation, \&c., is in general $(k+l+\& \mathrm{c}$.) fold, and the corresponding locus is $(k+l+\& \mathrm{c}$. $)$ fold accordingly.

5. The order of a point-system is equal to the number of the points thereof, where, of course, coincident points have to be attended to, so that the distinct points of the system may have to be reckoned each its proper number of times. The locus

C. VI. 
corresponding to any linear $j$-fold relation between the coordinates is said to be a $j$-fold omal locus; and if to any given $k$-fold relation we join an arbitrary $(\omega-k)$ fold linear relation, that is, intersect the $k$-fold locus by an arbitrary $(\omega-k)$ fold omal locus, so as to obtain a point-system, the order of the $k$-fold relation or locus is taken to be equal to the number of points of the point-system, that is, to the order of the pointsystem. And this being so, if a $k$-fold relation, an $l$-fold relation, \&c. are completely independent, that is, if they are not satisfied by values which satisfy a less than $(k+l+\& \mathrm{c}$.) fold relation, or, what is the same thing, if the $k$-fold locus, the $l$-fold locus, \&c., have no common less than $(k+l+\&$ c.) fold locus, then the relations make up together a $(k+l+\& c$.$) fold relation, and the loci intersect in a (k+l+\& c$ c.) fold locus, the orders whereof are respectively equal to the product of the orders of the given relations or loci. In particular if we have $k+l+\& c .=\omega$, then we have an $\omega$-fold relation, and corresponding thereto a point-system, the orders whereof are respectively equal to the product of the orders of the given relations or loci.

6. A $k$-fold relation, an $l$-fold relation, \&c., if they were together equivalent to a less than $(k+l+\&$ c.) fold relation, would not be independent; but the relations, assumed to be independent, may yet contain a less than $(k+l+\& c$.) fold relation, that is, they may be satisfied by the values which satisfy a certain less than $(k+l+\&$ c.) fold relation (say the common relation), and exclusively of these, only by the values which satisfy a proper $(k+l+\&$ c. $)$ fold relation, which is, so to speak, a residual equivalent of the given relations. This is more clearly seen in regard to the loci; the $k$-fold locus, the $l$-fold locus, \&c. may have in common a less than $(k+l+\& c$ c. ) fold locus, and besides intersect in a residual $(k+l+\& \mathrm{c}$.) fold locus. (It is hardly necessary to remark that such a connexion between the relations is precisely what is excluded by the foregoing definition of complete independence.) In particular if $k+l+\& c .=\omega$, the several loci may intersect, say in an $(\omega-j)$ fold locus, and besides in a residual $\omega$-fold locus, or point-system. The order (in any such case) of the residual relation or locus is equal to the product of the orders of the given relations or loci, less a reduction depending on the nature of the common relation or locus, the determination of the value of which reduction is often a complex and difficult problem.

7. Imagine a curve of given order, the equation of which contains $\omega$ arbitrary parameters: to fix the ideas, it may be assumed that these enter into the equation rationally, so that the values of the parameters being given, the curve is uniquely determined. Suppose, as above, that the parameters are taken to be the coordinates of a point in $\omega$-dimensional space; so long as the curve is not subjected to any condition, the point in question, say the parametric point, is an arbitrary point in the $\omega$-dimensional space; but if the curve be subjected to a onefold, twofold, ... or $k$-fold condition, then we have a onefold, twofold, $\ldots$ or $k$-fold relation between the parameters, and the parametric point is situate on a onefold, twofold, ... or $k$-fold locus accordingly: to each position of the parametric point on the locus there corresponds a curve satisfying the condition, that is, a solution of the condition. In the case where the condition is $\omega$-fold, the locus is a point-system, and corresponding to each point of the point-system we have a solution of the condition; the number of solutions is equal to the number of points of the point-system. 
8. Considering the general case where the condition, and therefore also the locus, is $k$-fold, it is to be observed that every solution whatever, and therefore each special solution (if any), corresponds to some point on the $k$-fold locus; we may therefore have on the $k$-fold locus what may be termed "special loci," viz. a special locus is a locus such that to each point thereof corresponds a special solution. A special locus may of course be a point-system, viz. there are in this case a determinate number of special solutions corresponding to the several points of this point-system. We may consider the other extreme case of a special $k$-fold locus, viz. the $k$-fold locus of the parametric point may break up into two distinct loci, the special $k$-fold locus, and another $k$-fold locus the several points whereot give the ordinary solutions: we can in this case get rid of the special solutions by attending exclusively to the last-mentioned $k$-fold locus and regarding it as the proper locus of the parametric point. But if the special locus be a more than $k$-fold locus, that is, if it be not a part of the $k$-fold locus itself, but (as supposed in the first instance) a locus on this locus, then the special solutions cannot be thus got rid of: we have the $k$-fold locus of the parametric point, a locus such that to every point thereof there corresponds a proper solution, save and except that to the points lying on the special locus there correspond special or improper solutions. It is to be noticed that the special locus may be, but that is not in every case, a singular locus on the $k$-fold locus.

9. Suppose that the conditions to be satisfied by the curve are a $k$-fold condition, an $l$-fold condition, \&c. of a total manifoldness $=\omega$. If the conditions are completely independent (that is, if the corresponding relations, ante, No. 5, are completely independent), we have a $k$-fold locus, an $l$-fold locus, \&c., having no common locus other than the point-system of intersection, and the number of curves which satisfy the given conditions, or (as this has been before expressed) the number of solutions, is equal to the number of points of the point-system, or to the order of the point-system, viz. it is equal to the product of the orders of the loci which correspond to the several conditions respectively; among these we may however have special solutions, corresponding to points situate on the special loci upon any of the given loci; but when this is the case the number of these special solutions can be separately calculated, and the number of proper solutions is equal to the number obtained as above, less the number of the special solutions.

10. If, however, the given conditions are not completely independent (that is, if the corresponding relations are not completely independent), then the $k$-fold locus, the $l$-fold locus, \&c. intersect in a common $(\omega-j)$ fold locus, and besides in a residual point-system. The several points of the $(\omega-j)$ fold locus give special solutions-in fact the very notion of the conditions being properly satisfied by a curve implies that the curve shall satisfy a true $(k+l+\&$ c.) fold, that is, a true $\omega$-fold condition; the proper solutions are therefore comprised among the solutions given by the residual pointsystem, and the number of them is as before equal to the order of the point-system, or number of the points thereof, less the number of points which give special solutions: the order of the point-system is, as has been seen, equal to the product of the orders of the $k$-fold locus, the $l$-fold locus, \&c., less a reduction depending on the nature of 
the common $(\omega-j)$ fold locus, and the difficulty is in general in the determination of the value of this reduction.

11. In all that precedes, the number of the parameters has been taken to be $\omega$; but if the parameters are taken to be contained in the equation of the curve homogeneously, then the parameters before made use of are in fact the ratios of these homogeneous parameters; and using the term henceforward as referring to the homogeneous parameters, the numbers of the parameters will be $=\omega+1$.

12. I assume also that the equation of the curve contains the parameters linearly : this being so, the condition that the curve shall pass through a given arbitrary point implies a linear relation between the parameters; and the condition that the curve shall pass through $j$ given points, a $j$-fold linear relation between the parameters. It follows that the number of the curves which satisfy a given $k$-fold condition, and besides pass through $\omega-k$ given points, is equal to the order of the $k$-fold relation, or of the corresponding $k$-fold locus; and thus if we define the order of the $k$-fold condition to be the number of the curves in question, the condition, relation, and locus will be all of the same order, and in all that precedes we may (in place of the order of the relation or of the locus) speak of the order of the condition. Thus, subject to the modifications occasioned by common loci and special solutions as above explained, the order of the $(k+l+\& c$.$) fold condition made up of a k$-fold condition, an $l$-fold condition, \&c., is equal to the product of the orders of the component conditions; and in particular if $k+l+\& c .=\omega$, then the order of the $\omega$-fold condition, or number of the solutions thereof, is equal to the product of the orders of the component conditions.

13. The conditions to be satisfied by the curve may be conditions of contact with a given curve or curves. In particular if the curve touch a given curve, the parametric point is then situate on a onefold locus. It is to be noticed in reference hereto that if the given curve have nodes or cusps, then we have special solutions, viz. if the sought for curve passes through a node or a cusp of the given curve; and each such node or cusp gives rise to a special onefold locus, presenting itself in the first instance as a factor of the onefold locus of the parametric point; this is, however, a case where the special locus is of the same manifoldness as the general locus (ante, No. 8), and is consequently separable; throwing off therefore all these special loci, we have a onefold locus which no longer comprises the points which correspond to curves passing through a node or a cusp of the given curve; the onefold locus, so divested of the special onefold factors, may be termed the "contact-locus" of the given curve. To each point of the contact-locus there corresponds a curve having with the given curve a two-pointic intersection, viz. this is either a proper contact, or it is a special contact, consisting in that the sought for curve has on the given curve a node or cusp, or (which is a higher speciality) in that the sought for curve is or contains as part of itself two or more coincident curves (ante, No. 3). To a point in general on the contact-locus there corresponds a curve having a proper contact with the given curve, save and except that to each point on any one of certain special loci on the contact-locus there corresponds a curve having some kind of special contact as above with the given curve. To fix the ideas, it may be mentioned that for the curves of 
the order $r$ which touch a given curve of the order $m$ and class $n$, the order of the contact-locus is $=n+(2 r-2) m$.

14. If, then, the curve touch a given curve, the parametric point is situate on the contact-locus of that curve. If it touch a second given curve, the parametric point is in like manner situate on the contact-locus of the second given curve, that is, it is situate on the twofold locus which is the intersection of the two contact-loci; and the like in the case of any number of contacts each with a distinct given curve. But if the curve, instead of ordinary contacts with distinct given curves, has either a contact of the second, or third, or any higher order, or has two or more ordinary or other contacts with the same given curve, then if the total manifoldness be $=k$, the parametric point is situate on a $k$-fold locus, which is given as a singular locus of the proper kind on the onefold contact-locus; so that the theory of the contact-locus corresponding to the case of a single contact with a given curve, contains in itself the theory of any system whatever of ordinary or other contacts with the same given curve, viz. the last-mentioned-general case depends on the discussion of the singular loci which lie on the contact-locus. And similarly, if the curve has any number of ordinary or other contacts with each of two or more given curves, we have here to consider the intersections of singular loci lying on the contact-loci which correspond to the several given curves respectively, or, what is the same thing, to the singular loci on the intersection of these contact-loci; that is, the theory depends on that of the contact-loci which belong to the given curves respectively.

15. Suppose that the curve which has to satisfy given conditions is a line; the equation is $a x+b y+c z=0$, and the parameters $(a, b, c)$ are to be taken as the coordinates of a point in a plane. Any onefold condition imposed upon the line establishes a onefold relation between the coordinates $(a, b, c)$, and the parametric point is situate on a curve; a second onefold condition imposed on the line establishes a second onefold relation between the coordinates $(a, b, c)$, and the parametric point is thus situate on a second curve; it is therefore determined as a point of intersection of two ascertained curves. In particular if the condition imposed on the line is that it shall touch a given curve, the locus of the parametric point is a curve, the contact-locus; (this is in fact the ordinary theory of geometrical reciprocity, the locus in question being the reciprocal of the given curve;) and the case of the twofold condition of a contact of the second order, or of two contacts, with the given curve, depends on the singular points of the contact-locus, or reciprocal of the given curve; in fact according as the line has a contact of the second order, or has two contacts with the given curve (that is, as it is an inflexion-tangent, or a double tangent of the given curve), the parametric point is a cusp or a node on its locus, the reciprocal curve: this is of course a funclamental notion in the theory of reciprocity, and it is only noticed here in order to show the bearing of the remark (ante, No. 14) upon the case now in hand where the curve considered is a line.

16. If the curve which has to satisfy given conditions is a conic

$$
\left(a, b, c, f, g, h \gamma(x, y, z)^{2}=0,\right.
$$

we have here six parameters $(a, b, c, f, g, h)$, which are taken as the coordinates of a 
point in 5-dimensional space. It may be remarked that in this 5 -dimensional space we have the onefold cubic locus $a b c-a f^{2}-b g^{2}-c h^{2}+2 f g h=0$, which is such that to any position of the parametric point upon it there corresponds not a proper conic but a line-pair; this may be called the discriminant-locus. We have also the threefold locus the relation of which is expressed by the six equations

$$
\left(b c-f^{2}=0, \quad c a-g^{2}=0, \quad a b-h^{2}=0, \quad g h-a f=0, \quad h f-b g=0, \quad f g-c h=0\right),
$$

which is such that to any position of the parametric point thereon, there corresponds not a proper conic but a coincident line-pair. I call this the Bipoint-locus $\left({ }^{1}\right)$, and I notice that its order is $=4$; in fact to find the order we must with the equations of the Bipoint combine two arbitrary linear relations,

$$
\begin{aligned}
& (* \gamma a, b, c, f, g, h)=0, \\
& \left(*^{\prime} \gamma a, b, c, f, g, h\right)=0 ;
\end{aligned}
$$

the equations of the locus are satisfied by

$$
a: b: c: f: g: h=\alpha^{2}: \beta^{2}: \gamma^{2}: \beta \gamma: \gamma \alpha: \alpha \beta
$$

(where $\alpha: \beta: \gamma$ are arbitrary); and substituting these values in the linear relations, we have two quadric equations in $(\alpha, \beta, \gamma)$, giving four values of the set of ratios $(\alpha: \beta: \gamma)$; that is, the order is $=4$, or the Bipoint is a threefold quadric locus.

17. The discriminant-locus does not in general present itself except in questions where it is a condition that the conic shall have a node (reduce itself to a line-pair); thus for the conics which have a node and touch a given curve $(m, n)$, or, what is the same thing, for the line-pairs which touch a given curve $(m, n)$, the parametric point is here situate on a twofold locus, the intersection of the discriminant-locus with the contact-locus. It may be noticed that this twofold locus is of the order $3(n+2 m)$, but that it breaks up into a twofold locus of the order $3 n$, which gives the proper solutions; viz. the nodal conics which touch the given curve properly, that is, one of the two lines of the conic touches the curve; and into a twice repeated twofold locus of the order $3 m$ which gives the special solutions, viz. in these the nodal conic has with the given curve a special contact, consisting in that the node or intersection of the two lines lies on the given curve. By way of illustration see Annex No. 2. But the consideration of the Bipoint-locus is more frequently necessary.

18. Suppose that the conic satisfies the condition of touching a given curve; the parametric point is then situate on a onefold contact-locus $(a, b, c, f, g, h)^{q}=0$ (to fix the ideas, if the given curve is of the order $m$ and class $n$, then the order $q$ of the contact-locus is $=n+2 m$ ). The contact-locus of any given curve whatever passes through the Bipoint-locus; in fact to each point of the Bipoint-locus there corresponds a coincident line-pair, that is, a conic which (of course in a special sense) touches the given curve whatever it be; and not only so, but inasmuch as we have a special

${ }^{1}$ In framing the epithet Bipoint, the coincident line-pair is regarded as being really a point-pair: see post, No. 30 . 
contact at each of the points of intersection of the given curve with the coincident line-pair regarded as a single line, that is, in the case of a given curve of the $m$-th order, $m$ special contacts, the Bipoint-locus is a multiple curve on the corresponding contact-locus.

19. If the conic has simply to touch a given curve of the order $m_{1}$ and class $n_{1}$, then the order of the condition (or number of the conics which satisfy the condition, and besides pass through four given points) is equal to the order of the contact-locus, that is, it is $=n_{1}+2 m_{1}$. If the conic has also to touch a second given curve of the order $m_{2}$ and class $n_{2}$, then the order of the twofold condition (or number of the conics which satisfy the twofold condition, and besides pass through three given points) is equal to the order of the intersection or common locus of the two contact-loci; and these being of the orders $n_{1}+2 m_{1}$ and $n_{2}+2 m_{2}$ respectively, the order of the intersection and therefore that of the twofold condition is $=\left(n_{1}+2 m_{1}\right)\left(n_{2}+2 m_{2}\right)$. But in the next succeeding case it becomes necessary to take account of the singular locus.

20. If the conic has to touch three given curves of the order and class $\left(m_{1}, n_{1}\right)$, $\left(m_{2}, n_{2}\right),\left(m_{3}, n_{3}\right)$ respectively, we have here three contact-loci of the orders $n_{1}+2 m_{1}$, $n_{2}+2 m_{2}, n_{3}+2 m_{3}$ respectively; these intersect in a threefold locus, but since each of the contact-loci passes through the threefold Bipoint-locus, this is part of the intersection of the three contact-loci; and not only so, but inasmuch as they pass through the Bipoint-locus $m_{1}, m_{2}, m_{3}$ times respectively, the Bipoint-locus must be counted $m_{1} m_{2} m_{3}$ times, and its order being $=4$, the intersection of the contact-locus is made up of the Bipoint reckoning as a threefold locus of the order $4 m_{1} m_{2} m_{3}$, and of a residual threefold locus of the order

$$
\begin{aligned}
& \left(n_{1}+2 m_{1}\right)\left(n_{2}+2 m_{2}\right)\left(n_{3}+2 m_{3}\right)-4 m_{1} m_{2} m_{3}, \\
= & n_{1} n_{2} n_{3}+2\left(n_{1} n_{2} m_{3}+\& \mathrm{c} .\right)+4\left(n_{1} m_{2} m_{3}+\& \mathrm{c} .\right)+4 m_{1} m_{2} m_{3} ;
\end{aligned}
$$

and the order of the threefold condition (or number of the conics which touch the three given curves, and besides pass through two given points) is equal to the order of the residual threefold locus, and has therefore the value just mentioned.

21. In going on to the cases of the conics touching four or five given curves, the same principles are applicable; the contact-loci have the Bipoint (a certain number of times repeated) as a common threefold locus, and they besides intersect in a residual fourfold or (as the case is) fivefold locus, and the order of the condition is equal to the order of this residual locus; but the determination of the order of the residual locus presents the difficulties alluded to, ante, No. 10. I do not at present further examine these cases, nor the cases of the conics which have with a given curve or curves contacts of the second or any higher order, or more than a single contact with the same given curve.

22. The equation of the conic has been in all that precedes considered as containing the six parameters $(a, b, c, f, g, h)$; but if the question as originally stated relates only to a class of conics the equation whereof contains linearly 2,3 , 4, or 5 parameters, or if, reducing the equation by means of any of the given conditions, it 
can be brought to the form in question, then in the latter case we may employ the equation in such reduced form, attending only to the remaining conditions; and in either case we have the equation of a conic containing linearly $2,3,4$, or 5 parameters, which parameters are taken as the coordinates of a point in 1-, 2-, 3-, or 4-dimensional space, and the discussion relates to loci in such dimensional space. This is in fact what is done in Annex No. 2 above referred to, where the conics considered being the conics which pass through three given points, the equation is taken to be $f y z+g z x+h x y=0$, and we have only the three parameters $(f, g, h) ;$ and also in Annex No. 3, where the conics pass through two given points, and are represented by an equation containing the four parameters $(a, b, c, h)$ : I give this Annex as a somewhat more elaborate example than any which is previously considered, of the application of the foregoing principles, and as an investigation which is interesting for its own sake. See also Annexes 4 and 5, which contain other examples of the theory. The remark as to the number of parameters is of course applicable to the case where the curve which satisfies the given conditions is a curve of any given order $r$; the number of the parameters is here at most $=\frac{1}{2}(r+1)(r+2)$, and the space therefore at most $\frac{1}{2} r(r+3)$ dimensional; but we may in particular cases have $\omega+1$ parameters, the coordinates of a point in $\omega$-dimensional space, where $\omega$ is any number less than $\frac{1}{2} r(r+3)$.

23. I do not at present consider the case of a curve of the order $r$, or further pursue these investigations; my object has been, not the development of the foregoing quasi-geometrical theory, so as to obtain thereby a series of results, but only to sketch out the general theory, and in particular to establish the notion of the order of condition, and to show that, as a rule (though as a rule subject to very frequent exceptions), the order of a compound condition is equal to the product of the orders of the component conditions. The last-mentioned theorem seems to me the true basis of the results contained in a subsequent part of this paper in connexion with the formulæ of De Jonquières, post, No. 74 et seq. But I now proceed to a different part of the general subject.

\section{Article Nos. 24 to 72.-Reproduction and Development of the Researches of Chasles and Zeuthen.}

24. The leading points of Chasles's theory are as follows: he considers the conics which satisfy four conditions $(4 X)$, and establishes the notion of the characteristics $(\mu, \nu)$ of such a system, viz. $\mu,=(4 X \cdot)$, denotes the number of conics in the system which pass through a given (arbitrary) point, and $\nu,=(4 X /)$, the number of conics in the system which touch a given (arbitrary) line. We may say that $\mu$ is the parametric order, and $\nu$ the parametric class of the system.

25. The conics

$$
(:), \quad(\therefore /), \quad(: / /), \quad(\cdot / / /), \quad(/ / / /)
$$

which pass through four given points, or which pass through three given points and touch a given line, \&c., ... or touch four given lines, have respectively the characteristics

$$
(1,2), \quad(2,4), \quad(4,4), \quad(4,2), \quad(2,1) \text {. }
$$


26. A single condition $(X)$ imposed upon a conic has two representative numbers, or simply representatives, $(\alpha, \beta)$; viz. if $(4 Z)$ be an arbitrary system of four conditions, and $(\mu, \nu)$ the characteristics of $(4 Z)$, then the number of the conics which satisfy the five conditions $(X, 4 Z)$ is $=\alpha \mu+\beta \nu$.

27. As an instance of the use of the characteristics, if $X, X^{\prime}, X^{\prime \prime}, X^{\prime \prime \prime}, X^{\prime \prime \prime \prime}$ be any five independent conditions, and $(\alpha, \beta), \ldots\left(\alpha^{\prime \prime \prime \prime}, \beta^{\prime \prime \prime \prime}\right)$ the representatives of these conditions respectively, then the number of the conics which satisfy the five conditions $\left(X, X^{\prime}, X^{\prime \prime}, X^{\prime \prime \prime}, X^{\prime \prime \prime \prime}\right)$ is

$$
=(1,2,4 ; 4,2,1 \gamma \alpha, \beta)\left(\alpha^{\prime}, \beta^{\prime}\right)\left(\alpha^{\prime \prime}, \beta^{\prime \prime}\right)\left(\alpha^{\prime \prime \prime}, \beta^{\prime \prime \prime}\right)\left(\alpha^{\prime \prime \prime \prime}, \beta^{\prime \prime \prime \prime}\right)
$$

viz. this notation stands for $1 \alpha \alpha^{\prime} \alpha^{\prime \prime} \alpha^{\prime \prime \prime} \alpha^{\prime \prime \prime \prime}+2 \Sigma \alpha \alpha^{\prime} \alpha^{\prime \prime} \alpha^{\prime \prime \prime} \beta^{\prime \prime \prime \prime} \ldots+1 \beta \beta^{\prime} \beta^{\prime \prime} \beta^{\prime \prime \prime} \beta^{\prime \prime \prime \prime}$.

28. In particular if $X$ be the condition that a conic shall touch a given curve of the order $m$ and class $n$, then the representatives of this condition are $(n, m)$, whence the number of the conics which touch each of five given curves $(m, n), \ldots$ $\left(m^{\prime \prime \prime \prime}, n^{\prime \prime \prime \prime}\right)$ is

$$
=(1,2,4,4,2,1 \gamma n, m)\left(n^{\prime}, m^{\prime}\right)\left(n^{\prime \prime}, m^{\prime \prime}\right)\left(n^{\prime \prime \prime}, m^{\prime \prime \prime}\right)\left(n^{\prime \prime \prime \prime}, m^{\prime \prime \prime \prime}\right) .
$$

29. A system of conics $(4 X)$ having the characteristics $(\mu, \nu)$, contains

$2 \nu-\mu$ line-pairs, that is, conics each of them a pair of lines; and

$2 \mu-\nu$ point-pairs, that is, conics each of them a pair of points (coniques infiniment aplaties).

30. I stop to further explain these notions of the line-pair and the point-pair; and also the notion of the line-pair-point.

A conic is a curve of the second order and second class; quid curve of the second order it may degenerate into a pair of lines, or line-pair (but the class is then $=0$ ): $q u \dot{\alpha}$ curve of the second class it may degenerate into a pair of points, or point-pair (but the order is then =0). The two lines of a line-pair may be coincident, and we have then a coincident line-pair; such a line-pair (it must I think be postulated) ordinarily arises, not from a line-pair the two lines of which become coincident, but from a proper conic, flattening by the gradual diminution of its conjugate axis, while its transverse axis remains constant or approaches a limit different from zero; the conic thus tends (not to an indefinitely extended but) to a terminated line $\left(^{1}\right.$ ); in other words, the tangents of the conic become more and more nearly lines through two fixed points, the terminations of the terminated line; and these terminating points, which continue to exist up to the instant when the conjugate axis takes its limiting value $=0$, are regarded as still existing at this instant, and the coincident line-pair as being in fact the point-pair formed by the two terminating points. Similarly the two points of a point-pair may be coincident, and we have then a coincident point-

${ }^{1}$ A line is regarded as extending from any point $A$ thereof to $B$, and then in the same direction, from $B$ through infinity to $A$; it thus consists of two portions separated by these points; and considering either portion as removed, the remaining portion is a terminated line.

C. VI. 
pair; such a point-pair (it must in like manner be postulated) ordinarily arises, not from a point-pair the two points of which become coincident, but from a proper conic sharpening itself to coincide with its asymptotes, and so becoming ultimately a pair of lines through the coincident point-pair; and the coincident point-pair is regarded as being in fact the line-pair formed by some two lines through the coincident pointpair.

31. In accordance with the foregoing notions we may with propriety, and it will in the sequel be found convenient to speak of a point-pair as a line terminated by two points on this line, and similarly to speak of a line-pair as a point terminated (that is, the pencil of lines through the point is terminated) by two lines through the point.

32. If in a point-pair thus considered as a line terminated by two points the two points become coincident (the line continuing to exist as a definite line), or, what is the same thing, if in a line-pair thus considered as a point terminated by two lines, the two lines become coincident (the point continuing to exist as a definite point), we have a "line-pair-point;" viz. this is at once a coincident line-pair and a coincident point-pair; it may also be regarded as the limit of a conic the axes of which, and the ratio of the conjugate to the transverse axis, all ultimately vanish: it may be described as a line terminated each way at a point thereof, or as a point terminated each way at a line through it. The notion of a line-pair-point first presents itself in Zeuthen's researches, as will presently appear; but it may be noticed here that line-pair-points, and these the same line-pair-points, may present themselves among the $2 \nu-\mu$ line-pairs, and among the $2 \mu-\nu$ point-pairs of the system of conics $4 X$.

33. Returning to the foregoing theory of characteristics, I remark that the fundamental notion may be taken to be, not the characteristics $(\mu, \nu)$ of the conics which satisfy four conditions, but in every case the number of the conics which satisfy five conditions. Thus for the conics not subjected to any condition, we may consider the symbols

$$
(\therefore), \quad(:: /), \quad(\therefore / /), \quad(: / / /), \quad(\cdot / / / /), \quad(/ / / / /)
$$

denoting the number of the conics which pass through five given points, or which pass through four given points and touch a given line, \&c...., or which touch five given lines; these numbers are respectively

$$
=1, \quad 2, \quad 4, \quad 4, \quad 2, \quad 1 .
$$

So for the conics which satisfy a given condition $X$, or two conditions $2 X, \ldots$, or five conditions $5 X$, we have respectively the numbers

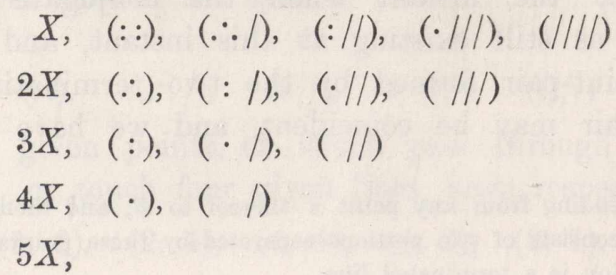


where the $X, 2 X$, \&c. belong to the symbols which follow: read $(X::),(X \therefore /)$, \&c., or, as we may for shortness represent them,

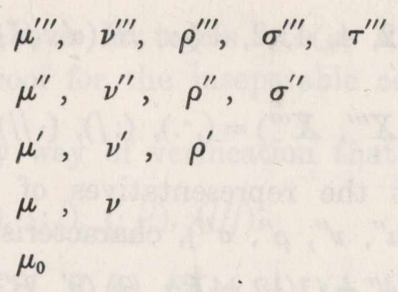

viz. the single condition $X$ has the five characteristics $\left(\mu^{\prime \prime \prime}, \ldots \tau^{\prime \prime \prime}\right), \ldots$; the four conditions $4 X$, the characteristics $(\mu, \nu)$ as in the original theory; and the five conditions $5 X$ a single characteristic $\mu_{0}$.

34. We thus see the origin of the notion of the representatives $(\alpha, \beta)$ of a single condition $X$; for considering the arbitrary four conditions $4 Z$, the characteristics whereof are $(\mu, \nu)$, and assuming that the single characteristic, or number of the conics $(X, 4 Z)$, is $=\alpha \mu+\beta \nu$, and taking for $(4 Z)$ successively the conditions

$$
(:), \quad(\therefore /), \quad(: / /), \quad(\cdot / / /), \quad(/ / / /),
$$

having respectively the characteristics

$$
(1,2), \quad(2,4), \quad(4,4), \quad(4,2), \quad(2,1),
$$

we have

$$
\begin{aligned}
& \mu^{\prime \prime \prime}=1 \alpha+2 \beta, \\
& \nu^{\prime \prime \prime}=2 \alpha+4 \beta, \\
& \rho^{\prime \prime \prime}=4 \alpha+4 \beta, \\
& \sigma^{\prime \prime \prime}=4 \alpha+2 \beta, \\
& \tau^{\prime \prime \prime}=2 \alpha+1 \beta,
\end{aligned}
$$

that is, the characteristics $\left(\mu^{\prime \prime \prime}, \nu^{\prime \prime \prime}, \rho^{\prime \prime \prime}, \sigma^{\prime \prime \prime}, \tau^{\prime \prime \prime}\right)$ of a single condition $X$ are not independent, but are representable as above by means of two independent quantities $(\alpha, \beta)$; or, what is the same thing, we have

$$
\nu^{\prime \prime \prime}=2 \mu^{\prime \prime \prime}, \quad \sigma^{\prime \prime \prime}=2 \tau^{\prime \prime \prime}, \quad \rho^{\prime \prime \prime}=\frac{2}{3}\left(\nu^{\prime \prime \prime}+\sigma^{\prime \prime \prime}\right),
$$

which being satisfied, the representatives $(\alpha, \beta)$ are given by

$$
\alpha=\frac{1}{3}\left(2 \tau^{\prime \prime \prime}-\mu^{\prime \prime \prime}\right), \quad \beta=\frac{1}{3}\left(2 \mu^{\prime \prime \prime}-\tau^{\prime \prime \prime}\right) .
$$

35. I find that a like property exists as to the characteristics $\left(\mu^{\prime \prime}, \nu^{\prime \prime}, \rho^{\prime \prime}, \sigma^{\prime \prime}\right)$ of the two conditions $2 X$, viz. these are not independent but are connected by a single linear relation,

$$
\mu^{\prime \prime}-\frac{3}{2} \nu^{\prime \prime}+\frac{3}{2} \rho^{\prime \prime}-\sigma^{\prime \prime}=0 .
$$

This may be proved in the case where the conditions $2 X$ are two separate conditions $\left(X, X^{\prime}\right)$; viz. let the representatives of these be $(\alpha, \beta),\left(\alpha^{\prime}, \beta^{\prime}\right)$ respectively, then 
combining with them the three arbitrary conditions $X^{\prime \prime}, X^{\prime \prime \prime}, X^{\prime \prime \prime}$ having respectively the representatives $\left(\alpha^{\prime \prime}, \beta^{\prime \prime}\right),\left(\alpha^{\prime \prime \prime}, \beta^{\prime \prime \prime}\right),\left(\alpha^{\prime \prime \prime \prime}, \beta^{\prime \prime \prime \prime}\right)$, we have the general equation

$$
\left(X, X^{\prime}, X^{\prime \prime}, X^{\prime \prime \prime}, X^{\prime \prime \prime \prime}\right)=\left(1,2,4,4,2,1 \gamma(\alpha, \beta)\left(\alpha^{\prime}, \beta^{\prime}\right)\left(\alpha^{\prime \prime}, \beta^{\prime \prime}\right)\left(\alpha^{\prime \prime \prime}, \beta^{\prime \prime \prime}\right)\left(\alpha^{\prime \prime \prime \prime}, \beta^{\prime \prime \prime \prime}\right)\right. \text {; }
$$

taking herein

$$
\left(X^{\prime \prime}, X^{\prime \prime \prime}, X^{\prime \prime \prime}\right)=(\therefore),(: /),(\cdot / /),(/ / /)
$$

successively, and observing that the representatives of $(\cdot)$ are $(1,0)$ and those of $(/)$ are $(0,1)$, we thus obtain for $\left(\mu^{\prime \prime}, \nu^{\prime \prime}, \rho^{\prime \prime}, \sigma^{\prime \prime}\right)$, characteristics of $\left(X, X^{\prime}\right)$, the values

$$
\begin{aligned}
& \mu^{\prime \prime}=(1,2,4 \gamma \alpha, \beta)\left(\alpha^{\prime}, \beta^{\prime}\right), \\
& \nu^{\prime \prime}=(2,4,4 \gamma \alpha, \beta)\left(\alpha^{\prime}, \beta^{\prime}\right), \\
& \rho^{\prime \prime}=(4,4,2 \gamma \alpha, \beta)\left(\alpha^{\prime}, \beta^{\prime}\right), \\
& \sigma^{\prime \prime}=(4,2,1 \gamma \alpha, \beta)\left(\alpha^{\prime}, \beta^{\prime}\right),
\end{aligned}
$$

(viz. $\mu^{\prime \prime}=1 \alpha \alpha^{\prime}+2\left(\alpha \beta^{\prime}+\alpha^{\prime} \beta\right)+4 \beta \beta^{\prime}$, \&c.), and these values give identically

$$
2 \mu^{\prime \prime}-3 \nu^{\prime \prime}+3 \rho^{\prime \prime}-2 \sigma^{\prime \prime}=0 \text {, }
$$

which is the foregoing equation. And I assume that the theorem extends to the case of two inseparable conditions $2 X$, but in this case $I$ do not even know where the proof is to be sought for.

The characteristics $\left(\mu^{\prime}, \nu^{\prime}, \rho^{\prime}\right)$ of the three conditions $3 X$ are in general independent.

36. It has been mentioned that if $(\alpha, \beta)$ are the representatives of the condition $X$, and $(\mu, \nu)$ the characteristics of the conditions $4 Z$, then

$$
(X, 4 Z)=\alpha \mu+\beta \nu
$$

this is the most convenient form of the theorem, but as $(\alpha, \beta)$ are known functions of the characteristics $\left(\mu^{\prime \prime \prime}, \nu^{\prime \prime \prime}, \rho^{\prime \prime \prime}, \sigma^{\prime \prime \prime}, \tau^{\prime \prime \prime}\right)$ of the condition $X$, the equation is in effect an expression for $(X, 4 Z)$ in terms of the charaeteristics of $X$ and $4 Z$ respectively.

There is, similarly, an expression for $(2 X, 3 Z)$ in terms of the characteristics $\left(\mu^{\prime}, \nu^{\prime}, \rho^{\prime}, \sigma^{\prime}\right)$ of $3 Z$ (satisfying the relation $\left.\mu^{\prime}-\frac{3}{2} \nu^{\prime}+\frac{3}{2} \rho^{\prime}-\sigma^{\prime}=0\right)$ and the characteristics $(\mu, \nu, \rho)$ of $2 X$, viz. we have

$$
\begin{aligned}
(2 X, 3 Z)= & \mu\left(-\frac{1}{4} \rho^{\prime}+\frac{1}{2} \sigma^{\prime}\right) \\
& +\nu\left(-\frac{3}{8} \mu^{\prime}+\frac{5}{16} \nu^{\prime}+\frac{5}{16} \rho^{\prime}-\frac{3}{8} \sigma^{\prime}\right) \\
& +\rho\left(\frac{1}{2} \mu^{\prime}-\frac{1}{4} \nu^{\prime}\right.
\end{aligned}
$$

This may be easily proved in the case where the conditions $2 X$ are two separable conditions $X, X^{\prime}$ having the representatives $(\alpha, \beta),\left(\alpha^{\prime}, \beta^{\prime}\right)$ respectively, and the conditions $3 Z$ three separable conditions $Z^{\prime \prime}, Z^{\prime \prime \prime}, Z^{\prime \prime \prime}$ having the representatives $\left(\alpha^{\prime \prime}, \beta^{\prime \prime}\right)$, $\left(\alpha^{\prime \prime \prime}, \beta^{\prime \prime \prime}\right),\left(\alpha^{\prime \prime \prime \prime}, \beta^{\prime \prime \prime \prime}\right)$ respectively; we have, in fact,

$$
\begin{aligned}
& \mu^{\prime}=\left(1,2,4 \gamma(\alpha, \beta)\left(\alpha^{\prime}, \beta^{\prime}\right), \quad \mu=\left(1,2,4,4 \gamma\left(\alpha^{\prime \prime}, \beta^{\prime \prime}\right)\left(\alpha^{\prime \prime \prime}, \beta^{\prime \prime \prime}\right)\left(\alpha^{\prime \prime \prime \prime}, \beta^{\prime \prime \prime \prime}\right)\right. \text {, }\right.
\end{aligned}
$$

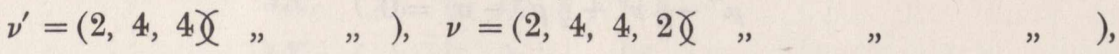

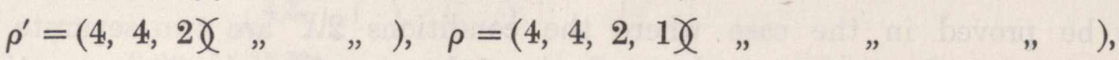

$$
\begin{aligned}
& \sigma^{\prime}=(4,2,1 \chi \ldots,) \text {; }
\end{aligned}
$$


and with these values the function

$$
\left(X, X^{\prime}, Z^{\prime \prime}, Z^{\prime \prime \prime}, Z^{\prime \prime \prime \prime}\right),=(1,2,4,4,2,1 \gamma \alpha, \beta)\left(\alpha^{\prime}, \beta^{\prime}\right)\left(\alpha^{\prime \prime}, \beta^{\prime \prime}\right)\left(\alpha^{\prime \prime \prime}, \beta^{\prime \prime \prime}\right),\left(\alpha^{\prime \prime \prime \prime}, \beta^{\prime \prime \prime \prime}\right)
$$

is found to be expressible as above in terms of $(\mu, \nu, \rho),\left(\mu^{\prime}, \nu^{\prime}, \rho^{\prime}, \sigma^{\prime}\right)$; but I do not know how to conduct the proof for the inseparable conditions $2 X$ and $3 Z$.

37. It may be remarked by way of verification that writing successively

that is,

$$
(3 Z)=(\therefore),(: /),(\cdot / /),(/ / /),
$$

$$
(\mu, \nu, \rho)=(1,2,4),(2,4,4),(4,4,2),(4,4,1),
$$

we have in the first case

$$
\begin{aligned}
(2 X \therefore)= & -\frac{1}{4} \rho^{\prime}+\frac{1}{2} \sigma^{\prime} \\
& -\frac{3}{4} \mu^{\prime}+\frac{5}{8} \nu^{\prime}+\frac{5}{8} \rho^{\prime}-\frac{3}{4} \sigma^{\prime} \\
& +2 \mu^{\prime}-\nu^{\prime} \\
= & \mu^{\prime}+\frac{1}{4}\left(\mu^{\prime}-\frac{3}{2} \nu^{\prime}+\frac{3}{2} \rho^{\prime}-\sigma^{\prime}\right),=\mu^{\prime},
\end{aligned}
$$

and similarly in the other three cases,

$$
(2 X \cdot / /)=\nu^{\prime}, \quad(2 X \cdot / /)=\rho^{\prime}, \quad(2 X / / /)=\sigma^{\prime} .
$$

38. Let $(\mu, \nu, \rho, \sigma)$ be the characteristics of $2 Z,\left(\mu-\frac{3}{2} \nu+\frac{3}{2} \rho-\sigma=0\right)$, and $\left(\mu^{\prime}, \nu^{\prime}, \rho^{\prime}, \sigma^{\prime}\right)$ the characteristics of $2 X,\left(\mu^{\prime}-\frac{3}{2} \nu^{\prime}+\frac{3}{2} \rho^{\prime}-\sigma^{\prime}=0\right)$. Then in the formula for $(2 X, 3 Z)$, writing successively for $3 X$

and

$$
(2 X \cdot) \text {, characteristics }(\mu, \nu, \rho) \text {, }
$$

$$
(2 X /), \quad \# \quad(\nu, \rho, \sigma),
$$

we obtain expressions for the characteristics $(2 X, 2 Z \cdot)$ and $(2 X, 2 Z /)$ of $(2 X, 2 Z)$, viz. eliminating from the formulæ, first the $\left(\sigma, \sigma^{\prime}\right)$ and secondly the $\left(\mu, \mu^{\prime}\right)$, each of these may be expressed in two different forms as follows:

$$
\begin{aligned}
& (2 X, 2 Z \cdot) \\
= & \frac{1}{2} \mu \mu^{\prime} \\
+ & \frac{7}{8} \nu \nu^{\prime} \\
- & \frac{3}{4}\left(\mu \nu^{\prime}+\mu^{\prime} \nu\right) \\
+ & \frac{1}{2}\left(\mu \rho^{\prime}+\mu^{\prime} \rho\right) \\
- & \frac{1}{4}\left(\nu \rho^{\prime}+\nu^{\prime} \rho\right) \\
= & \frac{1}{2} \sigma \sigma^{\prime} \\
- & \frac{3}{8} \rho \rho^{\prime} \\
- & \frac{1}{4} \nu \nu^{\prime} \\
- & \frac{1}{4}\left(\rho \sigma^{\prime}+\rho^{\prime} \sigma\right) \\
+ & \frac{1}{2}\left(\nu \rho^{\prime}+\nu^{\prime} \rho\right),
\end{aligned}
$$

$$
\begin{aligned}
& (2 X, 2 Z /) \\
= & \frac{1}{2} \mu \mu^{\prime} \\
- & \frac{3}{8} \nu \nu^{\prime} \\
- & \frac{1}{4} \rho \rho^{\prime} \\
- & \frac{1}{4}\left(\mu \nu^{\prime}+\mu^{\prime} \nu\right) \\
+ & \frac{1}{2}\left(\nu \rho^{\prime}+\nu^{\prime} \rho\right) \\
= & \frac{1}{2} \sigma \sigma^{\prime} \\
+ & \frac{7}{8} \rho \rho^{\prime} \\
- & \frac{3}{4}\left(\rho \sigma^{\prime}+\rho^{\prime} \sigma\right) \\
+ & \frac{1}{2}\left(\nu \sigma^{\prime}+\nu^{\prime} \sigma\right) \\
- & -\frac{1}{4}\left(\nu \rho^{\prime}+\nu^{\prime} \rho\right),
\end{aligned}
$$


the two expressions of the same quantity being of course equivalent in virtue of the relations between $(\mu, \nu, \rho, \sigma)$ and $\left(\mu^{\prime}, \nu^{\prime}, \rho^{\prime}, \sigma^{\prime}\right)$ respectively.

The characteristics of $(X, Z),(X, 2 Z),(X, 3 Z)$ are at once deducible from the before-mentioned expression $\alpha \mu+\beta \nu$ of $(X, 4 Z)$.

39. Zeuthen's investigations are based upon the before-mentioned theorem, that in a system of conics $(4 X)$, characteristics $(\mu, \nu)$, there are $2 \mu-\nu$ point-pairs and $2 \nu-\mu$ line-pairs. If in the given system the number of point-pairs is $=\lambda$ and the number of line-pairs is $=\sigma$, then, conversely, the characteristics of the system are

$$
\mu=\frac{1}{3}(2 \lambda+\varpi), \quad \nu=\frac{1}{3}(\lambda+2 \varpi) .
$$

And by means of this formula he investigates the characteristics of the several systems of conics which satisfy four conditions ( $4 X)$ of contact with a given curve or curves, viz. these are the conics
(1) (1) (1) (1),
$(1,1)(1)(1)$,
$(1,1)(1,1)$,
$(1,1,1)(1)$,
$(1,1,1,1)$
(2) (1) (1)
(2) $(1,1)$
$(2,1)(1)$,
$(2,1,1)$,

where (1) denotes contact of the first order, (2) of the second order, (3) of the third order, (4) of the fourth order, with a given curve; (1)(1) denotes contacts of the first order with each of two given curves, $(1,1)$ two such contacts with the same given curve, and so on. A given curve is in every case taken to be of the order $m$ and class $n$, with $\delta$ nodes, $\kappa$ cusps, $\tau$ double tangents, and $\iota$ inflexions $\left(m_{1}, n_{1}, \delta_{1}, \kappa_{1}, \tau_{1}, \iota_{1}\right.$; $m_{2}, n_{2}$, \&c., as the case may be). The symbols (1), \&c. might be referred to the corresponding curves by a suffix; thus $(1)_{m}$ would denote that the contact is with a given curve of the order $m$ (class $n$, \&c.); but this is in general unnecessary.

40. In a system of conics satisfying four conditions of contact, as above, it is comparatively easy to see what are the point-pairs and line-pairs in these several systems respectively; but in order to find the values of $\lambda$ and $\varpi$, each of these pointpairs and line-pairs has to be counted not once, but a proper number of times; and it is in the determination of these multiplicities that the difficulty of the problem consists. I do not enter into this question, but give merely the results.

41. For the statement of these I introduce what I call the notation of Zeuthen's Capitals. We have to consider several classes of point-pairs and the reciprocal classes of line-pairs. A point-pair may be described (ante, No. 31) as a terminated line, and a line-pair as a terminated point; and we have first the following point-pairs, viz.:

$A$, line terminated each way in the intersection of two curves or of a curve with itself (node).

$B$, tangent to a curve, terminated in a curve, and in the intersection of two curves or of a curve with itself. 
$C$, common tangent of two curves, or double tangent of a curve, terminated each way in a curve.

$D$, inflexion tangent of a curve terminated each way in a curve :

and the corresponding line-pairs, viz. :

$A^{\prime}$, point terminated each way in the common tangent of two curves or the double tangent of a curve.

$B^{\prime}$, point of a curve terminated by the tangent of a curve, and by the common tangent of two curves or double tangent of a curve.

$C^{\prime}$, intersection of two curves, or of a curve with itself (node), terminated each way by the tangent to a curve.

$D^{\prime}$, cusp of a curve terminated each way by the tangent to a curve :

all which is further explained, by what follows; thus in the case (1)(1)(1) (1), $=(1)_{m_{1}}(1)_{m_{2}}(1)_{m_{3}}(1)_{m_{4}}$, the value of $A$ is given as $\Sigma m_{1} m_{2} \cdot m_{3} m_{4}\left(=3 m_{1} m_{2} m_{3} m_{4}\right)$. Here $A$ is the number of the point-pairs terminated one way in the intersection of any two $m_{1}, m_{2}$ of the four curves, and the other way in the intersection of the remaining two $m_{3}, m_{4}$ of the four curves. But in the case $(1,1)(1)(1),=(1,1)_{m}(1)_{m_{1}}(1)_{m_{2}}$, the value of $A$ is given as $=\delta m_{1} m_{2}+m m_{1} \cdot m m_{2}$. Here $A$ denotes the number of the point-pairs, which are either $\left(\delta m_{1} m_{2}\right)$ terminated one way at a node of $m$, and the other way at an intersection of $m_{1}, m_{2}$, or else $\left(m m_{1}, m m_{2}\right)$ terminated one way at an intersection of $m, m_{1}$, and the other way at an intersection of $m, m_{2}$ : and so in other cases.

42. This being so, we have

(1) $(1)(1)(1),=(1)_{m_{1}}(1)_{m_{2}}(1)_{m_{3}}(1)_{m_{4}}$.

\begin{tabular}{ll|l|ll}
$A=\Sigma m_{1} m_{2}$ & $\cdot m_{3} m_{4}\left(=3 \quad m_{1} m_{2} m_{3} m_{4}\right)$, & 1 & $A^{\prime}=\Sigma n_{1} n_{2} \cdot n_{3} n_{4} \quad\left(=3 n_{1} n_{2} n_{3} n_{4}\right)$, \\
$B=\Sigma m_{1} m_{2} \cdot m_{3} \cdot n_{4}\left(=3 \Sigma m_{1} m_{2} m_{3} n_{4}\right)$, & 2 & $B^{\prime}=\Sigma n_{1} n_{2} \cdot n_{3} \cdot m_{4}\left(=3 \Sigma n_{1} n_{2} n_{3} m_{4}\right)$, \\
$C=\Sigma m_{1} \cdot m_{2} \cdot n_{3} n_{4} \quad\left(=\Sigma m_{1} m_{2} n_{3} n_{4}\right)$. & 4 & $C^{\prime}=\Sigma n_{1} \cdot n_{2} \cdot m_{3} m_{4} \quad\left(=\Sigma n_{1} n_{2} m_{3} m_{4}\right)$.
\end{tabular}

$(1,1)(1)(1),=(1,1)_{m}(1)_{m_{1}}(1)_{m_{2}}$.

$$
\begin{aligned}
A= & \delta m_{1} m_{2} \quad+m m_{1} \cdot m m_{2}, \\
B= & \delta n_{1} m_{2} \quad+\delta n_{2} m_{1} \\
& +m m_{1}(n-2) m_{2}+m m_{2}(n-2) m_{1} \\
& +m m_{1} n_{2}(m-1)+m m_{2} n_{1}(m-1) \\
& +m_{1} m_{2} n(m-2) \\
C= & \tau m_{1} m_{2} \\
& +n n_{1}(m-2) m_{2}+n n_{2}(m-2) m_{1} \\
& +n_{1} n_{2} \cdot \frac{1}{2} m(m-1), \\
D= & \iota m_{1} m_{2} .
\end{aligned}
$$




$(1,1)(1,1),=(1,1)_{m}(1,1)_{m_{1}}$.

$$
\begin{aligned}
& A=\delta \delta_{1} \\
& +\frac{1}{2} m m_{1}\left(m m_{1}-1\right) \text {, } \\
& B=\delta n_{1}\left(m_{1}-2\right) \\
& +\delta_{1} n(m-2) \\
& +m m_{1}(n-2)\left(m_{1}-1\right)+m m_{1}\left(n_{1}-2\right)(m-1) \text {, } \\
& C=\tau \cdot \frac{1}{2} m_{1}\left(m_{1}-1\right)+\tau_{1} \cdot \frac{1}{2} m(m-1) \\
& +n n_{1}(m-2)\left(m_{1}-2\right) \text {, } \\
& D=\iota \cdot \frac{1}{2} m_{1}\left(m_{1}-1\right) \\
& +\iota_{1} \cdot \frac{1}{2} m(m-1) .
\end{aligned}
$$



$(1,1,1)(1)=(1,1,1)_{m}(1)_{m_{1}}$.

$$
\begin{array}{rl|l|l}
A=\delta m m_{1}, & 1 & A^{\prime}=\tau n n_{1}, \\
B= & \delta(n-4) m_{1}+\delta n_{1}(m-2), & 2 & B^{\prime}=\tau(m-4) n_{1}+\tau m_{1}(n-2), \\
& +m m_{1}(n-2)(m-3), & & +n n_{1}(m-2)(n-3), \\
C= & & 4(m-4) m_{1}+n n_{1} \cdot \frac{1}{2}(m-2)(m-3), & C^{\prime}=\delta(n-4) n_{1}+m m_{1} \cdot \frac{1}{2}(n-2)(n-3), \\
D= & \iota(m-3) m_{1} . & 3 & D^{\prime}=\kappa(n-3) n_{1} .
\end{array}
$$

$(1,1,1,1),=(1,1,1,1)_{m}$.

$$
\begin{aligned}
& A=\frac{1}{2} \delta(\delta-1), \\
& B=\delta(n-4)(m-4), \\
& C=\tau \cdot \frac{1}{2}(m-4)(m-5), \\
& D=\iota \cdot \frac{1}{2}(m-3)(m-4) .
\end{aligned}
$$

$$
\begin{array}{l|l}
1 & A^{\prime}=\frac{1}{2} \tau(\tau-1) \\
2 & B^{\prime}=\tau(m-4)(n-4) \\
3 & C^{\prime}=\delta \cdot \frac{1}{2}(n-4)(n-5) \\
4 & D^{\prime}=\kappa \cdot \frac{1}{4}(n-3)(n-4)
\end{array}
$$

43. Secondly, we have the point-pairs:

$E$, tangent to curve from intersection of two curves or of a curve with itself (node), and terminated at the point of contact and the last-mentioned point.

$F$, tangent to a curve at intersection with another curve or with itself, and terminated there and at a curve.

$G$, common tangent of two curves or double tangent of a curve, terminated at one of the points of contact and at a curve.

D, ut suprà.

$H$, line joining cusp of a curve with intersection of two curves or of a curve with itself, and terminated at these points.

$I$, line from cusp of a curve touching a curve, and terminated at the cusp and at a curve.

$J$, Inflexion tangent of a curve, terminated there and at a curve: 
and the corresponding line-pairs, viz.

$E^{\prime}$, point on a curve in common tangent of two curves or double tangent of a curve, and terminated by this tangent and by tangent to a curve.

$F^{\prime}$, point on a curve in common tangent of this and another curve or in double tangent of this curve, and terminated by this tangent and by tangent to a curve.

$D^{\prime}$, ut suprà.

$H^{\prime}$, intersection of inflexion tangent of a curve with common tangent of two curves or double tangent of a curve, and terminated by these lines.

$I^{\prime}$, intersection of inflexion tangent of a curve with a curve, and terminated by this tangent and by tangent of a curve:

and this being so,

(2) (1) (1), = (2) $)_{m}(1)_{m_{1}}(1)_{m_{2}}$.

$$
\begin{aligned}
& E=n \cdot m_{1} m_{2}, \\
& F=m m_{1} \cdot m_{2}+m m_{2} \cdot m_{1}, \\
& G=n n_{2} \cdot m_{1}+n n_{1} \cdot m_{2}, \\
& D=\iota m_{1} m_{2}, \\
& H=\kappa m_{1} m_{2}, \\
& I=\kappa n_{1} m_{2} \quad+\kappa n_{2} m_{1} .
\end{aligned}
$$

\begin{tabular}{l|l}
3 & $E^{\prime}=m \cdot n_{1} n_{2}$, \\
3 & $F^{\prime}=n n_{1} \cdot m_{2}+n n_{2} \cdot m_{1}$, \\
6 & $G^{\prime}=m m_{2} \cdot n_{1}+m n_{1} \cdot n_{2}$, \\
2 & $D^{\prime}=\kappa n_{1} n_{2}$, \\
1 & $H^{\prime}=\iota n_{1} n_{2}$, \\
2 & $I^{\prime}=\iota m_{2} n_{1} \quad+\iota m_{2} n_{1}$.
\end{tabular}

$(2)(1,1)=(2)_{m}(1,1)_{m_{1}}$.

$$
\begin{aligned}
& E=\delta_{1} n, \\
& F=m \cdot m_{1}\left(m_{1}-1\right), \\
& G=n n_{1} \quad\left(m_{1}-2\right), \\
& D=\iota \cdot \frac{1}{2} m_{1}\left(m_{1}-1\right), \\
& H=\kappa \delta_{1}, \\
& I=\kappa n_{1} \quad\left(m_{1}-2\right) .
\end{aligned}
$$

\begin{tabular}{l|l}
3 & $E^{\prime}=\tau_{1} m$, \\
3 & $F^{\prime}=n \cdot n_{1} \quad\left(n_{1}-1\right)$, \\
6 & $G^{\prime}=m m_{1} \quad\left(n_{1}-2\right)$, \\
2 & $D^{\prime}=\kappa \cdot \frac{1}{2} n_{1}\left(n_{1}-1\right)$, \\
1 & $H^{\prime}=\iota \tau_{1}$ \\
2 & $I^{\prime}=\iota m_{1} \quad\left(n_{1}-2\right)$,
\end{tabular}

$$
(2,1)(1),=(2,1)_{m}(1)_{m_{1}} \text {. }
$$

$$
\begin{aligned}
& E=(n-2) \cdot m m_{1}, \\
& F=m m_{1}(m-2)+2 \delta m_{1}, \\
& G=n n_{1}(m-2)+2 \tau m_{1}, \\
& D=\iota(m-3) m_{1}, \\
& H=\kappa m m_{1}, \\
& I=\kappa(n-3) m_{1}+\kappa n_{1}(m-2), \\
& J=\iota m_{1} .
\end{aligned}
$$$$
\begin{array}{l|l}
3 & E^{\prime}=(m-2) \cdot n n_{1}, \\
3 & F^{\prime}=n n_{1}(n-2)+2 \tau n_{1}, \\
6 & G^{\prime}=m m_{1}(n-2)+2 \delta n_{1}, \\
2 & D^{\prime}=\kappa(n-3) n_{1}, \\
1 & H^{\prime}=\iota n n_{1}, \\
2 & I^{\prime}=\iota(m-3) n_{1}+\iota m_{1}(n-2), \\
5 & J^{\prime}=\kappa n_{1} .
\end{array}
$$

C. VI. 
$(2,1,1),=(2,1,1)_{m}$.

$$
\begin{array}{l|l|l}
E=\delta(n-4), & 3 & E^{\prime}=\tau(m-4), \\
F=2 \delta(m-3), & 3 & F^{\prime}=2 \tau(n-3), \\
G=2 \tau(m-4), & 6 & G^{\prime}=2 \delta(n-4), \\
D=\iota \cdot \frac{1}{2}(m-3)(m-4), & 2 & D^{\prime}=\kappa \cdot \frac{1}{2}(n-3)(n-4), \\
H=\delta \kappa, & 1 & H^{\prime}=\iota \tau, \\
I=\kappa(n-3)(m-4), & 2 & I^{\prime}=\iota(m-3)(n-4), \\
J=\iota(m-3) . & 5 & J^{\prime}=\kappa(n-3) .
\end{array}
$$

44. Thirdly, we have the point-pairs:

$K$, common tangent of two curves or double tangent of a curve, terminated at points of contact.

$L$, line from cusp of a curve touching a curve, and terminated at cusp and point of contact.

$M$, line joining cusp of a curve with cusp of a curve, and terminated by the two cusps.

$N$, inflexion tangent terminated each way at inflexion, viz. this is a line-pair-point.

0 , cuspidal tangent terminated each way at cusp, viz. this is a line-pair-point:

and the corresponding line-pairs :

$K^{\prime}$, intersection of two curves or of curve with itself (node), and terminated by the two tangents.

$L^{\prime}$, intersection of inflexion tangent of a curve with a curve, and terminated by the inflexion tangent and the tangent at the intersection.

$M^{\prime}$, intersection of inflexion tangent of a curve with inflexion tangent of a curve, and terminated by the two inflexion tangents.

$N^{\prime},=0$, line-pair-point as above.

$O^{\prime},=N$, line-pair-point as above:

which being so, we have

$$
\begin{aligned}
& \text { (2) }(2),=(2)_{m}(2)_{m_{1}} \text {. }
\end{aligned}
$$




$(2,2),=(2,2)_{m}$.

$$
\begin{array}{l|l|l}
K=\tau, & 9 & K^{\prime}=\delta, \\
L=\kappa(n-3), & 3 & L^{\prime}=\iota(m-3), \\
M=\frac{1}{2} \kappa(\kappa-1), & 1 & M^{\prime}=\frac{1}{2} \iota(\iota-1), \\
N=\iota, & 2 & N^{\prime}=\kappa, \\
O=\kappa . & O^{\prime}=\iota .
\end{array}
$$

45. Fourthly, we have the point-pairs:

$P$, tangent of a curve at its intersection with another curve or itself, terminated each way at the point of contact-line-pair-point.

Q, common tangent of two curves or double tangent of a curve, terminated each way at one of the points of contact-line-pair-point.

$J$, ut suprà.

$R$, cuspidal tangent terminated at cusp and at a curve:

and the corresponding line-pairs:

$P^{\prime},=Q$, line-pair-point

$Q^{\prime},=P$, line-pair-point.

$J^{\prime}$, ut suprà.

$R^{\prime}$, inflexion of curve terminated by the inflexion tangent and by tangent to a curve :

which being so, we have

(3) $(1),=(3)_{m}(1)_{m_{1}}$.

$$
\begin{aligned}
& P=m m_{1} \text {, } \\
& Q=n n_{1} \text {, } \\
& J=\iota m_{1} \text {, } \\
& R=\kappa m_{1} \text {. } \\
& P^{\prime}=n n_{1}, \\
& Q^{\prime}=m m_{1} \text {, } \\
& J^{\prime}=\kappa n_{1} \text {, } \\
& D^{\prime}=\imath n_{1} \text {. }
\end{aligned}
$$

$\left|\begin{array}{l}2 \\ 2 \\ 5 \\ 4\end{array}\right|$

$(3,1),=(3,1)_{m}$.

$$
\begin{aligned}
& P=2 \delta, \\
& Q=2 \tau, \\
& J=\iota(m-3), \\
& R=\kappa(m-3) .
\end{aligned}
$$

\begin{tabular}{l|l}
2 \\
2 \\
5
\end{tabular} \mid $\begin{aligned} & Q^{\prime}=2 \tau, \\
& 4\end{aligned} \quad \begin{aligned} & P^{\prime}=2 \delta, \\
& J^{\prime}=\kappa(n-3), \\
& R^{\prime}=\iota(n-3) .\end{aligned}$

46. And lastly, we have the point-pairs $N, O$ (line-pair-points) and the line-pairs $N^{\prime}, O^{\prime}$ (line-pair-points), ut suprò, and

$$
\begin{aligned}
(4),=(4)_{m} . \\
N=\iota, \\
O=\kappa .
\end{aligned}
$$$$
\begin{array}{l|l}
4 & N^{\prime}=\kappa, \\
2 & O^{\prime}=\iota .
\end{array}
$$ 
47. Where in all cases the central column of figures gives the numerical factors which multiply the corresponding capitals, thus we have

for $(1)(1)(1)(1)$

for $(1,1)(1)(1)$,

$$
\begin{aligned}
& \lambda=2 \nu-\mu=A+2 B+4 C, \\
& \varpi=2 \mu-\nu=A^{\prime}+2 B^{\prime}+4 C^{\prime} ;
\end{aligned}
$$

and so on.

$$
\begin{aligned}
& \lambda=2 \nu-\mu=A+2 B+4 C+3 D, \\
& \varpi=2 \mu-\nu=A^{\prime}+2 B^{\prime}+4 C^{\prime}+3 D^{\prime},
\end{aligned}
$$

48. The elements $(m, n, \delta, \kappa, \tau, \iota)$ of a curve satisfy Plücker's six equations, and Zeuthen uses these equations, in a somewhat unsystematic way, to simplify the form of his results.

It is convenient in his formulæ to write $3 m+\iota,=3 n+\kappa,=\alpha$, and to express everything in terms of $(m, n, \alpha)$, viz. we have for this purpose

$$
\begin{aligned}
& 2 \delta=m^{2}-m+8 n-3 \alpha, \\
& 2 \tau=n^{2}-8 m-n-3 \alpha .
\end{aligned}
$$

But I make another alteration in the form of his results; he gives, for instance, the characteristics of $(1,1)(1)(1)$ as

where

$$
\begin{aligned}
& \mu=\mu^{\prime \prime \prime} m_{1} m_{2}+\mu^{\prime \prime}\left(m_{1} n_{2}+m_{2} n_{1}\right)+\mu^{\prime} n_{1} n_{2}, \\
& \nu=\nu^{\prime \prime \prime} m_{1} m_{2}+\nu^{\prime \prime}\left(m_{1} n_{2}+m_{2} n_{1}\right)+\nu^{\prime} n_{1} n_{2},
\end{aligned}
$$

$$
\begin{aligned}
\mu^{\prime} & =2 m(m+n-3)+\tau,=(1,1 \therefore), \\
\mu^{\prime \prime}=\nu^{\prime} & =2 n(m+2 n-5)+2 \tau,=(1,1: /), \\
\mu^{\prime \prime \prime}=\nu^{\prime \prime} & =2 n(2 m+n-5)+2 \delta,=(1,1 \cdot / /), \\
\nu^{\prime \prime \prime} & =2 n(m+n-3)+\delta,=(1,1 / / /),
\end{aligned}
$$

viz. the four components have really the significations $(1,1 \therefore)$ set opposite to them respectively; and accordingly, instead of giving the formulæ for the two characteristics of $(1,1)(1)(1)$, I give those for the four characteristics $(1,1.2)$, \&c. of $(1,1)$, thus in every case obtaining formulæ which relate to a single curve only. Subject to the lastmentioned variation of form, I give Zeuthen's original expressions in Annex 6; but here in the text $\mathbf{I}$ express them as above in terms of $(m, n, \alpha)$, viz.

49. We have the formulæ

(1)

$$
\begin{aligned}
& (::)=n+2 m, \\
& (\therefore /)=2 n+4 m, \\
& (: / /)=4 n+4 m, \\
& (\cdot / / /)=4 n+2 m, \\
& (/ / / /)=2 n+2 m ;
\end{aligned}
$$


$(1,1)$

$$
\begin{aligned}
& (\therefore)=2 m^{2}+2 m n+\frac{1}{2} n^{2}-2 m-\frac{1}{2} n-\frac{3}{2} \alpha, \\
& (: /)=2 m^{2}+4 m n+n^{2}-2 m-n-3 \alpha, \\
& (\cdot / /)=m^{2}+4 m n+2 n^{2}-m-2 n-3 \alpha, \\
& (/ / /)=\frac{1}{2} m^{2}+2 m n+2 n^{2}-\frac{1}{2} m-2 n-\frac{3}{2} \alpha .
\end{aligned}
$$

$(1,1,1)$

(:) $=\frac{2}{3} m^{3}+2 m^{2} n+m n^{2}+\frac{1}{6} n^{3}-2 m^{2}-3 m n-\frac{1}{2} n^{2}-\frac{20}{3} m-\frac{29}{3} n+\alpha\left(-3 m-\frac{3}{2} n+13\right)$,

$(\cdot /)=\frac{1}{3} m^{3}+2 m^{2} n+2 m n^{2}+\frac{1}{3} n^{3}-m^{2}-4 m n-n^{2}-\frac{46}{3} m-\frac{46}{3} n+\alpha(-3 m-3 n+20)$,

$(/ /)=\frac{1}{6} m^{3}+m^{2} n+2 m n^{2}+\frac{2}{3} n^{3}-\frac{1}{2} m^{2}-3 m n-2 n^{2}-\frac{29}{3} m-\frac{20}{3} n+\alpha\left(-\frac{3}{2} m-3 n+13\right)$;

$(1,1,1,1)$

(.) $=\frac{1}{12} m^{4}+\frac{2}{3} m^{3} n+m^{2} n^{2}+\frac{1}{3} m n^{3}+\frac{1}{24} m^{4}$

$$
\begin{aligned}
& -\frac{1}{2} m^{3}-3 m^{2} n-2 m n^{2}-\frac{1}{4} n^{3}-\frac{181}{12} m^{2}-21 m n-\frac{229}{24} n^{2}+\frac{191}{2} m+\frac{403}{4} n \\
& +\alpha\left(-\frac{3}{2} m^{2}-3 m n-\frac{3}{4} n^{2}+\frac{43}{2} m+\frac{55}{4} n-\frac{357}{4}\right)+\alpha \cdot \frac{9}{8}
\end{aligned}
$$

$(/)=\frac{1}{24} m^{4}+\frac{1}{3} m^{3} n+m^{2} n^{2}+\frac{2}{3} m n^{3}+\frac{1}{12} n^{4}$

$$
\begin{aligned}
& -\frac{1}{4} m^{3}-2 m^{2} n-3 m n^{2}-\frac{1}{2} n^{3}-\frac{229}{24} m^{2}-21 m n-\frac{181}{2} n^{2}+\frac{403}{4} m+\frac{191}{2} n \\
& +\alpha\left(-\frac{3}{4} m^{2}-3 m n-\frac{3}{2} n^{2}+\frac{55}{4} m+\frac{43}{2} n-\frac{357}{4}\right)+\alpha^{2} \cdot \frac{9}{8}
\end{aligned}
$$

(2)

$$
\begin{aligned}
& (\therefore)=\alpha, \\
& (: /)=2 \alpha, \\
& (\cdot / /)=2 \alpha, \\
& (/ / /)=\alpha ;
\end{aligned}
$$

$(2,1)$

$$
\begin{aligned}
& (:)=12 m+12 n+(2 m+n-14) \alpha, \\
& (\cdot /)=24 m+24 n+(2 m+2 n-24) \alpha, \\
& (/ /)=12 m+12 n+(m+2 n-14) \alpha ;
\end{aligned}
$$

$(2,1,1)$

$$
\begin{aligned}
& (\cdot)=24 m^{2}+36 m n+12 n^{2}-168 m-168 n+\alpha\left(m^{2}+2 m n+\frac{1}{2} n^{2}-25 m-\frac{29}{2} n+138\right)-\frac{3}{2} \alpha^{2}, \\
& (/)=12 m^{2}+36 m n+24 n^{2}-168 m-168 n+\alpha\left(\frac{1}{2} m^{2}+2 m n+n^{2}-\frac{29}{2} m-25 n+138\right)-\frac{3}{2} \alpha^{2} ;
\end{aligned}
$$

$(2,2)$

$$
\begin{aligned}
& (\cdot)=27 m+24 n-20 \alpha+\frac{1}{2} \alpha^{2}, \\
& (/)=24 m+27 n-20 \alpha+\frac{1}{2} \alpha^{2} ;
\end{aligned}
$$

(3)

$$
\begin{aligned}
& (:)=-4 m-3 n+3 \alpha \\
& (\cdot /)=-8 m-8 n+6 \alpha \\
& (/ /)=-3 m-4 n+3 \alpha
\end{aligned}
$$


$(3,1)$

$$
\begin{aligned}
& (\cdot)=-8 m^{2}-12 m n-3 n^{2}+56 m+53 n+\alpha(6 m+3 n-39), \\
& (/)=-3 m^{2}-12 m n-8 n^{2}+53 m+56 n+\alpha(3 m+6 n-39) ;
\end{aligned}
$$

(4)

$$
\begin{aligned}
& (\cdot)=-10 m-8 n+6 \alpha \\
& (/)=-8 m-10 n+6 \alpha .
\end{aligned}
$$

50. By means of the foregoing formulæ I obtain, as will presently be shown, the following formulæ for the number of the conics which satisfy five conditions, viz. :

$$
(5)=-15 m-15 n+9 \alpha \text {; }
$$

$(4,1)=-8 m^{2}-20 m n-8 n^{2}+104 m+104 n+\alpha(6 m+6 n-66) ;$

$(3,2)=120 m+120 n+\alpha(-4 m-4 n-78)+3 \alpha^{2} ;$

$(3,1,1)=-\frac{3}{2} m^{3}-10 m^{2} n-10 m n^{2}-\frac{3}{2} n^{3}+\frac{109}{2} m^{2}+116 m n+\frac{109}{2} n^{2}-434 m-434 n$

$$
+\alpha\left(\frac{3}{2} m^{2}+6 m n+\frac{3}{2} n^{2}-\frac{69}{2} m-\frac{69}{2} n+291\right)-\frac{9}{2} \alpha^{2} ;
$$

$(2,2,1)=24 m^{2}+54 m n+24 n^{2}-468 m-468 n$

$$
+\alpha(-8 m-8 n+327)+\alpha^{2}\left(\frac{1}{2} m+\frac{1}{2} n-12\right) ;
$$

$(2,1,1,1)=6 m^{3}+30 m^{2} n+30 m n^{2}+6 n^{3}-174 m^{2}-348 m n-174 n^{2}+1320 m+1320 n$

$$
\begin{aligned}
& +\alpha\left(\frac{1}{6} m^{3}+m^{2} n+m n^{2}+\frac{1}{6} n^{3}-\frac{15}{2} m^{2}-26 m n-\frac{15}{2} n^{2}+\frac{358}{3} m+\frac{358}{3} n-960\right) \\
& +\alpha^{2}\left(-\frac{3}{2} m-\frac{3}{2} n+28\right)
\end{aligned}
$$

$(1,1,1,1,1)\left({ }^{1}\right)=\frac{1}{120} m^{5}+\frac{1}{12} m^{4} n+\frac{1}{3} m^{3} n^{2}+\frac{1}{3} m^{2} n^{3}+\frac{1}{12} m n^{4}+\frac{1}{120} n^{5}$

$$
\begin{aligned}
& -\frac{1}{12} m^{4}-\frac{5}{6} m^{3} n-2 m^{2} n^{2}-\frac{5}{6} m n^{3}-\frac{1}{12} n^{4} \\
& -\frac{113}{12} m^{3}-\frac{209}{12} m^{2} n-\frac{209}{12} m n^{2}-\frac{113}{25} n^{3} \\
& +\frac{1267}{12} m^{2}+\frac{593}{3} m n+\frac{1267}{12} n^{2}-\frac{3159}{5} m-\frac{3159}{5} n \\
& +\alpha\left(-\frac{1}{4} m^{3}-\frac{3}{2} m^{2} n-\frac{3}{2} m n^{2}-\frac{1}{4} n^{3}+\frac{29}{4} m^{2}+23 m n+\frac{29}{4} n^{2}-\frac{337}{4} m-\frac{337}{4} n+486\right) \\
& +\alpha^{2}\left(\frac{9}{8} m+\frac{9}{8} n-15\right) .
\end{aligned}
$$

51. I observe that by means of the above-mentioned expressions of $(X, 4 Z)$ and $(2 X, 3 Z)$, the foregoing results, other than those for $(5),(4,1)$, \&c., may be presented in a somewhat different form, viz. we have

$$
\text { (4Z) }(1)=n(\cdot)+m(/)
$$

where $(\cdot)$ denotes $(4 Z \cdot),(/)$ denotes $(4 Z /)$, and so in other cases, the understood term being $3 Z$ or $2 Z$, as the case may be.

\footnotetext{
1 In my paper in the Comptes Rendus, I gave erroneously the coefficients $-\frac{3259}{5} m-\frac{3259}{5} n \ldots+a\left(\ldots+\frac{195}{2}\right)$.
} 


$$
\begin{aligned}
(3 Z)(2)= & (\cdot /) \frac{1}{2} \alpha ; \\
(3 Z)(1,1)= & (:)\left(\frac{1}{2} n^{2}-\frac{1}{2} n\right) \\
& +(\cdot /)\left(m n-\frac{3}{4} \alpha\right) \\
& +(/ /)\left(\frac{1}{2} m^{2}-\frac{1}{2} m\right) \\
(2 Z)(3)= & (\therefore)\left(\frac{3}{2} m+n-\frac{3}{4} \alpha\right) \\
& +(: /)\left(-\frac{7}{4} m-\frac{3}{2} n+\frac{9}{8} \alpha\right) \\
& +(\cdot / /)\left(-\frac{3}{2} m-\frac{7}{4} n+\frac{9}{8} \alpha\right) \\
& +(/ / /)\left(m+\frac{3}{2} n-\frac{3}{4} \alpha\right) \\
& (\therefore)\left\{-3 m-3 n+\alpha\left(-\frac{1}{4} m+\frac{1}{4} n+2\right)\right\} \\
& +(: /)\left\{\frac{9}{2} m+\frac{9}{2} n+\alpha\left(\frac{3}{8} m+\frac{1}{8} n-4\right)\right\} \\
& +(\cdot / /)\left\{\frac{9}{2} m+\frac{9}{2} n+\alpha\left(\frac{1}{8} m+\frac{3}{8} n-4\right)\right\} \\
& +(/ / /)\left\{-3 m-3 n+\alpha\left(\frac{1}{4} m-\frac{1}{4} n+2\right)\right\}
\end{aligned}
$$

$(2 Z)(1,1,1)=$

$(\therefore)\left\{-\frac{1}{24} m^{3}-\frac{1}{4} m^{2} n+\frac{1}{4} m n^{2}+\frac{5}{24} n^{3}+\frac{1}{8} m^{2}+0 m n-\frac{5}{8} n^{2}+\frac{11}{12} m+\frac{29}{12} n+\alpha\left(\frac{3}{8} m-\frac{3}{8} n-1\right)\right\}$ $+(: /)\left\{\frac{1}{16} m^{3}+\frac{3}{8} m^{2} n+\frac{1}{8} m n^{2}-\frac{1}{16} n^{3}-\frac{3}{16} m^{2}-\frac{1}{2} m n+\frac{3}{16} n^{2}-\frac{19}{8} m-\frac{25}{8} n+\alpha\left(-\frac{9}{16} m-\frac{3}{16} n+3\right)\right\}$ $+(\cdot / /)\left\{\frac{1}{16} m^{3}+\frac{1}{8} m^{2} n+\frac{3}{8} m n^{2}+\frac{1}{16} n^{3}+\frac{3}{16} m^{2}-\frac{1}{2} m n-\frac{3}{16} n^{2}-\frac{25}{8} m-\frac{19}{8} n+\alpha\left(-\frac{3}{16} m-\frac{9}{16} n+3\right)\right\}$ $+(/ / /)\left\{\frac{5}{24} m^{3}+\frac{1}{4} m^{2} n-\frac{1}{4} m n^{2}-\frac{1}{24} n^{3}-\frac{5}{8} m^{2}+0 m n+\frac{1}{8} n^{2}+\frac{29}{12} m+\frac{11}{12} n+\alpha\left(-\frac{3}{8} m+\frac{3}{8} n-1\right)\right\}$; in all which formulæ it is to be recollected that we have

to which may be joined

$$
(\therefore)-\frac{3}{2}(: /)+\frac{3}{2}(\cdot / /)-(/ / /)=0 \text {, }
$$

$$
(Z)(4 X)=a(4 X \cdot)+b(4 X /)
$$

where $a, b$ are the representatives of the condition. $(Z)$, and where $(4 X)$ is to be considered as standing successively for $(4),(3,1),(2,2),(2,1,1)$, and $(1,1,1,1)$, the values of $(4 X \cdot)$ and $(4 X /)$ being in each case given by the foregoing Table.

52. The formulæ are very convenient for the calculation of the numbers of the conics which satisfy five conditions of contact with two given curves; thus if, for example, $(3 Z),=(3)_{m_{1}}$, denotes the condition of a contact of the third order with a given curve $\left(m_{1}\right)$, then writing for symmetry $(2)_{m}$ in place of $(2)$, we have

$$
\begin{aligned}
(3)_{m_{1}}(2)_{m} & =\frac{1}{2} \alpha(3 \cdot /)_{m_{1}} \\
& =\alpha\left(-4 m_{1}-4 n_{1}+3 \alpha_{1}\right) .
\end{aligned}
$$

53. To obtain the foregoing expressions of $(5),(4,1),(3,2),(3,1,1),(2,2,1)$, $(2,1,1,1)$, and $(1,1,1,1,1)$, I assume that the given curve breaks up into two curves $(m, n, \alpha)$ and $\left(m^{\prime}, n^{\prime}, \alpha^{\prime}\right)$, or, as we may for shortness express it, into two curves $m$ and $m^{\prime}$. 
We have then

$$
(5)_{m+m^{\prime}}=(5)_{m}+(5)_{m^{\prime}},
$$

viz. the conics which have contact of the 5th order with the aggregate curve $m+m^{\prime}$ are made up of the conics which have this contact with the curve $m$ and the conics which have this contact with the curve $m^{\prime}$. Writing this under the form

$$
(5)_{m+m^{\prime}}-(5)_{m}-(5)_{m^{\prime}}=0,
$$

and observing that $(5)_{m}$ is a function $\phi(m, n, \alpha)$, and that consequently this is a functional equation $\phi\left(m+m^{\prime}, n+n^{\prime}, \alpha+\alpha^{\prime}\right)-\phi(m, n, \alpha)-\phi\left(m^{\prime}, n^{\prime}, \alpha^{\prime}\right)=0$, the solution is

$$
\phi(m, n, \alpha)=a m+b n+c \alpha,
$$

where $a, b, c$ are arbitrary constants; but as the solution should be symmetrical in regard to $m$, n, we have $a=b$, or the solution is $\phi(m, n, \alpha)=a(m+n)+c \alpha$.

54. Similarly we have

$$
(4,1)_{m+m^{\prime}}-(4,1)_{m}-(4,1)_{m^{\prime}}=(4)_{m}(1)_{m^{\prime}}+(4)_{m^{\prime}}(1)_{m},
$$

viz. the conics which have with the aggregate curve $m+m^{\prime}$ the contacts $(4,1)$ are made up of the conics which have the two contacts 4 and 1 with the one curve or with the other curve, or the contact 4 with the one curve and the contact 1 with the other curve. The expression on the right-hand side is a known function of $(m, n, \alpha)$, $\left(m^{\prime}, n^{\prime}, \alpha^{\prime}\right)$; hence the form of the functional equation is

$$
\phi\left(m+m^{\prime}, n+n^{\prime}, \alpha+\alpha^{\prime}\right)-\phi(m, n, \alpha)-\phi\left(m^{\prime}, n^{\prime}, \alpha^{\prime}\right)=F\left(m, n, \alpha, m^{\prime}, n^{\prime}, \alpha^{\prime}\right) ;
$$

and any particular solution of this equation being obtained, the general solution is found by adding to it the term $a m+b n+c \alpha$. Assuming that the particular solution is symmetrical in regard to $(m, n)$, then the term to be added is as before $=a(m+n)+c \alpha$. And similarly for $(3,2),(3,1,1)$, \&c.; that is, in every case we have a solution containing two arbitrary constants $a$, $c$, which remain to be determined.

55. Now in every case except $(5)_{m}$ the number of intersections of the conic with the curve is $>6$ (viz. for $(4,1)_{m}$ and $(3,2)_{m}$ the number is 7 , for $(3,1,1)$ and $(2,2,1)$, it is 8 , and for the remaining two cases it is 9 and 10 respectively); hence if the given curve $m$ be a cubic, the number of conics satisfying the prescribed conditions is $=0$; and since a cubic may be the general cubic or a nodal or a cuspidal cubic, we have the three cases $(m, n, \alpha)=(3,6,18),(3,4,12)$, and $(3,3,10)$. We have thus in each case three conditions for the determination of the constants $a, c$; so that there is in each case a verification of the resulting formula.

56. In the omitted case $(5)_{m}$, when the curve $m$ is a cubic, the theory of the conics $(5)_{m}$ is a known one, viz. the points of contact of these conics, or the "sextactic" points of the cubic, are the points of contact of the tangents from the points of inflexion; the number of the conics $(5)_{m}$ is thus $=(n-3) \iota$, viz. in the three cases 
respectively it is $=27,3$, and 0 . Hence for determining the constants we have the three equations

$$
\begin{aligned}
& 9 a+18 c=27, \\
& 7 a+12 c=3, \\
& 6 a+10 c=0,
\end{aligned}
$$

which are satisfied by $a=-15, c=9$, and the resulting formula is

$$
(5)=-15 m-15 n+9 \alpha \text {. }
$$

In the particular case of a curve without nodes or cusps, this is $(5)=12 n-15 \mathrm{~m}$, $=m(12 m-27)$, which agrees with the result obtained in my memoir "On the Sextactic Points of a Plane Curve," Phil. Trans. vol. CLv. (1865), pp. 545-578, [341].

57. The subsidiary results required for the remaining cases $(4,1)$, \&c. are at once obtained from the foregoing formulæ for $(4 Z)(1),(3 Z)(2)$, \&c. ; for example, we have

$$
\begin{aligned}
(4)_{m}(1)_{m^{\prime}}= & n^{\prime}(-10 m-8 n+6 \alpha) \\
& +m^{\prime}(-8 m-10 n+6 \alpha),
\end{aligned}
$$

with like expressions for $(3,1)_{m}(1)_{m^{\prime}}$, \&c.,

$$
\begin{array}{rr}
(3)_{m}(2)_{m^{\prime}}= & \frac{1}{2} \alpha^{\prime}(-8 m-8 n+6 \alpha), \\
(3)_{m}(1,1)_{m^{\prime}}= & \left(\frac{1}{2} n^{\prime 2}-\frac{1}{2} n^{\prime}\right)(-4 m-3 n+3 \alpha) \\
& +\left(m^{\prime} n^{\prime}-\frac{3}{4} x^{\prime}\right)(-8 m-8 n+6 \alpha) \\
& +\left(\frac{1}{2} m^{\prime 2}-\frac{1}{2} m^{\prime}\right)(-3 m-4 n+3 \alpha) ;
\end{array}
$$

with like expressions for $(2,1)_{m}(2)_{m^{\prime}},(2,1)_{m}(1,1)_{m^{\prime}}$, \&c. \&c.

58. Calculation of $(4,1)$. We have

$$
\begin{aligned}
& (4,1)_{m+m^{\prime}}-(4,1)_{m}-(4,1)_{m^{\prime}}=(4)_{m}(1)_{m^{\prime}}+(4)_{m^{\prime}}(1)_{m}, \\
& \quad=-16 m m^{\prime}-20\left(m n^{\prime}+m^{\prime} n\right)-16 n n^{\prime}+6\left(\alpha n^{\prime}+\alpha^{\prime} n\right)+6\left(\alpha m^{\prime}+\alpha^{\prime} m\right),
\end{aligned}
$$

the integral of which is

$$
(4,1)_{m}=-8 m^{2}-20 m n-8 n^{2}+a(m+n)+\alpha(6 m+6 n+c) .
$$

The particular cases $(m, n, \alpha)=(3,6,18),(3,4,12),(3,3,10)$ give respectively

satisfied by $a=104, c=-66$.

$$
\begin{aligned}
& 0=252+9 a+18 c, \\
& 0=64+7 a+12 c, \\
& 0=36+6 a+10 c,
\end{aligned}
$$

c. VI. 
59. Calculation of $(3,2)$. We have

$$
\begin{aligned}
(3,2)_{m+m^{\prime}}-(3,2)_{m}-(3,2)_{m^{\prime}} & =(3)_{m}(2)_{m^{\prime}}+(3)_{m^{\prime}}(2)_{m}, \\
& =-4\left(m \alpha^{\prime}+m^{\prime} \alpha\right)-4\left(n \alpha^{\prime}+n^{\prime} \alpha\right)+6 \alpha \alpha^{\prime}:
\end{aligned}
$$

the integral is

and, as before,

$$
(3,2)_{m}=a(m+n)+\alpha(-4 m-4 n+c)+3 \alpha^{2},
$$

$$
\begin{gathered}
324+9 a+18 c=0 \\
96+7 a+12 c=0 \\
60+6 u+10 c=0
\end{gathered}
$$

satisfied by $a=120, c=-78$.

60 . For the calculation of $(3,1,1)$ we have similarly

$$
\begin{aligned}
(3,1,1)_{m+m^{\prime}}-(3,1,1)_{m}-(3,1,1)_{m^{\prime}}=(3)_{m}(1,1)_{m^{\prime}}+(3)_{m^{\prime}}(1,1)_{n} \\
+(3,1)_{m}(1)_{m^{\prime}}+(3,1)_{m^{\prime}}(1)_{m} .
\end{aligned}
$$

The function on the right-hand side was of course calculated from the values of $(3)_{m}(1,1)_{m}$, \&c.; but there is no use in this (and the more complicated cases which follow) in actually writing down the values of the function in question; it can in each case be calculated backwards from the foregoing expressions of $(3,1,1)$, \&c., and the values so obtained be verified by actual substitution. But assuming it to be known, the solution of the functional equation gives of course the foregoing expression for $(3,1,1)$, except that the terms in $m+n$ and $\alpha$ are therein $a(m+n)+c \alpha$; and I shall in this and the subsequent cases give only the three equations which determine the constants. In the present case these are

$$
\begin{aligned}
& -332+9 a+18 c=0 \\
& -454+7 a+12 c=0 \\
& -306+6 a+10 c=0,
\end{aligned}
$$

satisfied by $a=-434, c=291$.

61. The remaining cases are $(2,2,1),(2,1,1,1)$ and $(1,1,1,1,1)$. We have

$$
\begin{aligned}
(2,2,1)_{m+m^{\prime}}-(2,2,1)_{m^{\prime}}= & (2,2)_{m}(1)_{m^{\prime}}+(2,2)_{m^{\prime}}(1)_{m} \\
+ & (2,1)_{m}(2)_{m^{\prime}}+(2,1)_{m^{\prime}}(2)_{m},
\end{aligned}
$$

and

$$
\begin{aligned}
& -1674+9 a+18 c=0 \\
& -648+7 a+12 c=0 \\
& -462+6 a+10 c=0
\end{aligned}
$$

satisfied by $a=-468, c=327$.

Again,

$$
\begin{aligned}
(2,1,1,1)_{m+m^{\prime}}-(2,1,1,1)_{m}-(2,1,1,1)_{m^{\prime}}= & (2,1,1)_{m}(1)_{m^{\prime}}+(2,1,1)_{m^{\prime}}(1)_{m} \\
+ & (2,1)_{m}(1,1)_{m^{\prime}}+(2,1)_{m^{\prime}}(1)_{m} \\
& +(2)_{m}(1,1,1)_{m^{\prime}}+(2)_{m^{\prime}}(1,1,1)_{m}
\end{aligned}
$$


and

$$
\begin{aligned}
& 5400+9 a+18 c=0 \\
& 2280+7 a+12 c=0 \\
& 1680+6 a+10 c=0
\end{aligned}
$$

satisfied by $a=1320, c=-960$; and finally,

$$
\begin{aligned}
(1,1,1,1,1)_{m+m^{\prime}}-(1,1,1,1,1)_{m}-(1,1,1,1,1)_{m^{\prime}}= & (1,1,1,1)_{m}(1)_{m^{\prime}}+(1,1,1,1)_{m^{\prime}}(1)_{m} \\
+ & (1,1,1)_{m}(1,1)_{m^{\prime}}+(1,1,1)_{m^{\prime}}(1,1)_{m}
\end{aligned}
$$

and

$$
\begin{aligned}
& -30618+90 a+180 c=0 \\
& -14094+70 a+120 c=0 \\
& -10692+60 a+120 c=0
\end{aligned}
$$

satisfied by $10 a=6318,10 c=4860$, that is, $a=-\frac{3159}{5}, c=486$.

62. The contacts of a conic with a given curve which have been thus far considered are contacts at unascertained points of the curve; but a conic may have with the given curve at a given point thereof a contact of the first order, the condition will be denoted by $(\overline{2})$; or a contact of the second order, the condition will be denoted by $(\overline{3})$, and so on. It is to be observed that the conditions $(\overline{2}),(\overline{3})$, \&c. are sibireciprocal, the contact at a given point of the curve is the same thing as contact with a given tangent of the curve; but if we write $(\overline{1})$ to denote the condition of passing through a given point of the curve, this is not the same thing as the condition of tuuching a given tangent, of the curve; and this last condition, if it were necessary to deal with it, might be denoted by (1). But I attend only to the condition ( $\overline{1})$. The expressions for the number of conics which satisfy such conditions as $(\overline{\mathbf{1}}),(\overline{\mathbf{2}})$, \&c. are obtainable in several ways.

63. $\left(1^{\circ}\right)$ When the total number of conditions is 4 , the question may be solved by Zeuthen's method, viz. by determining the line-pairs and point-pairs of the system $4 Z$, with the proper numerical coefficients, and thence deducing the values of the characteristics $(4 Z \cdot)$ and $(4 Z /)$. A few cases are in fact thus solved in Zeuthen's work.

64. $\left(2^{\circ}\right)$ By the foregoing functional method. It is to be observed that there is a difference in the form of the functional equation, and that the general solution is always given in the form, Particular Solution + Constant, so that there is only a single constant to be determined by special considerations. To take the simplest example, let it be required to find the number of the conics $(3 Z)(\overline{1}, 1)$ : writing for shortness in place hereof $(\overline{1}, 1)$, or (in order to mark the curve $(m)$ to which the symbol has reference) $(\overline{1}, 1)_{m}$, let the curve $(m)$ be the aggregate of the curves $(m)$ and $\left(m^{\prime}\right)$. Regarding the point $\overline{1}$ as a given point on the curve $(m)$, that is, an arbitrary point in regard to the curve $\left(m^{\prime}\right)$, we have thus the equation

$$
(\overline{1}, 1)_{m+m^{\prime}}-(\overline{1}, 1)_{m}=(\cdot 1)_{m^{\prime}}
$$

where the right-hand side is known; and. so in general the form of the functional equation is always $\phi\left(m+m^{\prime}\right)-\phi(m)=$ given value, that is,

$$
\phi\left(m+m^{\prime}, n+n^{\prime}, \boldsymbol{\alpha}+\alpha^{\prime}\right)-\phi(m, n, \alpha)=\text { given function of }\left(m, n, \alpha, m^{\prime}, n^{\prime}, \alpha^{\prime}\right) ;
$$


whence, as stated, the general solution is Particular Solution + Constant. In the case in hand, taking successively $(3 Z)=(\therefore),(: /),(\cdot / /)$, and $(/ / /)$, we have in the first of these cases

$$
(\overline{1}, 1)_{m+m^{\prime}}-(\overline{1}, 1)_{m}=n^{\prime}+2 m^{\prime},
$$

whence $(\overline{1}, 1)_{m}=n+2 m+$ const. $=(\overline{1}, 1)(\therefore)$; and the value of the constant being in any way ascertained to be $=-2$, we have $(\overline{1}, 1)(\therefore)=n+2 m-2$; and the like for the other three cases.

65. ( $\left.3^{\circ}\right)$ The expressions for the number of conics which satisfy such conditions as $(\overline{\mathbf{1}}),(\overline{\mathbf{2}})$, \&c. are deducible with more or less facility from the corresponding expressions wherein $(\overline{\mathbf{1}}),(\overline{2})$, \&c. are replaced by $(\cdot),(:)$, \&c.; thus from $\left({ }^{1}\right)(:: 1)=n+2 m$ we deduce

$$
(\therefore \overline{1}, 1)=(:: /)-2(\therefore \overline{2})=n+2 m-2,
$$

viz. if one of the four arbitrary points of $(:: /)$ becomes a point on the curve, then the condition $(:: /)$ is satisfied specially by the conic $(\therefore \overline{2})$ which passes through the remaining three points and touches the curve at the point in question; 2 of the conics $(:: /)$ coincide with the conic in question. We have thus a reduction $2(\therefore \overline{2}),=2$, and the number of the conics $(\therefore \overline{1}, 1)$ is $=n+2 m-2$. Similarly, we have the system

$$
\begin{aligned}
& (\therefore \overline{1}, 1 \quad)=n+2 m-2, \\
& (: \overline{1}, \overline{1}, 1 \quad)=n+2 m-4, \\
& (\cdot \overline{1}, \overline{1}, \overline{1}, 1)=n+2 m-6, \\
& (\overline{1}, \overline{1}, \overline{1}, \overline{1}, 1)=n+2 m-8 .
\end{aligned}
$$

Again, two or even three of the given points on the curve may come together without any reduction being thereby caused, that is, we have

$$
\begin{aligned}
& (: \overline{2}, 1)=n+2 m-4 \text {, } \\
& (\cdot \overline{2}, \overline{1}, 1)=(\cdot \overline{3}, 1)=n+2 n-6 \text {, } \\
& (\overline{2}, \overline{1}, \overline{1}, 1)=(\overline{3}, \overline{1}, 1)=n+2 m-8 \text {; }
\end{aligned}
$$

but if the four points on the curve coincide in pairs, or, what is the same thing, if in $(\overline{2}, \overline{1}, \overline{1}, 1)$ the points $\overline{1}$ and $\overline{1}$ come to coincide, then there is a special reduction, and we have

$$
(\overline{2}, \overline{2}, 1)=n+2 m-8[-(m-2)]=m+n-6,
$$

viz. here $(m-2)$ of the conics come to coincide with the two points considered as a point-pair or infinitely thin conic. If the points $\overline{2}$ and $\overline{2}$ come to coincide, that is, if the four given points on the curve all coincide, there is no further reduction, but we have

$$
(\overline{4}, 1)=m+n-6 \text {. }
$$

1 I write indifferently (1) (::), $(1::)$ or $(:: 1)$; and so in other cases. 
66. The expressions involving a single ( $\overline{1})$ may in every case be reduced by the foregoing method to depend upon other expressions; thus we have

$$
\begin{aligned}
& (3 Z)(\overline{1}, 1) \quad=(\cdot 1) \quad-2(\overline{2}) \quad, \\
& (2 Z)(\overline{1}, 2) \quad=(\cdot 2) \quad-3(\overline{3}) \text {, } \\
& "(\overline{1}, 1,1)=(\cdot 1,1)-2(\overline{2}, 1), \\
& (Z)(\overline{1}, 1,2)=(\cdot 1,2)-2(\overline{2}, 2)-3(1, \overline{3}) \text {, } \\
& , \quad(\overline{1}, 1,1,1)=(\cdot 1,1,1)-2(\overline{2}, 1,1), \\
& "(\overline{1}, 3)=(\cdot 3) . \quad-4(\overline{4}) \text {, } \\
& (\overline{1}, 4) \quad=(\cdot 4) \quad-5(\overline{5}) \quad \text {, }
\end{aligned}
$$

where, comparing for example the equations for $(Z)(\overline{1}, 1,2)$ and $(2 Z)(\overline{1}, 1,1)$, it will be observed that in the first case the contacts 1,2 of the symbol $(\overline{1}, 1,2)$ successively coalesce with the point $\overline{1}$, giving respectively $2(\overline{2}, 2)$ and $3(1, \overline{3})$, the exterior factor being in each case the barred number, whereas the second case, where the contacts 1,1 of the symbol $(\overline{1}, 1,1)$ are of the same order, we do not consider each of these symbols separately (thus obtaining $2(\overline{2}, 1)+2(1, \overline{2}),=4(\overline{2}, 1)$ ), but the identical symbol is taken only once, giving $2(\overline{2}, 1)$. Thus we have also

$$
(\overline{1}, 1,1,1,1)=(\cdot 1,1,1,1)-2(\overline{2}, 1,1,1)
$$

67. The value of a symbol involving $(\overline{2})$, say the symbol $(3 Z)(\overline{2})$, is connected with that of $\frac{1}{2}(3 Z \cdot /)$; but as an instance of the correction which is sometimes required I notice the equation

$$
(\overline{2}, 1,1,1)=\frac{1}{2}(1,1,1 \cdot /)-\left\{\frac{1}{2}(m-2)(m-3)+\frac{1}{2}(n-2)(n-3)+3(\overline{3}, 1,1)+2(\overline{4}, 1)\right\},
$$

which I have verified by other considerations.

68. We obtain the series of results :

(1)

$$
\begin{aligned}
& (::)=1 \\
& (\therefore /)=2, \\
& (: / /)=4 \\
& (\cdot / / /)=4 \\
& (/ / /)=2 ;
\end{aligned}
$$

$(\overline{1}, 1)$

$$
\begin{aligned}
& (\therefore)=n+2 m-2, \\
& (: /)=2 n+4 m-4, \\
& (\cdot / /)=4 n+4 m-4, \\
& (/ / /)=4 n+2 m-2 ;
\end{aligned}
$$


$(\overline{1}, 2)$

$$
\begin{aligned}
& (:)=\alpha-3 \\
& (\cdot /)=2 \alpha-6 \\
& (/ /)=2 \alpha-3
\end{aligned}
$$

$(\overline{1}, 1,1)$

$$
\begin{aligned}
& (:)=2 m^{2}+2 m n+\frac{1}{2} n^{2}-6 m-\frac{5}{2} n+8-\frac{3}{2} \alpha \\
& (\cdot /)=2 m^{2}+4 m n+n^{2}-6 m-5 n+12-3 \alpha \\
& (/ /)=m^{2}+4 m n+2 n^{2}-3 m-5 n+8-3 \alpha
\end{aligned}
$$

$(\overline{1}, 3)$

$$
\begin{aligned}
& (\cdot)=-4 m-3 n-4+3 \alpha \\
& (/)=-8 m-8 n-4+6 \alpha ;
\end{aligned}
$$

$(\overline{1}, 1,2)$

$$
\begin{aligned}
& (\cdot)=6 m+9 n+30+\alpha(2 m+n-16) \\
& (/)=21 m+18 n+30+\alpha(2 m+2 n-26)
\end{aligned}
$$

$(\overline{1}, 1,1,1)$

( . $)=\frac{2}{3} m^{3}+2 m^{2} n+m n^{2}+\frac{1}{6} n^{3}-4 m^{2}-7 m n-\frac{3}{2} n^{2}+\frac{22}{3} m-\frac{2}{3} n-36+\alpha\left(-3 m-\frac{3}{2} n+16\right)$,

( / ) $=\frac{1}{3} m^{3}+2 m^{2} n+2 m n^{2}+\frac{1}{3} n^{3}-2 m^{2}-8 m n-3 n^{2}-\frac{19}{3} m-\frac{4}{3} n-36+\alpha(-3 m-3 n+23)$;

$(\overline{2})$

$$
\begin{aligned}
& (\therefore)=1, \\
& (: /)=2, \\
& (\cdot / /)=2, \\
& (/ / /)=1 ;
\end{aligned}
$$

$(\overline{2}, 1)$

$$
\begin{aligned}
& (:)=2 m+n-4, \\
& (\cdot /)=2 m+2 n-6, \\
& (/ /)=m+2 n-4 ;
\end{aligned}
$$

$(\overline{2}, 2)$

$$
\begin{aligned}
& (\cdot)=a-6, \\
& (/)=a-6 ;
\end{aligned}
$$

$(\overline{2}, 1,1)$

$$
\begin{aligned}
& (\cdot)=m^{2}+2 m n+\frac{1}{2} n^{2}-7 m-\frac{9}{2} n+18-\frac{3}{2} \alpha, \\
& (/)=\frac{1}{2} m^{2}+2 m n+n^{2}-\frac{9}{2} m-7 n+18-\frac{3}{2} \alpha ;
\end{aligned}
$$

$(\overline{3})$

$$
\begin{aligned}
& (:)=1, \\
& (\cdot /)=2, \\
& (/ /)=1 ;
\end{aligned}
$$


$(\overline{3}, 1)$

$$
\begin{array}{ll}
(\cdot) & =n+2 m-6 \\
(/) & =2 n+m-6 ;
\end{array}
$$

$(\overline{4})$

$$
\begin{array}{ll}
(\cdot) & =1 \\
(/) & =1 ;
\end{array}
$$

which are the several cases for the conics which satisfy not more than four conditions, and

69. For the conics satisfying 5 conditions, we have

$$
(\overline{4}, 1)=m+n-6 \text {, }
$$$$
(\overline{3}, 2) \quad=-9+\alpha \text {, }
$$$$
(\overline{3}, 1,1) \quad=\frac{1}{2} m^{2}+2 m n+\frac{1}{2} n^{2}-\frac{13}{2} m-\frac{13}{2} n+27-\frac{3}{2} \alpha,
$$$$
(\overline{2}, 3) \quad=-4 m-4 n-6+3 \alpha \text {, }
$$$$
(\overline{2}, 2,1)=6 m+6 n+54+\alpha(m+n-15) \text {, }
$$$$
(\overline{2}, 1,1,1)=\frac{1}{6} m^{3}+m^{2} n+m n^{2}+\frac{1}{6} n^{3}-\frac{5}{2} m^{2}-8 m n-\frac{5}{2} n^{2}+\frac{37}{3} m+\frac{37}{3} n-75
$$

$$
+\alpha\left(-\frac{3}{2} m-\frac{3}{2} n+\frac{29}{2}\right) \text {, }
$$

$(\overline{1}, 4) \quad=-10 m-8 n-5+6 \alpha$,

$(\overline{1}, 1,3)=-8 m^{2}-12 m n-3 n^{2}+60 m+57 n+36+\alpha(6 m+3 n-45)$,

$(\overline{1}, 2,2) \quad=27 m+24 n+27-23 \alpha+\frac{1}{2} \alpha^{2}$,

$(\overline{1}, 1,1,2)=\frac{45}{2} m^{2}+30 m n+\frac{21}{2} n^{2}-\frac{321}{2} m-\frac{321}{2} n-189$

$$
+\alpha\left(m^{2}+2 m n+\frac{1}{2} n^{2}-27 m-\frac{33}{2} n+\frac{345}{2}\right)-\frac{3}{2} \alpha^{2},
$$

$(\overline{1}, 1,1,1,1)=\frac{1}{12} m^{4}+\frac{2}{3} m^{3} n+m^{2} n^{2}+\frac{1}{3} m n^{3}+\frac{1}{24} n^{4}-\frac{5}{6} m^{3}-5 m^{2} n-4 m n^{2}-\frac{7}{2} n^{3}$

$$
\begin{aligned}
& -\frac{121}{12} m^{2}-5 m n-\frac{209}{4} n^{2}+\frac{425}{6} m+\frac{913}{4} n+150 \\
& +\alpha\left(-\frac{3}{2} m^{2}-3 m n-\frac{3}{4} n^{2}+\frac{49}{2} m+\frac{67}{4} n-\frac{473}{4}\right)+\frac{9}{8} \alpha^{2} .
\end{aligned}
$$

70. The given point on the curve to which the symbols $\overline{1}, \overline{2}$, \&c. refer may be a singular point, and in particular it is proper to consider the case where the point is a cusp. I use in this case an appropriate notation; a conic which simply passes through a cusp, in fact meets the curve at the cusp in two points; and I denote the condition of passing through the cusp by $1 \kappa 1$; similarly, a conic which touches the curve at the cusp, in fact there meets it in three points, and I denote the condition by $\overline{2 \kappa 1} ; \overline{1 \kappa 1}, \overline{2 \kappa 1}$ are thus special forms of $\overline{1}, \overline{2}$, and the annexed $\overline{1}$ indicates the additional point of intersection arising ipso facto from the point $\overline{1}$ or $\overline{2}$ being a cusp. Similarly, we should have the symbols $3 \kappa 1, \overline{4 \kappa 1}, 5 \kappa 1$; but it is to be observed that at a cusp of the curve there is no proper conic having a higher contact than 
$\overline{2 \kappa 1}$; thus if the symbol contains $\overline{3 \kappa 1}$, or $\grave{a}$ fortiori, if it contain $\overline{4 \kappa 1}$ or $\overline{5 \kappa 1}$, the number of the conics is in every case $=0$; it is thus only the cases $1 \kappa 1$ and $2 \kappa 1$ which need to be considered.

71. The several modes of investigation which apply to the case of contact at a given ordinary point of the curve are applicable to the case of contact at a cusp: we may if we please employ the functional method; we have here a functional equation of the foregoing form, $\phi\left(m+m^{\prime}\right)-\phi m=$ given value (that is, $\phi\left(m+m^{\prime}, n+n^{\prime}, \alpha+\alpha^{\prime}\right)$ $-\phi(m, n, \alpha)=$ given function of $\left.\left(m, n, \alpha, m^{\prime}, n^{\prime}, \alpha^{\prime}\right)\right)$, and the general solution is as before = Particular Solution + Constant; so that there is in each case a single arbitrary constant to be determined by special considerations. The determination of the constant is in some instances conveniently effected by means of the case of the cuspidal cubic: see Annexes Nos. 4 and 5 .

The formation of the functional equation itself is similar to that in the corresponding case where the given point on the curve is an ordinary point. For example, we have

$$
\begin{aligned}
&(2 Z)(1, \overline{1}, 1)_{m+m^{\prime}}-(1, \overline{1}, 1)_{m}=(1, \overline{1})_{m}(1)_{m^{\prime}}= \\
&+(\overline{1})_{m}(1,1)_{m^{\prime}}(\overline{1}, 1 \cdot)_{m}+m^{\prime}(\overline{1}, 1 /)_{m} \\
&+\frac{1}{2}\left(n^{\prime 2}-n^{\prime}\right)(\overline{1}:)_{m} \\
&+\left(m^{\prime} n^{\prime}-\frac{3}{4} \alpha^{\prime}\right)(\overline{1} \cdot /)_{m} \\
&+\frac{1}{2}\left(m^{\prime 2}-m^{\prime}\right)(\overline{1} / /)_{m},
\end{aligned}
$$

and we may herein simply change $\overline{1}$ intc $1 \kappa 1$. Writing successively $2 Z=(:),(\cdot /)$ and $(/ /)$, we find

$(1 \kappa 1,1,1:)_{m+m^{\prime}}-(:)_{m}=n^{\prime}(n+2 m-3)+m^{\prime}(2 n+2 m-6)+\left(\frac{1}{2} n^{\prime 2}-\frac{1}{2} n^{\prime}\right) 1+\left(m^{\prime} n^{\prime}-\frac{3}{4} \alpha^{\prime}\right) 2+\left(\frac{1}{2} m^{\prime 2}-\frac{1}{2} m^{\prime}\right) 4$,

$\cdot /)_{m+m^{\prime}}-(\cdot /)_{m}=n^{\prime}(2 n+4 m-6)+m^{\prime}(4 n+4 m-6)+\left(\frac{1}{2} n^{\prime 2}-\frac{1}{2} n^{\prime}\right) 2+\left(m^{\prime} n^{\prime}-\frac{3}{4} \alpha^{\prime}\right) 4+\left(\frac{1}{2} m^{\prime 2}-\frac{1}{2} m^{\prime}\right) 4$

$/ /)_{m+m^{\prime}}-(/ /)_{m}=n^{\prime}(4 n+4 m-6)+m^{\prime}(4 n+2 m-3)+\left(\frac{1}{2} n^{\prime 2}-\frac{1}{2} n^{\prime}\right) 4+\left(m^{\prime} n^{\prime}-\frac{3}{4} \alpha^{\prime}\right) 4+\left(\frac{1}{2} m^{\prime 2}-\frac{1}{2} m^{\prime}\right) 4$,

which only differ from the corresponding expressions with $\overline{\mathbf{1}}$ in that they contain

in place of

$$
n+2 m-3,2 n+4 m-6,4 n+4 m-6,4 m+2 n-3
$$

$$
n+2 m-2,2 n+4 m-4,4 n+4 m-4,4 m+2 n-2
$$

respectively, and they lead to the expressions for $(1 \kappa 1,1,1:)$, \&c., the arbitrary constant being in each case properly determined.

72. We have

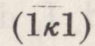

$$
\begin{aligned}
& (::)=1, \\
& (\therefore /)=2, \\
& (: / /)=4, \\
& (\cdot / / /)=4, \\
& (/ / / /)=2 ;
\end{aligned}
$$


$(1 \kappa 1,1)$

$$
\begin{aligned}
& (\therefore)=n+2 m-3, \\
& (: /)=2 n+4 m-6, \\
& (\cdot / /)=4 n+4 m-6, \\
& (/ / /)=4 n+2 m-3
\end{aligned}
$$

$(\overline{1} \kappa 1,2)$

$$
\begin{aligned}
& (:)=\alpha-4 \\
& (\cdot /)=2 \alpha-8 \\
& (/ /)=2 \alpha-4
\end{aligned}
$$

$(\overline{1 \kappa} 1,1,1)$

$$
\begin{aligned}
& (:)=2 m^{2}+2 m n+\frac{1}{2} n^{2}-8 m-\frac{7}{2} n+13-\frac{3}{2} \alpha \\
& (\cdot /)=2 m^{2}+4 m n+n^{2}-8 m-7 n+18-3 \alpha \\
& (/ /)=m^{2}+4 m n+2 n^{2}-4 m-8 n+12-3 \alpha
\end{aligned}
$$

$(\overline{1 \kappa 1}, 3)$

$$
\begin{aligned}
& (\cdot)=-4 m-3 n-5+3 \alpha \\
& (/)=-8 m-8 n-6+6 \alpha ;
\end{aligned}
$$

$(\overline{1 \kappa} 1,1 ; 2)$

$$
\begin{aligned}
& (\cdot)=4 m+8 n+44+\alpha(2 m+n-17) \\
& (/)=20 m+16 n+42+\alpha(2 m+2 n-27)
\end{aligned}
$$

$(\overline{1} \kappa 1,1,1,1)$

$$
\begin{aligned}
& \text { ( . })=\frac{2}{3} m^{3}+2 m^{2} n+m n^{2}+\frac{1}{6} n^{3}-5 m^{2}-9 m n-2 n^{2}+\frac{43}{3} m+\frac{29}{6} n-57+\alpha\left(-3 m-\frac{3}{2} n+\frac{35}{2}\right), \\
& (\quad)=\frac{1}{3} m^{3}+2 m^{2} n+2 m n^{2}+\frac{1}{3} n^{3}-\frac{5}{2} m^{2}-10 m n-4 n^{2}-\frac{11}{6} m+\frac{17}{3} n-54+\alpha\left(-3 m-3 n+\frac{49}{2}\right) ;
\end{aligned}
$$

$(2 \kappa 1)$

$$
\begin{aligned}
& (\therefore)=1, \\
& (: /)=2, \\
& (\cdot / /)=2, \\
& (/ / /)=1,
\end{aligned}
$$

$(2 \kappa 1,1)$

$$
\begin{aligned}
& (:)=2 m+n-5 \\
& (\cdot /)=2 m+2 n-6 \\
& (/ /)=m+2 n-4
\end{aligned}
$$

$(\overline{2 \kappa 1}, 2)$

$$
\begin{aligned}
& (\cdot)=\alpha-7, \\
& (/)=\alpha-6 ;
\end{aligned}
$$

c. VI. 
$(\overline{2 \kappa 1}, 1,1)$

$$
\begin{aligned}
& \text { ( ) }=m^{2}+2 m n+\frac{1}{2} n^{2}-7 m-\frac{11}{2} n+21-\frac{3}{2} \alpha, \\
& (/)=\frac{1}{2} m^{2}+2 m n+n^{2}-\frac{9}{2} m-7 n+18-\frac{3}{2} \alpha .
\end{aligned}
$$

73. The remainder of this table, being the part where the symbols $(\cdot)$ and $(/)$ do not occur, I present under a somewhat different form as follows:

$$
\begin{aligned}
& (5 \kappa 1)=0 \text {, } \\
& (\overline{4 \kappa 1}, 1) \quad=0 \text {, } \\
& (3 \kappa 1,2)=0 \text {, } \\
& (3 \kappa 1,1,1)=0 \text {, } \\
& (\overline{2}, 3) \quad-(\overline{2 \kappa 1}, 3) \quad=0 \text {, } \\
& (\overline{2}, 2,1) \quad-(\overline{2 \kappa 1}, 2,1) \quad=n-3 \text {, } \\
& (\overline{2}, 1,1,1) \quad-(2 \kappa 1,1,1,1)=\frac{1}{2}(n-3)(n-4), \\
& (\overline{1}, 4) \quad-(\overline{1 \kappa 1}, 4)=1 \text {, } \\
& (\overline{1}, 1,3) \quad-(\overline{1 \kappa 1}, 1,3) \quad=(2 \kappa 1,3)+(n-3) \text {, } \\
& (\overline{1}, 2,2) \quad-(\overline{1 \kappa 1}, 2,2) \quad=3(n-3)+\kappa-1 \text {, } \\
& (\overline{1}, 1,1,2)-(1 \kappa 1,1,1,2)=(2 \kappa 1,1,2)+\frac{1}{2}(n-3)(n-4)+\delta+2 n-3 m-4 \text {, } \\
& (\overline{1}, 1,1,1,1)-(\overline{1 \kappa 1}, 1,1,1,1)=(\overline{2 \kappa 1}, 1,1,1) \text {. }
\end{aligned}
$$

These results relating to a cusp, are useful for the investigations contained in the Second Memoir.

It will be noticed that the symbols which contain $2 \kappa 1$ are not, like those which contain $\overline{2}$, symmetrical in regard to $(m, n)$ : the interchange of $(m, n)$ would of course imply the change of a cusp into an inflexion, and would therefore give rise to a new symbol such as $2 \imath 1$; but I have not thought it necessary to consider the formulæ which contain this new symbol.

Investigations in extension of those of $\mathrm{DE}$ JoNQUIÈRES in relation to the contacts of a Curve of the order $\mathrm{r}$ with a given curve. Article Nos. 74 to 93.

74. De Jonquières has given a formula for the number of curves $C^{r}$ of the order $r$ which have with a given curve $U^{m}$ of the $m$ th order $t$ contacts of the orders $a, b, c$, \&c. respectively, which besides pass through $p$ points distributed at pleasure on the curve $U^{m}$ (this includes the case of contacts of any orders at given points of the curve $\left.U^{m}\right)$, and which moreover satisfy any other $\frac{1}{2} r(r+3)-(a+b+c+\& c)-$. 
conditions; viz. the number of the curves $C^{r}$ is $=\mu(a+1)(b+1)(c+1) \ldots$ into

$$
\left\{\begin{array}{l}
{[r m-(a+b+c \ldots)-p]^{t}} \\
+[r m-(a+b+c \ldots)-p-1]^{t-1}(a+b+c \ldots)[D]^{1} \\
+[r m-(a+b+c \ldots)-p-2]^{t-2}(a b+a c+b c \ldots)[D]^{2} \\
\quad: \quad(r m-(a+b+c \ldots)-p-t]^{\circ}(a b c \ldots) \quad[D]^{t},
\end{array}\right.
$$

where the curve $U^{m}$ is a curve without cusps, and having therefore a deficiency $D=\frac{1}{2}(m-1)(m-2)-\delta$; the numbers $a, b, c, \ldots$ are assumed to be all of them unequal, but if we have $\alpha$ of them each $=a, \beta$ of them each $=b$, \&c., then the foregoing expression is to be divided by $[\alpha]^{\alpha}[\beta]^{\beta} \ldots$; and $\mu$ denotes the number of the curves $C^{r}$ which satisfy the system of conditions obtained from the given system by replacing the conditions of the $t$ contacts of the orders $a, b, c$, \&c. respectively by the condition of passing throing $a+b+c \ldots$ arbitrary points. In order that the formula may give the number of the proper curves $C^{r}$ which satisfy the prescribed conditions, it is sufficient that the $\frac{1}{2} r(r+3)-(a+b+c \ldots)-p$ conditions shall include the conditions of passing through at least a certain number $T$ of arbitrary points: this restriction applies to all the formulæ of the present section.

75. I will for convenience consider this formula under a somewhat less general form, viz. I will put $p=0$, and moreover assume that the $\frac{1}{2} r(r+3)-(a+b+c \ldots)$ conditions are the conditions of passing through this number of arbitrary points; whence $\mu=1$.

We have thus a curve $C^{r}$ having with the given curve $U^{m} t$ contacts of the orders $a, b, c \ldots$ respectively, and besides passing through $\frac{1}{2} r(r+3)-(a+b+c \ldots)$ arbitrary points; and the number of such curves is by the formula $=(a+1)(b+1)(c+1), \ldots$ into

$$
\left\{\begin{array}{c}
{[r m-(a+b+c . .) \quad]^{t}} \\
+[r m-(a+b+c . .)-1]^{t-1}(a+b+c \ldots)[D]^{1} \\
+[r m-(a+b+c . .)-2]^{t-2}(a b+a c+b c . .)[D]^{2} \\
\vdots \\
+[r m-(a+b+c . .)-t]^{\circ}(a b c \ldots) \quad[D]^{t}
\end{array}\right.
$$

where, as before, in the case of any equalities between the numbers $a, b, c, \ldots$, the expression is to be divided by $[\alpha]^{\alpha}[\beta]^{\beta} \ldots$.

76. I have succeeded in extending the formula to the case of a curve with cusps: instead of writing down the general formula, I will take successively the cases of a single contact $a$, two contacts $a, b$, three contacts $a, b, c$, \&c.; and then denoting the numbers of the curves $C^{r}$ by $(a),(a, b),(a, b, c)$, \&c. in these cases respectively, I say that we have

$$
\begin{array}{r}
(a)=\quad(a+1)\left\{\begin{array}{c}
r m-a \\
+a D
\end{array}\right\} \\
-a . . \kappa
\end{array}
$$




$$
\begin{aligned}
& (a, b)=\quad(a+1)(b+1)\left\{\begin{array}{ll}
{[r m-a-b]^{2}} & \\
+[r m-a-b-1]^{1} & (a+b)[D]^{1} \\
+ & a b \quad[D]^{2}
\end{array}\right\} \\
& \left\{\begin{array}{c}
a(b+1)\left\{\begin{array}{l}
{[r m-a-b-1]^{1}} \\
+
\end{array}\right\} \\
+b(a+1)\left\{\begin{array}{l}
{[r m-a-b-1]^{1}} \\
+
\end{array}\right\}
\end{array}\right\}^{[\kappa]^{1}} \\
& +a b \\
& (a, b, c)=(a+1)(b+1)(c+1)\left\{\begin{array}{c}
{[r m-a-b-c]^{3}} \\
+[r m-a-b-c-1]^{2}(a+b+c)[D]^{1} \\
+[r m-a-b-c-2]^{1}(a b+a c+b c)[D]^{2} \\
+a b c
\end{array}\right\} \\
& -\left[\Sigma c ( a + 1 ) ( b + 1 ) \left\{\begin{array}{l}
{[r m-a-b-c-1]^{2}} \\
+[r m-a-b-c-2]^{1}(a+b)[D] \\
+\quad a b \quad[D]
\end{array}\right.\right. \\
& +\left[\Sigma b c ( a + 1 ) \quad \left\{\begin{array}{l}
{[r m-a-b-c-2]^{1}} \\
+\quad a D
\end{array}\right.\right. \\
& \}^{][\kappa]^{2}} \\
& -a b c
\end{aligned}
$$

$[\kappa]^{3}$.

77. The foregoing examples are sufficient to exhibit the law; but as I shall have to consiàer the cases of four and five contacts, I will also write down the formula for $(a, b, c, d)$, putting therein for shortness

$$
\begin{aligned}
& a+b+c+d=\alpha, a b+\ldots+c d=\beta, a b c .+b c d=\gamma, a b c d=\delta, \\
& a+b+c=\alpha^{\prime}, a b+a c+b c=\beta^{\prime}, a b c=\gamma^{\prime}, a+b=\alpha^{\prime \prime}, a b=\beta^{\prime \prime}, a=\alpha^{\prime \prime \prime}
\end{aligned}
$$

and also the formula for $(a, b, c, d, e)$, putting therein in like manner

$$
(\alpha, \beta, \gamma, \delta, \epsilon),\left(\alpha^{\prime}, \beta^{\prime}, \gamma^{\prime}, \delta^{\prime}\right),\left(\alpha^{\prime \prime}, \beta^{\prime \prime}, \gamma^{\prime \prime}\right),\left(\alpha^{\prime \prime \prime}, \beta^{\prime \prime \prime}\right),\left(\alpha^{\prime \prime \prime \prime}\right)
$$

for the combination, of $(a, b, c, d, e),(a, b, c, d),(a, b, c),(a, b)$ and $(a)$ respectively. We have

$$
(a, b, c, d)=(a+1)(b+1)(c+1)(d+1)\left\{\begin{array}{c}
{[r m-\alpha]^{4}} \\
+[r m-\alpha-1]^{3} \alpha[D]^{1} \\
+[r m-\alpha-2]^{2} \beta[D]^{2} \\
+[r m-\alpha-3]^{1} \gamma[D]^{3} \\
+\quad \delta[D]^{4}
\end{array}\right\}
$$




$$
\begin{aligned}
& {\left[\Sigma d(a+1)(b+1)(c+1)\left\{\begin{array}{c}
{[r m-\alpha-1]^{3}} \\
+[r m-\alpha-2]^{2} \alpha^{\prime}[D]^{1} \\
+[r m-\alpha-3]^{1} \beta^{\prime}[D]^{2} \\
+\quad \gamma^{\prime}[D]^{3}
\end{array}\right\}\right.} \\
& +\left[\Sigma c d \cdot(a+1)(b+1) \quad\left\{\begin{array}{c}
{[r m-\alpha-2]^{2}} \\
+[r m-\alpha-3]^{1} \alpha^{\prime \prime}[D]^{1} \\
+\quad \beta^{\prime \prime}[D]^{2}
\end{array}\right\}\right. \\
& -\left[\Sigma b c d(a+1) \quad\left\{\begin{array}{ll}
{[r m-\alpha-3]^{1}} \\
+ & \alpha^{\prime \prime \prime}[D]^{1}
\end{array}\right\}^{[\kappa]^{3}}\right. \\
& +a b c d \text {. . . . . . . }[\kappa]^{4} \text {, }
\end{aligned}
$$

$$
\begin{aligned}
& (a, b, c, d, e)=(a+1)(b+1)(c+1)(d+1)(e+1)\left\{\begin{array}{ccc}
{[r m-\alpha} & ]^{5} & \\
+[r m-\alpha-1]^{4} \alpha & {[D]^{1}} \\
+[r m-\alpha-2]^{3} \beta & {[D]^{2}} \\
+[r m-\alpha-3]^{2} \gamma & {[D]^{3}} \\
+[r m-\alpha-4]^{1} & \delta & {[D]^{4}} \\
+ & \epsilon & {[D]^{5}}
\end{array}\right\} \\
& -[\Sigma e(a+1)(b+1)(c+1)(d+1)
\end{aligned}
$$

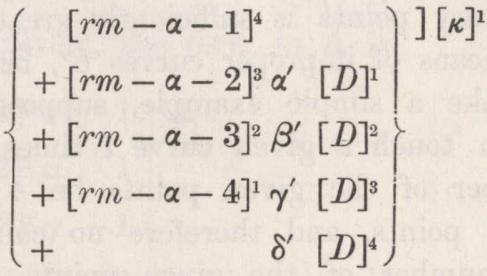

$$
\begin{aligned}
& +[\Sigma d e(a+1)(b+1)(c+1) \\
& \left\{\begin{array}{c}
{[r m-\alpha-2]^{3}} \\
+[r m-\alpha-3]^{2} \alpha^{\prime \prime}[D]^{1} \\
+[r m-\alpha-4]^{1} \beta^{\prime \prime}[D]^{2} \\
+\quad \gamma^{\prime \prime}[D]^{3}
\end{array}\right\} \\
& -[\Sigma c d e(a+1)(b+1) \\
& \left\{\begin{array}{c}
{[r m-\alpha-3]^{2}} \\
+[r m-\alpha-4]^{1} \alpha^{\prime \prime \prime}[D]^{1} \\
+\quad \beta^{\prime \prime \prime}[D]^{2}
\end{array}\right\} \\
& +[\Sigma b c d e(a+1) \\
& \left\{\begin{array}{cc}
{[r m-\alpha-4]^{1}} & \alpha^{\prime \prime \prime \prime}[D]^{1}
\end{array}\right\}^{][\kappa]^{4}}
\end{aligned}
$$

- abcde.

$[\kappa]^{5}$. 
78. In all these formulæ there is, as before, a numerical divisor in the case of any equalities among the numbers $a, b, c$, \&c. And $D$ denotes, as before, the deficiency, viz. its value now is $D=\frac{1}{2}(m-1)(m-2)-\delta-\kappa$; or observing that the class $n$ is $=m^{2}-m-2 \delta-3 \kappa$, we have $D=\frac{1}{2} n-m+1+\frac{1}{2} \kappa$, or say $D=1-m+\frac{1}{2} n+\frac{1}{2} \kappa,=1+\Delta$ if $\Delta=-m+\frac{1}{2} n+\frac{1}{2} \kappa$.

79. It is to be observed with reference to the applicability of these formulæ within certain limits only, that the formulæ are the only formulæ which are generally true; thus taking the simplest case, that of a single contact $a$, the only algebraical expression for the number of the curves $C^{r}$ which have with a given curve $U^{m}$ a contact of the order $a$, and besides pass through the requisite number $\frac{1}{2} r(r+3)-a$ of arbitrary points, is that given by the formula, viz.

$$
(a)=(a+1)(r m-a+a D)-a \kappa .
$$

Considering the curve $U^{m}$ and the order $r$ of the curve $C^{r}$ as given, if $a$ has successively the values $1,2, \ldots$ up to a limiting value of $a$, the formula gives the number of the proper curves $C^{r}$ which have with the given curve $U^{m}$ a contact of the required order $a$ : beyond this limiting value the formula no longer gives the number of the proper curves $C^{r}$ which satisfy the required condition, and it thus ceases to be applicable; but there is no algebraic function of $a$ which would give the number of the proper curves $C^{b r}$ as well beyond as up to the foregoing limiting value of $a$.

80. The formulæ are applicable pruvided only the conditions include the conditions of passing through a sufficient number of arbitrary points; viz. when the number of arbitrary points is sufficiently great, it is not possible to satisfy the conditions specially by means of improper curves $C^{r}$, being or comprising a pair of coincident curves. Thus to take a simple example, suppose it is required to find the number of the conics which touch a given curve $t$ times and besides pass through $5-t$ given points: if the number of the given points be 4 or 3 there is no coincident line-pair through the given points, and therefore no coincident line-pair satisfying the given conditions; if the number of the given points is $=2$, then the line joining these points gives a coincident line-pair having at each of its $m$ intersections with the given curve a special contact therewith, that is, having in $\frac{1}{6} m(m-1)(m-2)$ ways three special contacts with the given curve; if the number of the given points is 1 or 0 , then in the first case any line whatever through the given point, and in the second case any line whatever, regarded as a coincident line-pair, has $m$ special contacts with the given curve; and so in general there is a certain value for the number of given points, for which value the conditions of contact may be satisfied by a determinate number of improper curves $C^{r}$, and for values inferior to it the conditions may be satisfied by infinite series of improper curves $C^{r}$. It is by such considerations as these that De Jonquières has determined the minimum value $T$ of the number of arbitrary points to which the conditions should relate in order that the formulæ may be applicable: I refer for his investigation and results to paragraphs XVII and XVIII of his memoir. I remark that in the case where the number of improper solutions is finite, the formula can be corrected so as to give the number of proper solutions by simply 
subtracting the number of the improper solutions: but this is not so when the improper solutions are infinite in number; the mode of obtaining the approximate formula is here to be sought in the considerations contained in the first part of the present Memoir; see in particular ante, Nos. 8, 9 and 10.

81. The expressions for $(a),(a, b)$, \&c. may be considered as functions of $r m, 1+\Delta$, and $\kappa$, and they vanish upon writing therein $r m=0, \Delta=0, \kappa=0$; they are consequently of the form $(r m, \Delta, \kappa)^{1}+(r m, \Delta, \kappa)^{2}+\& c$., and I represent by $[a],[a, b]$, \&c. the several terms $(r m, \Delta, \kappa)^{1}$, which are the portions of $(a),(a, b)$, \&c. respectively, linear in $r m, \Delta$, and $\kappa$. The terms in question are obtained with great facility; thus, to fix the ideas, considering the expressions for $(a, b, c, d)$,

$1^{\circ}$. To obtain the term in $r m$, we may at once write $D=1, \kappa=0$, the expression is thus reduced to

$$
(a+1)(b+1)(c+1)(d+1)\left\{[r m-\alpha]^{4}+[r m-\alpha-1]^{3} \alpha\right\},
$$

and the factor in \{\} being $=r m[r m-\alpha-1]^{3}$, the coefficient of $r m$ is

which is

$$
(a+1)(b+1)(c+1)(d+1)[-\alpha-1]^{3},
$$

$$
=-(a+1)(b+1)(c+1)(d+1) \cdot(\alpha+1)(\alpha+2)(\alpha+3) .
$$

$2^{\circ}$. To obtain the term in $\Delta$, writing $r m=0, \kappa=0$, and observing that

$$
[D]^{1}=\Delta+1, \quad[D]^{2}=(\Delta+1) \Delta, \quad[D]^{3}=(\Delta+1) \Delta(\Delta-1), \quad[D]^{4}=(\Delta+1) \Delta(\Delta-1)(\Delta-2),
$$

\&c. give the terms $\Delta, \Delta,-\Delta,+2 \Delta,-6 \Delta$, \&c. respectively, the term in $\Delta$ is



$$
\begin{aligned}
& =(a+1)(b+1)(c+1)(d+1)\left\{\begin{array}{lr}
-\alpha(\alpha+1)(\alpha+2)(\alpha+3) \\
+\beta & (\alpha+2)(\alpha+3) \\
+\gamma & (\alpha+3) \\
+2 \delta &
\end{array}\right\} .
\end{aligned}
$$

$3^{\circ}$. For the term in $\kappa$, writing $r m=0, D=1$, and observing that $[\kappa]^{1},[\kappa]^{2},[\kappa]^{3},[\kappa]^{4}$ give respectively the terms $\kappa,-\kappa, 2 \kappa,-6 \kappa$, this is

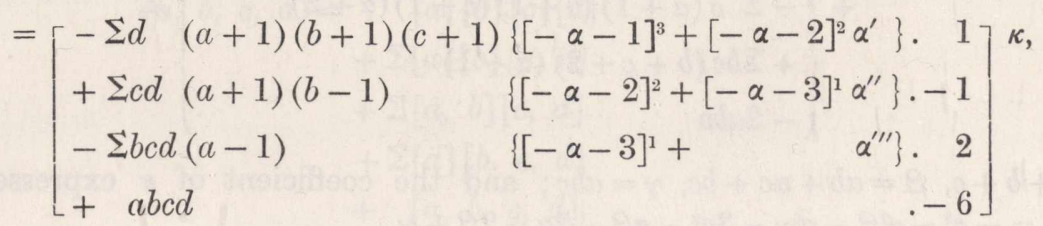


where the terms in \{\} are

that is,

$$
-\left(\alpha+1-\alpha^{\prime}\right)(\alpha+2)(\alpha+3), \quad\left(\alpha+2-\alpha^{\prime \prime}\right)(\alpha+3) \text { and }-\left(\alpha+3-\alpha^{\prime \prime \prime}\right),
$$$$
-(d+1)(\alpha+2)(\alpha+3), \quad(c+d+2)(\alpha+3) \text { and }-(b+c+d+3)
$$

respectively; whence the whole expression is

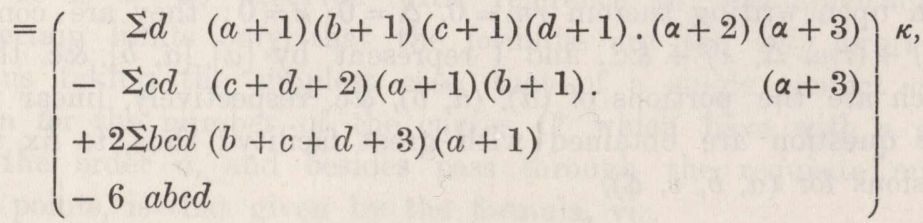

the expression multiplying $(\alpha+2)(\alpha+3)$ is

$$
(a+1)(b+1)(c+1)(d+1) \Sigma d,=(a+1)(b+1)(c+1)(d+1) \alpha
$$

and we have moreover

$$
(a+1)(b+1)(c+1)(d+1)=(1+\alpha+\beta+\gamma+\delta)
$$

the other lines are of course expressible in terms of $(\alpha, \beta, \gamma, \delta)$, but as the law of their formation would then be hidden, I abstain from completing the reduction.

82. The series of formulæ is

$$
\begin{aligned}
& \text { [a] }=(a+1) \mathrm{rm} \\
& +(a+1) a \Delta \\
& \text { - } a \kappa \text {, } \\
& {[a, b]=-(a+1)(b+1)(\alpha+1) \quad \ldots r m} \\
& -(a+1)(b+1)\left\{\begin{array}{r}
\alpha(\alpha+1) \\
-
\end{array}\right\} \Delta \\
& +\left\{\begin{array}{l}
\Sigma b(a+1)(b+1) \\
-\quad a b
\end{array}\right\} \kappa,
\end{aligned}
$$

where $\alpha=a+b, \beta=a b$; and coeff. of $\kappa$ expressed in terms of $\alpha, \beta$ is $=\alpha(1+\alpha+\beta)-\beta$.

$$
\begin{aligned}
{[a, b, c]=} & (a+1)(b+1)(c+1)(\alpha+1)(\alpha+2) \quad \ldots r m \\
& +(a+1)(b+1)(c+1)\left\{\begin{array}{r}
\alpha(\alpha+1)(\alpha+2) \\
-\quad \beta(\alpha+2) \\
-
\end{array}\right\}^{\Delta} \\
& +\left\{\begin{array}{l}
-\Sigma c(a+1)(b+1)(c+1)(\alpha+2) \\
+\Sigma b c(b+c+2)(a+1) \\
-2 a b c
\end{array}\right\}^{\kappa,}
\end{aligned}
$$

where $\alpha=a+b+c, \beta=a b+a c+b c, \gamma=a b c$; and the coefficient of $\kappa$ expressed in terms of $\alpha, \beta, \gamma$ is $=-\alpha^{3}-\alpha^{2} \beta-\alpha^{2} \gamma-3 \alpha^{2}-\alpha \beta-2 \alpha+2 \beta+\gamma$. 


$$
\begin{aligned}
& {[a, b, c, d]=-(a+1)(b+1)(c+1)(d+1)(\alpha+1)(\alpha+2)(\alpha+3) \quad \ldots r m} \\
& -(a+1)(b+1)(c+1)(d+1)(\alpha(\alpha+1)(\alpha+2)(\alpha+3)) \Delta \\
& \left\{\begin{array}{rr}
-\beta & (\alpha+2)(\alpha+3) \\
-\gamma & (\alpha+3) \\
-2 \delta &
\end{array}\right\}
\end{aligned}
$$

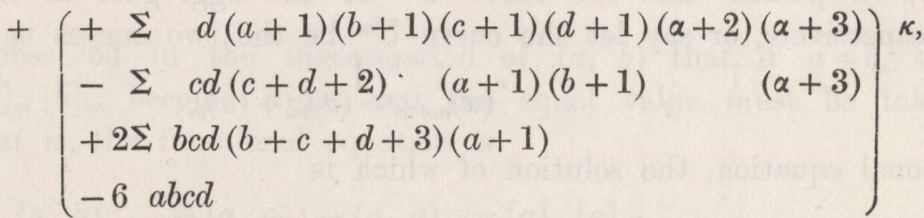

where $\alpha=a+b+c+d, \ldots \delta=a b c d$.

$$
\begin{aligned}
& {[a, b, c, d, e]=(a+1)(b+1)(c+1)(d+1)(e+1)(\alpha+1)(\alpha+2)(\alpha+3)(\alpha+4) \quad \ldots r m} \\
& +(a+1)(b+1)(c+1)(d+1)(e+1)(\alpha(\alpha+1)(\alpha+2)(\alpha+3)(\alpha+4)) \Delta \\
& -\beta \quad(\alpha+2)(\alpha+3)(\alpha+4) \\
& -\gamma \quad(\alpha+3)(\alpha+4) \\
& -2 \delta \\
& (\alpha+4) \\
& -6 \epsilon \\
& +\left(\begin{array}{llr}
-\Sigma e & (a+1)(b+1)(c+1)(d+1)(e+1)(\alpha+2)(\alpha+3)(\alpha+4) \\
+\Sigma d e & (d+e+2)(a+1)(b+1)(c+1) & (\alpha+3)(\alpha+4) \\
-2 \Sigma c d e & (c+d+e+3)(a+1)(b+1) & (\alpha+4) \\
+6 \Sigma b c d e & (b+c+d+e+4)(a+1) \\
-24 a b c d e &
\end{array}\right)
\end{aligned}
$$

where $\alpha=a+b+c+d+e, \beta=\& c ., \ldots \epsilon=a b c d e$.

83. The complete functions $(a),(a, b),(a, b, c)$, \&c. may be expressed by means of the linear terms $[a],[a, b],[a, b, c]$, \&c. as follows, viz. we have

$$
\begin{aligned}
(a)= & {[a], } \\
(a, b)= & {[a][b] } \\
& +[a, b] \\
(a, b, c)= & {[a][b][c] } \\
& +[a][b, c]+[b][a, c]+[c][a, b] \\
& +[a, b, c], \\
(a, b, c, d)= & {[a][b][c][d] } \\
& +\Sigma[a][b][c, d] \\
& +\Sigma[a, b][c, d] \\
& +\Sigma[a][b, c, d] \\
& +[a, b, c, d],
\end{aligned}
$$

C. VI. 
and so on: this is easily verified for $(a, b)$, and without much difficulty for $(a, b, c)$, but in the succeeding cases the actual verification would be very laborious.

84. The theoretical foundation is as follows. Writing for greater distinctness $(a)_{m}$ in place of $(a)$, we have $(a)_{n}$ to denote the number of the curves $C^{r}$ which have with a given curve $U^{m}$ a contact of the order $a$, and which besides pass through $\frac{1}{2} r(r+3)-a$ points. Let the curve $U^{m}$ be the aggregate of two curves of the orders $m, m^{\prime}$ respectively, or say let the curve $U^{m}$ be the two curves $m, m^{\prime}$, then we have

$$
(a)_{m+m^{\prime}}=(a)_{m}+(a)_{m^{\prime}}
$$

a functional equation, the solution of which is

$$
(a)_{m}=[a]_{m},
$$

where $[a]_{m}$ is a linear function of $n, m, \kappa$, or, what is the same thing, of $m, \Delta, \kappa$. I assume for the moment that when the coefficients are determined $[a]_{m}$ would be found to have the value $=[a]$.

Similarly, if $(a, b)_{m}$ denote the number of the curves $C^{r}$ which have with the given curve $U^{m}$ contacts of the orders $a$ and $b$ respectively, and which besides pass through $\frac{1}{2} r(r+3)-a-b$ points, then if the given curve break up into the curves $m, m^{\prime}$, then we have

$$
(a, b)_{m+m^{\prime}}-(a, b)_{m}-(a, b)_{m^{\prime}}=\left\{(a)_{m}(b)_{m^{\prime}}\right\}+\left\{(a)_{m^{\prime}}(b)_{m}\right\},
$$

where $\left\{(a)_{m}(b)_{m^{\prime}}\right\}$ is the number of the curves $C^{r}$ which have with $m$ a contact of the order $a$ and with $m^{\prime}$ a contact of the order $b$, and which pass through the $\frac{1}{2} r(r+3)-a-b$ points; and the like for $\left\{(a)_{m^{\prime}}(b)_{m}\right\}$. Then, not universally, but for values of $a$ and $b$ which are not too great, the order of the aggregate condition is equal to the product of the orders of the component conditious (ante, No. 12), that is, we have

$$
\begin{aligned}
& \left\{(a)_{m}(b)_{m^{\prime}}\right\}=(a)_{m} \cdot(b)_{m^{\prime}}=[a]_{m}[b]_{m^{\prime}}, \\
& \left\{(a)_{m^{\prime}}(b)_{m}\right\}=(a)_{m^{\prime}} \cdot(b)_{m}=[a]_{m^{\prime}}[b]_{m},
\end{aligned}
$$

and thence the functional equation

$$
(a, b)_{m+m^{\prime}}-(a, b)_{m}-(a, b)_{m^{\prime}}=[a]_{m}[b]_{n^{\prime}}+[a]_{n^{\prime}}[b]_{m} .
$$

But $[a]_{m}$, \&c. being linear functions of $m, \Delta, \kappa$, we have

$$
[a]_{m+m^{\prime}}=[a]_{m}+[a]_{m^{\prime}},[b]_{m+m^{\prime}}=[b]_{m}+[b]_{m^{\prime}},
$$

and thence a particular solution of the equation is at once seen to be $[a]_{m}[b]_{m}$; the general solution is therefore

$$
(a, b)_{m}=[a]_{m}[b]_{m}+[a, b]_{m},
$$

where $[a, b]_{m}$ is an arbitrary linear function of $m, \Delta, \kappa$. Hence, assuming for the present that if determined its value would be found to be $=[a, b]$, we have the required formula $(a, b)=[a][b]+[a, b]$. 
The investigation of the expression for $(a, b, c)_{m}$ depends in like manner on the assumption that we have

$$
\left\{(a)_{m}(b, c)_{m^{\prime}}\right\}=(a)_{m} \cdot(b, c)_{m^{\prime}}=[a]_{m}\left\{[b]_{m^{\prime}}[c]_{m^{\prime}}+[b, c]_{m^{\prime}}\right\},
$$

and so in the succeeding cases; and we thus, within the limits in which these assumptions are correct, obtain the series of formulæ for $(a, b),(a, b, c) \ldots$

85. It is to be observed in the investigation of $(a, b)$ that if $a=b$, the two terms $[a]_{m}[b]_{m^{\prime}}$ and $[a]_{m^{\prime}}[b]_{m}$ become equal, and the equal value must be taken not twice but only once, that is, the functional equation is

$$
(a, a)_{m+m^{\prime}}-(a, a)_{m}-(a, a)_{m^{\prime}}=[a]_{m}[a]_{m^{\prime}},
$$

and the solution, writing $\frac{1}{2}[a, a]_{m}$ for the arbitrary linear function, is

$$
(a, a)_{m}=\frac{1}{2}[a]_{m}[a]_{m}+\frac{1}{2}[a, a]_{m},
$$

in which solution it would appear, by the determination of the arbitrary function, that $[a, a]$ has the value obtained from $[a, b]$ by writing therein $b=a$. Writing the equation in the form

$$
(a, a)=\frac{1}{2}[a][a]+\frac{1}{2}[a, a],
$$

and comparing with the equation for $(a, b)$, we see that $[a, b]$ is not to be considered as acquiring any divisor when $b$ is put $=a$, but that the divisor is introduced as a divisor of the whole right-hand side of the equation in virtue of the remark as to the divisor of the functions $(a, b),(a, b, c) \ldots$ in the case of any equalities between the numbers $(a, b, c \ldots)$. This is generally the case, and the foregoing expressions for $[a, b],[a, b, c]$, \&c. are thus to be regarded as true without modification even in the case of any equalities among the numbers $a, b, c \ldots$.

86. To complete according to the foregoing method the determination of the expressions for $(a),(a, b), \ldots$, we have to determine the linear functions $[a],[a, b]$, \&c., which are each of them of the form $f m+g \Delta+h \kappa$, where $(f, g, h)$ are functions of $r$ and of $a, b$, \&c.; and I observe that the determination can be effected if we know the values of $(a),(a, b)$, \&c. in the cases of a unicursal curve without cusps and with a single cusp respectively. Thus assume that in these two cases respectively we have

$$
\begin{aligned}
& (a)=(a+1)(r m-a), \\
& (a)=(a+1)(r m-a)-a .
\end{aligned}
$$

Writing first $\Delta=-1, \kappa=0$, and secondly $\Delta=-1, \kappa=1$, we have

whence

$$
\begin{aligned}
& (a+1)(r m-a)=f m-g, \\
& (a+1)(r m-a)-a=f m-g+h,
\end{aligned}
$$

$$
f=(a+1) r, \quad g=(a+1) a, \quad h=-a,
$$


giving the foregoing value

$$
[a]=(a+1) r m+(a+1) a \Delta-a \kappa .
$$

Similarly, for two contacts assume that we have in the two cases respectively

$$
\begin{aligned}
& (a, b)=(a+1)(b+1)[r m-a-b]^{2}, \\
& (a, b)=(a+1)(b+1)[r m-a-b]^{2}-\{a(b+1)+b(a-1)\}[r m-a-b-1]^{1} .
\end{aligned}
$$

Starting here from the formula $[a, b]=(a, b)-[a][b]=f m+g \Delta+h \kappa$, and writing successively $\Delta=-1, \kappa=0$, and $\Delta=-1, \kappa=1$, we have

$$
\begin{gathered}
(a+1)(b+1)[r m-a-b]^{2}-\{(a+1)(r m-a)\}\{(b+1)(r m-b)\}=f m-g \\
(a+1)(b+1)[r m-a-b]^{2}-\{a(b+1)+b(a+1)\}[r m-a-b-1]^{1} \\
-\{(a+1)(r m-a)-a\}\{(b+1)(r m-b)-b\}=f m-g+h
\end{gathered}
$$

the first of which, putting therein $a+b=\alpha, a b=\beta$, is at once reduced to

$$
(a+1)(b+1)\{r m(-\alpha-1)+\alpha(\alpha+1)-\beta\}=f m-g,
$$

whence $f=-(a+1)(b+1)(\alpha+1) r, g=-(\alpha+1)(b+1)(\alpha(\alpha+1)-\beta)$. And taking the difference of the two equations, we hav 2

$$
\begin{aligned}
- & \{a(b+1)+b(a+1)\}(r m-a-b-1) \\
& +a(b+1)(r m-b)+b(a+1)(r m-a)-a b=h
\end{aligned}
$$

that is $h=(a+1)(b+1)(a+b)-a b$; whence $[a, b]$-has the value above assigned to it.

87. The actual calculation of $[a, b, c]$ would be laborious, and that of the subsequent terms still more so; but it is clear that the principle applies, and that the foregoing values, assuming them to be correct, would be obtained if only we know, for a unicursal curve without cusps, that

$$
(a, b, c, \ldots)=(a+1)(b+1)(c+1) \ldots[r m-(a+b+c, \ldots)]^{t}
$$

( $t$ the number of contacts $a, b, c, \ldots)$, and for a unicursal curve with a single cusp, that

$$
\begin{aligned}
(a, b, c, . .)= & (a+1)(b+1)(c+1) \ldots[r m-(a+b+c \ldots)]^{t} \\
& -\Sigma a(b+1)(c+1) \ldots[r m-(a+b+c \ldots)-1]^{t-1}
\end{aligned}
$$

viz. that the diminution of $(a, b, c, \ldots)$ occasioned by the single cusp is

$$
=[r m-(a+b+c, \ldots)-1]^{t-1} \cdot \Sigma\{a(b+1)(c+1) \ldots\} .
$$


88. Consider a unicursal curve $U^{m}$, and a curve $C^{r}$ having therewith $t$ contacts of the orders $a, b, c, \ldots$ respectively. The coordinates $(x, y, z)$ of any point of the unicursal curve are given as functions of the order $m$ of a variable parameter $\theta$; and substituting these values in the equation of the curve $C^{r}$, we have an equation of the degree $r m$ in $\theta$, but containing the coefficients of $C^{r}$ linearly; this equation gives of course the values of $\theta$ which correspond to the $r m$ intersections of the two curves. Hence in order that the curve $C^{r}$ may have the prescribed contacts with $U^{m}$, the equation of the degree $r m$ in $\theta$ must have $t$ systems of equal roots, viz. a system of $a$ equal roots, another system of $b$ equal roots, \&c.: this implies between the coefficients of the equation an $(a+b+c \ldots)$ fold relation, which may be shown to be of the order $(a+1)(b+1)(c+1) \ldots[r m-(a+b+c \ldots)]^{t}$; and since the coefficients in question are linear in regard to the coefficients in the equation of the curve $C^{r}$, the order of the relation between the last-mentioned coefficients has the same value; that is, the number of the curves $C^{r}$ which have the prescribed contacts with the unicursal curve $U^{m}$ and besides pass through the requisite number of given points, is $=(a+1)(b+1)(c+1) \ldots[r m-(a+b+c \ldots)]^{t}$.

89. The reduction in the case of a cusp appears to be caused as follows:Consider on the curve $U^{m} a$ points indefinitely near to the cusp, and let the condition of the curve $C^{r}$ having the contact of the $a$-th order be replaced by the condition of passing through the $a$ points; that is, consider the curves $C^{r}$ which have with the curve $U^{m}(t-1)$ contacts of the orders $b, c, \ldots$ respectively, which pass through the $a$ points on the curve $U^{m}$ in the neighbourhood of the cusp, and which also pass through the requisite number of arbitrary points. The number of these curves is $=(b+1)(c+1) \ldots[r m-a-(b+c+. .)]^{t-1}$ (the term $r m-a$ instead of $r m$, on account of the given a points on the curve: compare herewith De Jonquières' formula containing $r m-p$ ). Each of these curves, in that it passes through $a$ points in the neighbourhood of the cusp, will ipso facto pass through $a+1$ points (viz. a curve which simply passes through the cusp of a cuspidal curve meets the cuspidal curve there in two points, a curve which touches the cuspidal tangent meets the curve in three points, \&c.), and be consequently, in an improper sense, a curve having a contact of the $a$-th order with the given curve $U^{m}$. I assume that it counts as such curve $a$ times, and this being so, we have, on account of the curves in question, a reduction $=a(b+1)(c+1) \ldots[r m-(a+b+c \ldots)]^{t-1}$. We have in like manner for the curves passing through $b$ points in the neighbourhood of the cusp a reduction $=b(a+1)(c+1) \ldots$ $[r m-(a+b+c \ldots)]^{t-1}$, \&c., and hence when the given unicursal curve $U^{m}$ has a cusp, the total reduction on account of the cusp in the number of the curves $C^{r}$ which have with the given curve the $t$ contacts of the orders $a, b, c, \ldots$ and besides pass through the requisite number of given points, is

$$
=\left\{a(b+1)(c+1) \ldots+b(a+1)(c+1)+\& c_{.}\right\}[r m-(a+b+c \ldots)]^{t-1},
$$

which is the auxiliary theorem in question; some of the steps require however to be more completely made out.

90. I have calculated the following numerical results, wherein, as before, $\Delta=D-1=-m+\frac{1}{2} n+\frac{1}{2} \kappa$. I find also their values in the case where the curve $C^{\prime}$ 
is a conic (that is $r=2$ ) first in terms of $m, n, \kappa$, and finally in terms of $m, n, \alpha$ $(\alpha=3 n+\kappa$, as above). The results are

for $r=2$, that is, curve $C^{r}$ a conic.

\begin{tabular}{|c|c|c|c|c|c|c|c|c|c|c|}
\hline$[1]=$ & $2 r m+$ & $2 \Delta-$ & $\kappa$ & $=$ & $2 m+$ & $n$ & & $=$ & $2 m+$ & $n$ \\
\hline$[2]=$ & $3 r m+$ & $6 \Delta-$ & $2 \kappa$ & $=$ & & $3 n+$ & $\kappa$ & $=$ & & \\
\hline$[3]=$ & $4 r m+$ & $12 \Delta-$ & $3 \kappa$ & $=-$ & $4 m+$ & $6 n+$ & $3 \kappa$ & $=-$ & $4 m-$ & $3 n+$ \\
\hline$[4]=$ & $5 r m+$ & $20 \Delta-$ & $4 \kappa$ & $=-$ & $10 m+$ & $10 n+$ & $6 \kappa$ & $=-$ & $10 m-$ & $8 n+$ \\
\hline
\end{tabular}

$[5]=6 r m+30 \Delta-5 \kappa=-18 m+15 n+10 \kappa=-18 m-15 n+10 a$

$[1,1]=-12 r m-20 \Delta+7 \kappa=-4 m-10 n-3 \kappa=-4 m-\quad n-\quad 3 a$

$[1,2]=-24 r m-60 \Delta+16 \kappa=12 m-30 n-14 \kappa=12 m+12 n-14 a$

$[1,3]=-40 r m-136 \Delta+29 \kappa=56 m-68 n-39 \kappa=56 m+49 n-39 a$

$[1,4]=-60 r m-260 \Delta+46 \kappa=140 m-130 n-84 \kappa \mid=140 m+122 n-84 a$

$[2,2]=-45 r m-144 \Delta+32 \kappa=54 m-72 n-40 \kappa=54 m+48 n-40 a$

$[2,3]=-72 m-288 \Delta+54 \kappa=144 m-144 n-90 \kappa=144 m+126 n-90 \alpha$

$[1,1,1]=160 r m+352 \Delta-98 \kappa=-32 m+176 n+78 \kappa=-32 m-58 n+78 a$

$[1,1,2]=360 r m+1056 \Delta-240 \kappa=-336 m+528 n+288 \kappa=-336 m-336 n+288 a$

$[1,1,3]=672 r m+2528 \Delta-478 \kappa=-1184 m+1264 n+786 \kappa=-1184 m-1094 n+786 a$

$[1,2,2]=756 r m+2700 \Delta+530 \kappa=-1188 m+1350 n+820 \kappa=-1188 m-1110 n+820 a$

$[1,1,1,1]=-3360 m-8928 \Delta+2106 \kappa=2208 m-4464 n-2358 \kappa=2208 m+2610 n-2358 a$

$[1,1,1,2]=-8064 \mathrm{rm}-26784 \Delta+5376 \kappa=10656 m-13392 n-8016 \kappa=10656 m+10656 n-8016 a$

$[1,1,1,1,1]=96768 r m+296448 \Delta-61464 \kappa=-102912 m+148224 n+86760 \kappa=-10912 m-112056 n+86760 \alpha$

(It may be noticed as a curious circumstance that in the last column in the expressions of $[2],[1,2],[1,1,2]$ and $[1,1,1,2]$ respectively, the coefficients of $m$ and $n$ are in each case equal.)

91. In the case of the conic, (1), (2), \&c. are the expressions denoted in the former part of this Memoir by $(1::),(2 \therefore)$, \&c., the number of points being in each case such as to make in all five conditions; calculating these functions by means of the formulæ $(a)=[a]$, \&c., the comparison of the resulting values with the values previously obtained will show $\grave{a}$ posteriori the limits within which the formulæ are applicable; where they cease to be applicable I find the difference, and annex it as a correction to the formula value: I have in some cases given what seems to be the proper theoretical form of this difference. We have
$(1::)$
$=2 m+n$
$(2 \therefore)$
$=\alpha$;
(3:)
$=-4 m-3 n+3 \alpha ;$
(4.)
$=-10 m-8 n+6 \alpha$;
$=-18 m-15 n+10 \alpha-[-3 m+\alpha] \quad(=-[\iota])$; 


$$
\begin{aligned}
2(1,1 \therefore)= & (2 m+n)^{2} \\
- & 4 m-n-3 \alpha ; \\
(1,2:) \quad & (2 m+n) \alpha \\
+ & 12 m+12 n-14 \alpha ; \\
= & (2 m+n)(-4 m-3 n+3 \alpha) \\
(1,3 .) \quad & 56 m+49 n-39 \alpha ; \\
= & (2 m+n)(-10 m-8 n+6 \alpha) \\
(1,4) \quad+ & 140 m+122 n-84 \alpha \\
- & -[(m-3)(-12 m-6 n+6 \alpha)] \quad(=-[(m-3)(4 \iota+2 \kappa)]) ;
\end{aligned}
$$

$2(2,2 \cdot)=\alpha^{2}$

$$
+54 m+48 n-40 \alpha
$$

$(2,3)$

$$
\begin{aligned}
= & \alpha(-4 m-3 n+3 \alpha) \\
& +144 m+126 n-90 \alpha \\
& -[24 m+6 n+(n-12) \alpha] \quad(=-[6 \tau+(n-3) \kappa])
\end{aligned}
$$

$6(1,1,1:)=(2 m+n)^{3}$

$$
\begin{aligned}
& +3(2 m+n)(-4 m-n-3 \alpha) \\
& -32 m-58 n+78 \alpha \\
& -[4 m(m-1)(m-2)] ;
\end{aligned}
$$

$2(2,1,1 \cdot)=(2 m+n)^{2} \alpha$

$$
\begin{aligned}
& +2(2 m+n)(12 m+12 n-14 \alpha)+\alpha(-4 m-n-3 \alpha) \\
& -336 m-336 n+288 \alpha \\
& -[2 \alpha(m-2)(m-3)] \quad(=-[6 n(m-2)(m-3)+2 \kappa(m-2)(m-3)]) ;
\end{aligned}
$$

$2(3,1,1)=(2 m+n)^{2}(-4 m-3 n+3 \alpha)$

$$
\begin{aligned}
& +2(2 m+n)(56 m+49 n-39 x) \\
& +(-4 m-n-3 \alpha)(-4 m-3 n+3 \alpha) \\
& -1184 m-1094 n+786 \alpha
\end{aligned}
$$$$
-\left[\begin{array}{l}
-13 m^{3}-8 m^{2} n+4 m n^{2}+131 m^{2}+92 m n-8 n^{2}-316 m-226 n \\
+\alpha\left(9 m^{2}-87 m-3 n+204\right)
\end{array}\right]
$$

$$
\begin{aligned}
& 2(2,2,1)=(2 m+n) \alpha^{2} \\
& +2 \alpha(12 m+12 n-14 \alpha) \\
& +(2 m+n)(54 m+48 n-40 \alpha) \\
& \left(=-\left[\begin{array}{r}
\delta(2 n+10 m-38) \\
+\kappa\left(3 m^{2}-19 m+30\right) \\
+\iota\left(6 m^{2}-41 m+69\right) \\
+\tau(8 m-32)
\end{array}\right]\right) \\
& -1188 m-1110 n-820 \alpha
\end{aligned}
$$




$$
-\left[\begin{array}{l}
60 m^{2}+42 m n-2.52 m-174 n \\
+\alpha(-40 m+166) \\
+\alpha^{2}(m-4)
\end{array}\right]\left(=-\left[\begin{array}{c}
(m-4)\left(\kappa^{2}-\kappa\right) \\
+6(m-4)(n-3) \kappa \\
+18(m-4) \tau \\
+(m-3)(4 \iota+2 \kappa)
\end{array}\right]\right)
$$

$24(1,1,1,1 \cdot)=(2 m+n)^{4}$

$$
\begin{aligned}
& +6(2 m+n)^{2}(-4 m-n-3 \alpha) \\
& +3(-4 m-n-3 \alpha)^{2} \\
& +4(2 m+n)(-32 m-58 n+78 \alpha) \\
& +2208 m+2610 n-2358 \alpha \\
& -\left[\begin{array}{c}
14 m(m-1)(m-2)(m-3) \\
+ \\
+16 n(m+2)(m-2)(m-3) \\
-36 \alpha(m-2)(m-3)
\end{array}\right] \\
& \left(=-\left[(m-2)(m-3)\left(14 m^{2}+16 m n-14 m-76 n-36 \kappa\right)\right]\right)
\end{aligned}
$$

$6(2,1,1,1)=(2 m+n)^{3} \alpha$

$$
\begin{aligned}
& +3(2 m+n)^{2}(12 m+12 n-14 \alpha) \\
& +3(2 m+n) \alpha(-4 m-n-3 \alpha) \\
& +3(2 m+n)(-336 m-336 n+288 \alpha) \\
& +\alpha(-32 m-58 n+78 \alpha) \\
& +3(-4 m-n-3 \alpha)(12 m+12 n-14 \alpha) \\
& +10656 m+10656 n-8016 \alpha- \\
& -\left[\begin{array}{c}
108 m^{3}+108 m^{2} n-1116 m^{2}-1116 m n+2736 m+2736 n \\
+a\left(7 m^{3}+6 m^{2} n-147 m^{2}-30 m n+1040 m+24 n-2256\right) \\
+\alpha^{2}(-9 m+36),
\end{array}\right]
\end{aligned}
$$

where the correction is

$$
\begin{aligned}
& =-(m-4)\left(\begin{array}{c}
108 m^{2}+108 m n-684 m-684 n \\
+\alpha\left(7 m^{2}-119 m+564+6 n(m-1)\right) \\
-9 \alpha^{2}
\end{array}\right) \\
& =-(m-4)\left(\begin{array}{l}
2 \delta(21 n-36) \\
+2 \tau(18 m-126) \\
+\kappa\left(7 m^{2}+6 m n-65 m-13 n+165\right)-9\left(\kappa^{2}-\kappa\right) \\
+\iota(+16 n-96)
\end{array}\right) ;
\end{aligned}
$$




$$
\begin{aligned}
120(1,1,1,1,1)= & (2 m+n)^{5} \\
+ & 10(2 m+n)^{3}(-4 m-n-3 \alpha) \\
+ & 10(2 m+n)^{2}(-32 m-58 n+78 \alpha) \\
+ & 10(-4 m-n-3 \alpha)(-32 m-58 n+78 \alpha) \\
+ & 5(2 m+n)(2208 m+2610 n-2358 \alpha) \\
+ & -15(2 m+n)(-4 m-n-3 \alpha)^{2} \\
& -\left(\begin{array}{c}
102912 m-112056 n+86760 \alpha \\
-\begin{array}{c}
31 m^{5}+70 m^{4} n+40 m^{3} n^{2} \\
-\quad 460 m^{3} n-120 m^{2} n^{2} \\
235 m^{3}-1030 m^{2} n-400 m n^{2} \\
+ \\
+
\end{array} \\
+\left(\begin{array}{c}
-\quad 210 m^{3}-180 m^{2} n \\
+2970 m^{2}+900 m n \\
-15630 m-720 n \\
+28440
\end{array}\right. \\
+\alpha^{2}(135 m-540),
\end{array}\right)
\end{aligned}
$$

where the correction is

$$
=-(m-4)\left(\begin{array}{c}
31 m^{4}-186 m^{3}-979 m^{2}+6774 m \\
+n\left(70 m^{3}-180 m^{2}-1750 m+9060\right) \\
+40 n^{2}(m+3)(m-2) \\
+\alpha\left(\begin{array}{l}
-210 m^{2}+2130 m-7110 \\
-180 n(m-1)
\end{array}\right) \\
+\alpha^{2} .135
\end{array}\right)
$$

which is

$$
=-(m-4)\left(\begin{array}{l}
31(2 \delta+3 \kappa)^{2}+110(2 \delta+3 \kappa)(2 \tau+3 \iota) \\
+\left(\frac{70}{3} m^{3}+1142 m+3174\right)(\kappa-\iota) \\
+(-14 m-638 n-1524)(2 \delta+3 \kappa) \\
+(-390 m+110 n+4272)(2 \tau+3 \iota) \\
+\left(-210 m^{2}-180 m n+2130 m+990 n-7110\right) \kappa+135 \kappa^{2}
\end{array}\right)
$$

but I have not sought. to further reduce this expression, not knowing the proper form in which to present it.

92. The question which ought now to be considered is to determine the corrections or supplements which should be applied to the foregoing expressions $(a),(a, b)$, \&c., or to their equivalents $[a],[a][b]+[a, b]$, \&c. in order to obtain formulæ for the cases C. VI. 
beyond the limits within which the present formulæ are applicable; but this I am not in a position to enter upon. If the extended formulæ were obtained, it would of course be an interesting verification or application of them to deduce from them the complete series of expressions $(1::),(2 \therefore) \ldots(1,1,1,1,1)$ for the number of the conics which satisfy given conditions of contact with a given curve, and besides pass through the requisite number of given points. It will be recollected that throughout these last investigations, I have put De Jonquières' $p=0$; that is, I have not considered the case of the curves $C^{r}$ which (among the conditions satisfied by them) have with the curve $U^{m}$ contacts of given orders at given points of the curve; it is probable that the general formulæ containing the number $p$ admit of extensions and transformations analogous to the formulæ in which $p$ is put $=0$, but this is a question which $\mathrm{I}$ have not considered.

93. The set of equations $(a)=[a],(a, b)=[a][b]+[a, b]$, \&c., considered irrespectively of the meaning of the symbols contained therein, gives rise to an analytical question which is considered in Annex No. 7.

The question of the conics satisfying given conditions of contact is considered from a different point of view in my Second Memoir above referred to.

\section{Annex No. 1 (referred to in the notice of De Jonquiteres' memoir of 1861).-On the} form of the equation of the curves of a series of given index.

To obtain the general form of the equation of the curves $C^{n}$ of a series of the index $N$, it is to be observed that the equation of any such curve is always included in an equation of the order $n$ in the coordinates, containing linearly and homogeneously certain parameters $a, b, c, \ldots$; this is universally the case, as we may, if we please, take the parameters $(a, b, c, .$.$) to be the coefficients of the general equation of the order n$; but it is convenient to make use of any linear relations between these coefficients so as to reduce as far as possible the number of the parameters. Assume that the number of the parameters is $=\omega+1$, then in order that the curve should form a series (that is, satisfy $\frac{1}{2} n(n+3)-1$ conditions), we must have a $(\omega-1)$ fold relation between the parameters, or, what is the same thing, taking the parameters to be the coordinates of a point in $\omega$-dimensional space, say the parametric point, the point in question must be situate on a $(\omega-1)$ fold locus. Moreover, the condition that the curve shall pass through a given point establishes between the parameters a linear relation (viz. that expressed by the original equation of the curve regarding the coordinates therein as belonging to the given point, and therefore as constants); that is, when the curve passes through a given point, the corresponding positions of the parametric point are given as the intersections of the $(\omega-1)$ fold locus by an omal onefold locus; the number of the curves is therefore equal to the number of these intersections, that is, to the order of the $(\omega-1)$ fold locus; or the index of the series being assumed to be $=N$, the order of the $(\omega-1)$ fold locus must be also $=N$. That is, the general form of the equation of the curves $C^{n}$ which form a series of the index $N$, is that of an equation 
of the order $n$ containing linearly and homogeneously the $\overline{\omega+1}$ coordinates of a certain $(\omega-1)$ fold locus of the order $N$. It is only in a particular case, viz. that in which the $(\omega-1)$ fold locus is unicursal, that the coordinates of a point of this locus can be expressed as rational and integral functions of the order $N$ of a variable parameter $\theta$; and consequently only in this same case that the equation of the curves $C^{n}$ of the series of the index $N$ can be expressed by an equation $(* \gamma x, y, z)^{n}=0$, or $\left(* \gamma(x, y, 1)^{n}=0\right.$, rational and integral of the degree $N$ in regard to a variable parameter $\theta$.

If in the general case we regard the coordinates of the parametric point as irrational functions of a variable parameter $\theta$, then rationalising in regard to $\theta$, we obtain an equation rational of the order $N$ in $\theta$, but the order in the coordinates instead of being $=n$, is equal to a multiple of $n$, say $q n$. Such an equation represents not a single curve but $q$ distinct, curves $C^{n}$, and it is to be observed that if we determine the parameter by substituting therein for the coordinates their values at a given point, then to each of the $N$ values of the parameter there corresponds a system of $q$ curves, only one of which passes through the given point, the other $\overline{q-1}$ curves are curves not passing through the given point, and having no proper connexion with the curves which satisfy this condition.

Returning to the proper representation of the series by means of an equation containing the coordinates of the parametric point, say an equation $(* \gamma x, y, 1)^{n}=0$, involving the two coordinates $(x, y)$, it is to be noticed that forming the derived equation and eliminating the coordinates of the parametric point, we obtain an equation rational in the coordinates $(x, y)$, and also rational of the degree $N$ in the differential coefficient $\frac{d y}{d x}$; in fact since the number of curves through any given point $\left(x_{0}, y_{0}\right)$ is $=N$, the differential equation must give this number of directions of passage from the point $\left(x_{0}, y_{0}\right)$ to a consecutive point, that is, it must give this number of values of $\frac{d y}{d x}$, and must consequently be of the order $N$ in this quantity.

Conversely, if a given differential equation rational in $x, y, \frac{d y}{d x}$, and of the degree $N$ in the last-mentioned quantity $\frac{d y}{d x}$, admit of an algebraical general integral, the curves represented by this integral equation may be taken to be irreducible curves, and this being so they will be curves of a certain order $n$ forming a series of the index $N$; whence the general integral (assumed to be algebraical) is given by an equation of the above-mentioned form, viz. an equation rational of a certain order $n$ in the coordinates, and containing linearly and homogeneously the $\omega+1$ coordinates of a variable parametric point situate on an $(\omega-1)$ fold locus. The integral equation expressed in the more usual form of an equation rational of the order $N$ in regard to the parameter or constant of integration, will be in regard to the coordinates of an order equal to a multiple of $n$, say $=q n$, and for any given value of the parameter will represent not a single curve $C^{n}$, but a system of $q$ such curves: the firstmentioned form is, it is clear, the one to be preferred. 
Annex No. 2 (referred to, No. 17).-On the line-pairs which pass through three given points and touch a given conic.

Taking the given points to be the angles of the triangle formed by the lines $(x=0, y=0, z=0)$, we have to find $(f, g, h)$ such that the conic $(0,0,0, f, g, h \gamma x, y, z)^{2}=0$, or, what is the same thing, $f y z+g z x+h x y=0$, shall reduce itself to a line-pair, and shall touch a given conic $(1,1,1, \lambda, \mu, \nu \gamma x, y, z)^{2}=0$. The condition for a line-pair is that one of the quantities $f, g, h$ shall vanish, viz. it is $f g h=0$; the condition for the contact of the two conics is found in the usual manner by equating to zero the discriminant of the function $1-(\lambda+\theta f)^{2}-(\mu+\theta g)^{2}-(\nu+\theta h)^{2}+2(\lambda+\theta f)(\mu+\theta g)(\nu+\theta h)=(a, b, c, d \gamma \theta, 1)^{3}$ suppose; the values of $a, b, c, d$ being

$$
\begin{aligned}
& a=2 f g h, \\
& b=-\frac{1}{3}\left(f^{2}+g^{2}+h^{2}-2 \lambda g h-2 \mu h f-2 \nu f g\right), \\
& c=\frac{2}{3}((\mu \nu-\lambda) f+(\nu \lambda-\mu) g+(\lambda \mu-\nu) h), \\
& d=1-\lambda^{2}-\mu^{2}-\nu^{2}+2 \lambda \mu \nu .
\end{aligned}
$$

Hence considering $(f, g, h)$ as the coordinates of the parametric point, we have the discriminant-locus $a=0$, and the contact-locus

$$
a^{2} d^{2}+4 a c^{3}+4 b^{3} d-3 b^{2} c^{2}-6 a b c d=0
$$

and at the intersection of the two lvci, $a=0, b^{2}\left(4 b d-3 c^{2}\right)=0$, equations breaking up into the system $(a=0, b=0)$ twice, and the system $a=0,4 b d-3 c^{2}=0$; the former of these is

$$
f g h=0, \quad f^{2}+g^{2}+h^{2}-2 \lambda g h-2 \mu h f-2 \nu f g=0,
$$

which expresses that the intersection of the two lines of the line-pair intersect on the given conic; in fact the system is satisfied by $f=0, g^{2}+h^{2}-2 \lambda g h=0$, giving a line-pair $x(h y+g z)=0$, the two lines whereof intersect on the conic $(1,1,1, \lambda, \mu, \nu \gamma x, y, z)^{2}=0$; and similarly, if $g=0$, then $h^{2}+f^{2}-2 \mu h f=0$, or if $h=0$, then $f^{2}+g^{2}-2 \lambda f g=0$. As noticed above this system occurs twice.

The second system is

$$
\begin{array}{r}
f g h=0, \quad \begin{array}{r}
\left(f^{2}+g^{2}+h^{2}-2 \lambda g h-\right. \\
+
\end{array} \quad+\left((\mu \nu-\lambda f-2 \nu f g)\left(1-\lambda^{2}-\mu^{2}-\nu^{2}+2 \lambda \mu \nu\right)\right. \\
+(\nu \lambda-\mu) g+(\mu \lambda-\nu) h)^{2}=0,
\end{array}
$$

or, as the second equation may also be written,

$$
\begin{aligned}
f^{2}\left(1-\mu^{2}\right) & \left(1-\nu^{2}\right)+g^{2}\left(1-\nu^{2}\right)\left(1-\lambda^{2}\right)+h^{2}\left(1-\lambda^{2}\right)\left(1-\mu^{2}\right) \\
+ & 2 g h\left(1-\lambda^{2}\right)(\mu \nu-\lambda)+2 h f\left(1-\mu^{2}\right)(\nu \lambda-\mu)+2 f g\left(1-\nu^{2}\right)(\lambda \mu-\nu)=0,
\end{aligned}
$$

which expresses that a line of the line-pair touches the conic; in fact the system is satisfied by $f=0, g^{2}\left(1-\nu^{2}\right)+h^{2}\left(1-\mu^{2}\right)+2 g h(\mu \nu-\lambda)=0$, viz. we have here the line-pair $x(h y+g z)=0$, in which the line $h y+g z=0$ touches the conic $(1,1,1, \lambda, \mu, \nu \gamma x, y, z)^{2}=0$ and the like if $g=0$, or if $h=0$. This system it has been seen occurs only once. 
Annex No. 3 (referred to, No. 22).-On the conics which pass through two given points and touch a given conic.

Consider the conics which pass through two given points and touch a given conic. We may take $Z=0$ as the equation of the line through the two given points, and then taking the pole of this line in regard to the given conic and joining it with the two given points respectively, the equations of the joining lines may be taken to be $X=0$ and $Y=0$ respectively. This being so, we have for the given points $(X=0, Z=0)$ and $(Y=0, Z=0)$ respectively, and for the given conic

$$
a X^{2}+b Y^{2}+2 h X Y+c Z^{2}=0
$$

and since the required conic is to pass through the two given points its equation will be of the form

$$
w X^{2}+2 x Y Z+2 y Z X+2 z X Y=0,
$$

where $(x, y, z, w)$ are variable parameters which must satisfy a single condition in order that the last-mentioned conic may touch the given conic. The condition is at once seen to be that obtained by making the equation

$$
\begin{aligned}
& (a+\lambda w) b c \\
- & (a+\lambda w)(h+\lambda z)^{2} \\
- & b \lambda^{2} y^{2} \\
- & c \lambda^{2} z^{2} \\
+ & 2 \lambda^{2} x y(h+\lambda z)=0,
\end{aligned}
$$

considered as a cubic equation in $\lambda$, have a pair of equal roots; or if we write

$$
\begin{aligned}
& A=3 c\left(a b-h^{2}\right), \\
& B=\left(a b-h^{2}\right) w-2 c h z, \\
& C=-a x^{2}-b y^{2}-c z^{2}+2 h(x y-z w), \\
& D=3 z(2 x y-w z),
\end{aligned}
$$

then the required condition is

Hence the conic

$$
A^{2} D^{2}+4 A C^{3}+4 B^{3} D-6 A B C D-3 B^{2} C^{2}=0 .
$$

$$
w X^{2}+2 x Y Z+2 y Z X+2 z X Y=0
$$

satisfies the prescribed conditions, if only the parameters $(x, y, z, w)$ satisfy the lastmentioned equation, that is, if $(x, y, z, w)$ are the coordinates of a point on the sextic surface represented by this equation.

The surface has upon it a cuspidal curve the equations whereof are

$$
\left|\begin{array}{lll}
A, & B, & C \\
B, & C, & D
\end{array}\right|=0
$$


this may be considered as the intersection of the quadric surface $A C-B^{2}=0$ and the cubic surface $A D-B C=0$; and the cuspidal curve is consequently a sextic.

The surface has also a nodal curve made up of two conics; to prove this I write for shortness $k=h-\sqrt{a \bar{b}}, k_{1}=h+\sqrt{a b}$; the values of $A, B, C, D$ then are

$$
\begin{aligned}
& A=-3 c k k_{1}, \\
& B=-k k_{1} w-2 c h z \\
& C=-a x^{2}-b y^{2}-c z^{2}+2 h(x y-z w), \\
& D=3 z(2 x y-z w)
\end{aligned}
$$

and it is in the first place to be shown that the surface contains the conic

$$
x: y: z: w=\theta \sqrt{b}: \theta \sqrt{\bar{a}}: 1:-k \theta^{2}+\frac{c}{k}
$$

where $\theta$ is a variable parameter. Substituting these values, we have

$$
\begin{aligned}
& A=-3 c k k_{1}, \\
& B=k^{2} k_{1} \theta^{2}-c(3 h+\sqrt{a b}), \\
& C=2 k k_{1} \theta^{2}-\frac{c}{k}(3 h-\sqrt{a b}), \\
& D=3\left(k_{1} \theta^{2}-\frac{c}{k}\right)
\end{aligned}
$$

and hence

$$
\begin{aligned}
& A D-B C=-2 k\left(k k_{1} \theta^{2}+\frac{2 c \sqrt{a b}}{k}\right)^{2} \\
& A C-B^{2}=-k^{2}\left(k k_{1} \theta^{2}+\frac{2 c \sqrt{a b}}{k}\right)^{2} \\
& B D-C^{2}=-\left(k k_{1} \theta^{2}+\frac{2 c \sqrt{a b}}{k}\right)^{2}
\end{aligned}
$$

values which satisfy identically the equation of the surface written under the form

$$
(A D-B C)^{2}-4\left(A C^{y}-B^{2}\right)\left(B D-C^{2}\right)=0 .
$$

Moreover, proceeding to form the derived equation, and to substitute therein the foregoing values of $(x, y, z, w)$, we have

$$
\partial A: \partial B: \partial C: \partial D=0: k^{2}: 2 k: 3,
$$

and then the derived equation is

$$
\begin{gathered}
(A D-B C)\left(3 A-2 k B-k^{2} C\right) \\
-2\left(A C-B^{2}\right)\left(3 B-4 k C+k^{2} D\right) \\
-2\left(B D-C^{2}\right)\left(2 k A-2 k^{2} B \quad\right)=0
\end{gathered}
$$


that is,

$$
\begin{aligned}
& -k\left(3 A-2 k B-k^{2} C\right) \\
& +k^{2}\left(\quad 3 B-4 k C+k^{2} D\right) \\
& +\quad\left(2 k A-2 k^{2} B\right),
\end{aligned}
$$

or finally

$$
-k\left(A-3 B k+3 k^{2} C-k^{3} D\right)=0,
$$

which is satisfied by the foregoing values of $A, B, C, D$; hence the conic is a nodal curve on the sextic; and by merely changing the sign of one of the radicals $\sqrt{a}, \sqrt{b}$ (and therefore interchanging $k, k_{1}$ ) we obtain another conic which is also a nodal curve on the surface, that is, we have as nodal curves the two conics

$$
\left\{\begin{array}{l}
x: y: z: w=\theta \sqrt{b}: \quad \theta \sqrt{a}: 1:-k \theta^{2}+\frac{c}{k}, \quad \text { and } \\
x: y: z: w=\theta \sqrt{b}:-\theta \sqrt{ } a: 1:-k_{1} \theta^{2}+\frac{c}{k_{1}} .
\end{array}\right.
$$

It is to be remarked that each of the nodal conics meets the cuspidal curve in two points, viz. writing for shortness $\Theta=\frac{1}{k} \sqrt{\frac{-2 c \sqrt{a b}}{k_{1}}}, \Theta_{1}=\frac{1}{k_{1}} \sqrt{\frac{2 c \sqrt{a b}}{k}}$, for the intersections of the first conic we have

$$
x: y: z: w=\Theta \sqrt{a}: \Theta \sqrt{b}: 1: \frac{c}{k_{1}} \text { and }=-\Theta \sqrt{a}:-\Theta \sqrt{b}: 1: \frac{c}{k_{1}}
$$

and for the intersections with the second conic

$$
x: y: z: w=\Theta_{1} \sqrt{a}:-\Theta_{1} \sqrt{b}: 1: \frac{c}{k} \text { and }=-\Theta_{1} \sqrt{a}: \Theta_{1} \sqrt{b}: 1: \frac{c}{k}
$$

The condition of passing through any arbitrary point establishes a linear relation between the parameters $(x, y, z, w)$. Hence, if the conic in addition to the prescribed conditions passes through two other given points, the point $(x, y, z, w)$ is given as the intersection of a line with the sextic surface; the number of intersections is $=6$. If $(x, y, z, w)$ is situate on the cuspidal curve, then the conic instead of simply touching the given conic will have with it a contact of the second order, and if we besides suppose that the conic passes through a given point, then the point $(x, y, z, w)$ is given as the intersection of the cuspidal curve with a plane; the number is $=6$. Similarly, if the conic has two contacts with the given conic, and besides passes through a given point, then the point $(x, y, z, w)$ is given as the intersection of the nodal curve by a plane; the number is $=4$. Finally (observing that in the case in question of the contacts of a conic with a conic we cannot have three simple contacts, or a simple contact and one of the second order), a point of intersection of the nodal and cuspidal curves answers to a contact of the third order; and the number is $=4$. That is, the theory 
of the sextic surface leads to the following values (agreeing with those obtained from the formulæ by writing therein $m=n=2, \alpha=6$ ), viz.

$$
\begin{aligned}
& (1::)=6, \quad=2 m+n, \\
& (1,1 \therefore)=4, \quad=2 m^{2}+2 m n+\frac{1}{2} n^{2}-2 m-\frac{1}{2} n-\frac{3}{2} \alpha, \\
& (2 \therefore)=6, \quad=\alpha \\
& (3:)=4, \quad=-4 m-3 n+3 \alpha .
\end{aligned}
$$

I remark that the section by an arbitrary plane is a sextic curve having 6 cusps and 4 nodes; it is therefore a unicursal sextic; this suggests the theorem that the sextic surface is also unicursal, viz. that the coordinates are expressible rationally in terms of two parameters; I have found that this is in fact the case. In doing this there is no loss of generality in supposing that $a=b=c=1$; and assuming that this is so, and putting also $-1+h=k, 1+h=k_{1}$, and therefore $2 h=k+k_{1}$, we have

$$
\begin{aligned}
& A=-3 k k_{1}, \\
& B=-k k_{1} w-\left(k+k_{1}\right) z, \\
& C=-x^{2}-y^{2}-z^{2}+\left(k+k_{1}\right)(x y-z w), \\
& D=3 z(2 x y-z w) .
\end{aligned}
$$

The equation of the sextic surface being, as before,

$$
A^{2} D^{2}+4 A C^{3}+4 B^{3} D-3 B^{2} C^{2}-6 A B C D=0,
$$

I say that this equation is satisfied on writing therein

$$
\begin{aligned}
& x+y=\sqrt{-\frac{2}{k_{1}}}\left(1-k_{1} \alpha\right) \sin \phi, \\
& x-y=\sqrt{\frac{2}{k}}(1+k \alpha) \cos \phi \\
& z \quad=1 \\
& w \quad=\left(2 \alpha-\frac{1}{k}\right) \cos ^{2} \phi+\left(2 \alpha-\frac{1}{k_{1}}\right) \sin ^{2} \phi,
\end{aligned}
$$

where $(\alpha, \phi)$ are arbitrary. In fact these values give

$$
\begin{array}{rlr}
\frac{1}{3} A & =-k k_{1} \quad \cos ^{2} \phi-k k_{1} \quad \sin ^{2} \phi, \\
B & =-k\left(2 \alpha k_{1}+1\right) \cos ^{2} \phi-k_{1}(2 \alpha k+1) \sin ^{2} \phi, \\
C & =-k\left(\alpha k_{1}+2\right) \cos ^{2} \phi-k_{1} \alpha(\alpha k+2) \sin ^{2} \phi, \\
\frac{1}{3} D & =-k \alpha^{2} \quad \cos ^{2} \phi-k_{1} \alpha^{2} & \sin ^{2} \phi,
\end{array}
$$

whence, $\omega$ being arbitrary, we have

$$
\begin{aligned}
\frac{1}{3}(A, B, C, D \gamma \omega, 1)^{3} \\
\quad=-\left[k \cos ^{2} \phi\left(k_{1} \omega+1\right)+k_{1} \sin ^{2} \phi(k \omega+1)\right](\omega+\alpha)^{2},
\end{aligned}
$$


viz. the equation $(A, B, C, D \gamma \omega, 1)^{3}=0$, considered as a cubic equation in $\omega$, has the twofold root $\omega=-\alpha$, that is, we have the above relation between $(A, B, C, D)$. Whence also writing $\sin \phi=\frac{2 \lambda}{1+\lambda^{2}}, \cos \phi=\frac{1-\lambda^{2}}{1+\lambda^{2}}$, the equation of the surface is satisfied by the values

$$
\begin{aligned}
x+y: x-y: z: w= & \sqrt{-\frac{2}{k_{1}}}\left(1-k_{1} \alpha\right) 2 \lambda\left(1+\lambda^{2}\right) \\
& : \sqrt{\frac{2}{k}}(1+k \alpha) \quad\left(1-\lambda^{4}\right) \\
& : \\
& :\left(2 \alpha-\frac{1}{k}\right)\left(1-\lambda^{2}\right)^{2}+\left(2 \alpha-\frac{1}{k_{1}}\right) 4 \lambda^{2},
\end{aligned}
$$

or the coordinates are expressed rationally in terms of $\alpha, \lambda$.

Annex No. 4 (referred to, Nos. 22 and 71).-On the Conics which touch a cuspidal cubic.

In the cuspidal cubic, if $x=0$ be the equation of the tangent at the cusp, $y=0$ that of the line joining the cusp with the inflexion, and $z=0$ that of the tangent at the cusp, then the equation of the curve is $y^{2}=x^{2} z$; the coordinates of a point on the cubic are given by $x: y: z=1: \theta: \theta^{3}$, where $\theta$ is a variable parameter; and we have, at the cusp $\theta=\infty$, at the inflexion $\theta=0$. In the cubic, $m=n=3, \alpha(=3 n+\kappa)=10$.

Considering now the conic

$$
\left(a, b, c, f, g, h \gamma(x, y, z)^{2}=0,\right.
$$

this meets the cubic in the 6 points the parameters of which are determined by the equation

$$
\left(a, b, c, f, g, h \gamma 1, \theta, \theta^{3}\right)^{2}=0
$$

or, what is the same thing,

$$
(c, 0,2 f, 2 g, b, 2 h, a \gamma \theta, 1)^{6}=0 .
$$

The discriminant of this sextic function contains the factor $c$, hence equating the residual factor to zero, we obtain the equation of the contact-locus in the form

$$
(c, f, g, b, h, a)^{9}=0 .
$$

It follows that the number of the conics $(1::)$ is $=9$, which agrees with the general value $(1::)=2 m+n$. If the conic pass through the cusp we have $c=0$, and the equation in $\theta$ is reduced to a quartic; it is convenient to alter the letters in such wise that the quartic equation may be obtained in the standard form $(a, b, c, d, e \gamma \theta, 1)^{4}=0$; viz. this will be the case if the equation of the conic is taken to be

$$
\left(e, 6 c, 0, \frac{1}{2} a, 2 b, 2 d \gamma(x, y, z)^{2}=0\right. \text {, }
$$

C. VI. 
and we then obtain the equation of the contact-locus in the form

$$
\left(a e-4 b d+3 c^{2}\right)^{3}-27\left(a c e+2 b c d-a d^{2}-b^{2} e-c^{3}\right)^{2}=0
$$

which is a onefold locus of the order 6 . It follows that we have

$$
(1 \kappa 1,1 \therefore)=6 \text {, agreeing with }(\overline{1 \kappa 1}, 1 \therefore)=n+2 m-3 \text {. }
$$

The condition in order that the conic may touch a given line is given by an equation of the form

$$
\left(* \gamma a^{2}, a b, b^{2}, 2 c e-3 d^{2}, a e-8 b d, a d-12 b c\right)^{1}=0,
$$

which is a onefold locus of the order 2 ; it at once follows that we have

$$
(\overline{1 \kappa} 1,1: /)=12 \text {, agreeing with }(1 \kappa 1,1: /)=2 n+4 m-6 .
$$

It is a matter of some difficulty to show that we have

$$
(\overline{1 \kappa 1}, 1 \cdot / /)=18 \text {, agreeing with }(\overline{1 \kappa 1}, 1 \cdot / /)=4 n+4 m-6 \text {; }
$$

but I proceed to effect this, first remarking that I do not attempt to prove the remaining case

$$
(\overline{1 \kappa 1}, 1 / / /)=15 \text {, agreeing with }(1 \kappa 1,1 / / /)=4 n+2 m-3 .
$$

Investigation of the value $(1 \kappa 1,1 \cdot / /)=18$ :

We have the sextic locus

$$
\left(a e-4 b d+3 c^{2}\right)^{3}-27\left(a c e+2 b c d-a d^{2}-b^{2} e-c^{3}\right)^{2}=0,
$$

and combined therewith two quadric loci,

$$
\begin{aligned}
& \left(* \gamma a^{2}, a b, b^{2}, 2 c e-3 d^{2}, a e-8 b \bar{d}, a d-12 b c\right)^{1}=0, \\
& \left(*^{\prime} \gamma a^{2}, a b, b^{2}, 2 c e-3 d^{2}, a e-8 b d, a d-12 b c\right)^{1}=0,
\end{aligned}
$$

which intersect in a threefold locus of the order 24; it is to be shown that this contains as part of itself the quadric threefold locus $\left(a=0, b=0,2 c e-3 d^{2}=0\right)$ taken three times, leaving a residual locus of the order $24-6,=18$.

We may imagine the coordinates $a, b, c, d, e$ expressed as linear functions of any four coordinates, and so reduce the problem from a problem in 4-dimensional space to one in ordinary 3-dimensional space. We have thus a sextic surface, and two quadric surfaces; the sextic is a developable surface or torse, having for one of its generating lines the line $a=0, b=0$, and for the tangent plane along this line the plane $a=0$; the two quadric surfaces meet in a quartic curve passing through the two points $\left(a=0, b=0,3 c e-2 d^{2}=0\right)$, which are points on the torse; it is to be shown that each of these points counts three times among the intersections of the torse with the quartic curve, the number of the remaining intersections being therefore $24-6,=18$; and in order thereto it is to be shown that each of the points in question $\left(a=0, b=0,3 c e-2 d^{2}=0\right)$ 
is situate on the nodal line of the torse, and that the quartic curve touches there the sheet which is not touched by the tangent plane $a=0$; for this being so the quartic curve touching one sheet and simply meeting the other sheet meets the torse in three consecutive points, or the two points of intersection count each of them three times.

The torse has the cuspidal line

$$
S=a e-4 b d+3 c^{2}=0, \quad T=a c e+2 b c d-a d^{2}-b^{2} e-c^{3}=0,
$$

and the nodal line

$$
\left\|\begin{array}{ccccc}
6\left(a c-b^{2}\right), & 3(a d-b c), & a e+2 b d-3 c^{2}, & 3(b e-c d), & 6\left(c e-d^{2}\right) \\
a & b & b & c & d
\end{array}\right\|
$$

and the equations of the nodal line are satisfied by the values $\left(a=0, b=0,3 c e-2 d^{2}=0\right)$ of the coordinates of the points in question. To find the tangent planes at these points, starting from the equation $S^{3}-27 T^{2}=0$ of the torse, taking $(A, B, C, D, E)$ as current coordinates, and writing

$$
\partial=A \partial_{a}+B \partial_{b}+C \partial_{c}+D \partial_{d}+E \partial_{e}
$$

then the equation of the tangent plane is in the first instance given in the form $S^{2} \partial S-18 T \partial T=0$, which writing therein $\left(a=0, b=0,3 c e-2 d^{2}=0\right)$ assumes, as it should do, the form $0=0$; the left-hand side is in fact found to be $9 c^{3}\left(3 c e-2 d^{2}\right) A$. Proceeding to the second derived equation, this is $S^{2} \partial^{2} S+2 S(\partial S)^{2}-18 T \partial^{2} T-18(\partial T)^{2}=0$, or substituting the values of the several terms, the equation is

$$
\begin{aligned}
& 9 c^{4}\left(A E-4 B D+3 C^{2}\right) \\
+ & 3 c^{2}(e A-4 d B+6 c C)^{2} \\
+ & 18 c^{3}\left\{e\left(A C-B^{2}\right)+2 d(B C-A D)+c\left(A E+2 B D-3 C^{2}\right)\right\} \\
- & 9\left\{\left(c e-d^{2}\right) A+2 c d B-3 c^{2} C^{2}=0 ;\right.
\end{aligned}
$$

the terms in $B C, B D, C^{2}$ vanish identically, that in $B^{2}$ is $(48-36=) 12 c^{2} d^{2}-18 c^{3} e$, $=-6 c^{2}\left(3 c e-2 d^{2}\right) B^{2}$, which also vanishes; hence there remain only the terms divisible by $A$, giving first the tangent plane $A=0$, and secondly the other tangent plane,

$$
\begin{aligned}
& A\left(-6 c^{2} e^{2}+18 c d^{2} e-9 d^{4}\right) \\
+ & B\left(-60 c^{2} d e+36 c d^{3}\right) \\
+ & C\left(108 c^{3} e-54 c^{2} d^{2}\right) \\
+ & D\left(-36 c^{3} d\right) \\
+ & E .27 c^{4}=0 .
\end{aligned}
$$

Taking the equations of the quadric surfaces to be

$$
\begin{aligned}
& \left.(\lambda, \mu, \nu, \rho, \sigma, \tau\} a^{2}, b^{2}, a b, 3 c e-2 d^{2}, a e-8 b d, a d-12 b c\right)=0 \text {, } \\
& \left(\lambda^{\prime}, \mu^{\prime}, \nu^{\prime}, \rho^{\prime}, \sigma^{\prime}, \tau^{\prime} \mathcal{X} \quad \text { " }, \quad, \quad\right)=0 \text {, }
\end{aligned}
$$


the equations of the tangent planes are

$$
\begin{aligned}
& \rho(3 c E+3 e C-4 d D)+\sigma(e A-8 d B)+\tau(d A-12 c B)=0, \\
& \rho^{\prime}(\quad)+\sigma^{\prime}(")+\tau^{\prime}(\quad, \quad=0,
\end{aligned}
$$

in all which equations we have $3 c e-2 d^{2}=0$; and if to satisfy this equation we write $c: d: e=2: 3 \beta: 3 \beta^{2}$, then the equations of the tangent planes become

$$
\begin{array}{r}
\beta^{3}(A \beta-8 B)+8\left(3 C \beta^{2}-4 D \beta+2 E\right)=0, \\
\rho\left(3 C B^{2}-4 D \beta+2 E\right)+(\sigma \beta+\tau)(A \beta-8 B)=0, \\
\rho^{\prime}(\quad)+\left(\sigma^{\prime} \beta-\tau\right)(\quad, \quad=0,
\end{array}
$$

or the three tangent planes intersect in the line $A \beta-8 B=0,3 C \beta^{2}-4 D \beta+2 E=0$, which completes the proof.

Reverting to the sextic locus,

$$
\left(a e+4 b d-3 c^{2}\right)^{2}-27\left(a c e+2 b c d-a d^{2}-b^{2} e-c^{3}\right)^{2}=0,
$$

considered as a locus in 4-dimensional space depending on the five corrdinates $(a, b, c, d, e)$, this has upon it the twofold locus

$$
a e-4 b d+3 c^{2}=0, \quad a c e+2 b c d-a d^{2}-b^{2} e-c^{3}=0,
$$

say the cuspidal locus, of the order 6 , and the twofold locus

$$
\mid \begin{array}{ccccc}
6\left(a c-b^{2}\right), & 3(a d-b c), & a e+2 b d-3 c^{2}, & 3(b e-c d), & 6\left(c e-d^{2}\right) \\
a & b & b & c & d
\end{array} \|=0
$$

say the nodal locus, of the order 4 : there is also a threefold locus,

$$
\| \begin{array}{llll}
a, & b, & c, & d \\
b, & c, & d, & e
\end{array} \mid=0
$$

say the supercuspidal locus, of the order 4 . We thence at once infer

$(\overline{1} \overline{1}, 2 \quad:)=6$, agreeing with $(\overline{1 \kappa} \overline{1}, 2 \quad:)=\alpha-4$,

$$
\begin{array}{lllll}
(\overline{1 \kappa 1}, 1,1:)=4, & \# & & (\overline{1 \kappa 1}, 1,1:)=2 m^{2}+2 m n+\frac{1}{2} n^{2}-8 m-\frac{7}{2} n+13-\frac{3}{2} \alpha, \\
(\overline{1 \kappa 1}, 3 \quad:)=4, & , & & (1 \kappa 1,3 \quad:)=-4 m-3 n-5+3 \alpha
\end{array}
$$

but I have not investigated the application to the symbols with $/ /$ or $/ /$.

If the conic, instead of simply passing through the cusp, touches the cuspidal tangent, then in the equation $\left(a, b, 0, f, g, h \gamma(x, y, z)^{2}=0\right.$ of the conic we have $f=0$, or, what is the same thing, in the equation $\left(e, 6 c, 0, \frac{1}{2} a, 2 b, 2 d \gamma x, y, z\right)^{2}=0$ of the conic we have $a=0$. The equation in $\theta$ is thus reduced to $4 b \theta^{3}+6 c \theta^{2}+4 d \theta+e=0$. For the independent discussion of this case it is convenient to alter the coefficients so that the equation in $\theta$ may be in the standard form $(a, b, c, d \gamma \theta, 1)^{3}=0$, viz. we 
assume the equation of the conic to be $\left(d, 3 b, 0,0, \frac{1}{2} a, \frac{3}{2} c \gamma(x, y, z)^{2}=0\right.$. The equation of the contact-locus then is

$$
a^{2} d^{2}+4 a c^{3}+4 b^{3} d-6 a b c d-3 b^{2} c^{2}=0,
$$

viz. this is a developable surface, or torse, of the order 4 , and we at once infer

$$
(\overline{2 \kappa 1}, 1:)=4 \text {, agreeing with }(\overline{2 \kappa 1}, 1:)=2 m+n-5 .
$$

I will show also that we have

and

$$
(\overline{2 \kappa 1}, 1 \cdot /)=6 \text {, agreeing with }(\overline{2 \kappa 1}, 1 \cdot /)=2 m+2 n-6,
$$

$$
(\overline{2 \kappa 1}, 1 / /)=5, \quad \# \quad " \quad(\overline{2 \kappa 1}, 1 / /)=m+2 n-4 .
$$

The condition that the conic may touch an arbitrary line $\alpha x+\beta y+\gamma z=0$, is in fact

$$
\left(0,-\frac{1}{4} a^{2}, \frac{3}{4}\left(4 b d-3 c^{2}\right), \frac{3}{4} a c,-\frac{3}{2} a b, 0 \gamma(\alpha, \beta, \gamma)^{2}=0,\right.
$$

which, considering therein $(a, b, c, d)$ as coordinates, is the equation of a quadric surface passing through the conic $a=0,4 b d-3 c^{2}=0$; the quartic torse also passes through this conic; hence the quadric surface and the torse intersect in this conic, which is of the order 2, and in a residual curve of the order 6 ; and the number of the conics $(2 \kappa 1,1 \cdot /)$ is equal to the order of this residual curve, that is, it is $=6$.

If the conic touch a second arbitrary line $\alpha^{\prime} x+\beta^{\prime} y+\gamma^{\prime} z=0$, then we have in like manner the quadric surface

$$
\left(0,-\frac{1}{4} a^{2}, \frac{3}{4}\left(4 b d-3 c^{2}\right), \frac{3}{4} a c,-\frac{3}{2} a b, 0 \gamma\left(\alpha^{\prime}, \beta^{\prime}, \gamma^{\prime}\right)^{2}=0 ;\right.
$$

that is, we have the quartic torse and two quadric surfaces, each passing through the conic $a=0,4 b d-3 c^{2}=0$, and it is to be shown that the number of intersections not on this conic is $=5$. The two quadric surfaces intersect in the conic and in a second conic; this second conic meets the torse in 8 points, but 2 of these coincide with the point $a=0, b=0, c=0$, which is one of the intersections of the two conics (the point $a=0, b=0, c=0$ is in fact a point on the cuspidal edge of the torse, and, the conic passing through it, reckons for 2 intersections), and 1 of the 8 points coincides with the other of the intersections of the two conics; there remain therefore $8-2-1,=5$ intersections, or we have $(\overline{2 \kappa 1}, 1 / /)=5$.

Annex No. 5 (referred to, Nos. 22 and 71). On the Conics which have contact of the third order with a given cuspidal cubic, and two contacts (double contact) with a given conic.

Let the equation of the cuspidal cubic be $x^{2} z-y^{3}=0 \quad(x=0$ tangent at cusp, $z=0$ tangent at inflexion, $y=0$ line joining cusp and inflexion; equation satisfied by

$$
\left.x: y: z=1: \theta: \theta^{3}\right)
$$

and let the equation of the given conic be

$$
U=\left(a, b, c, f, g, h \chi(x, y, z)^{3}=0 ;\right.
$$


then writing

$$
\begin{aligned}
\Theta & =\left(a, b, c, f, g, h \gamma 1, \theta, \theta^{3}\right)^{2} \\
& =c \theta^{6}+2 f \theta^{4}+2 g \theta^{3}+b \theta^{2}+2 h \theta+c,
\end{aligned}
$$

the equation of a conic having with the given cubic at a given point $\left(1, \theta, \theta^{3}\right)$ contact of the second order, and having double contact with the given conic, is

$$
\left|\begin{array}{cccc}
\sqrt{U}, & x, & y, & z \\
\sqrt{\Theta}, & 1, & \theta, & \theta^{3} \\
(\sqrt{\Theta})^{\prime} & . & 1, & 3 \theta^{2} \\
(\sqrt{\Theta})^{\prime \prime} & . & . & 6 \theta
\end{array}\right|=0
$$

viz. in the rational form this is

$$
36 \theta^{2} U-\left|\begin{array}{cccc} 
& x, & y, & z \\
\sqrt{\Theta}, & 1, & \theta, & \theta^{3}=0, \\
(\sqrt{\Theta})^{\prime} & . & 1, & 3 \theta^{2} \\
(\sqrt{\Theta})^{\prime \prime} & . & . & 6 \theta
\end{array}\right|
$$

and this will have at the point $\left(1, \theta, \theta^{3}\right)$ a contact of the third order if $\theta$ be determined by

viz. this is

$$
\left|\begin{array}{cccc}
\sqrt{\Theta}, & 1, & \theta, & \theta^{3} \\
(\sqrt{\Theta})^{\prime} & \cdot & 1, & 3 \theta^{2} \\
(\sqrt{\Theta})^{\prime \prime} & . & . & 6 \theta \\
(\sqrt{\Theta})^{\prime \prime \prime} & . & . & 6
\end{array}\right|=0
$$

$$
\Theta(\sqrt{\Theta})^{\prime \prime \prime}-(\sqrt{\Theta})^{\prime \prime}=0 ;
$$

or developing and multiplying by $\Theta^{\frac{5}{2}}$, this is

$$
\theta\left\{\Theta^{2} \Theta^{\prime \prime \prime}-\frac{3}{2} \Theta \Theta^{\prime} \Theta^{\prime \prime}+\frac{3}{4} \Theta^{\prime 3}\right\}-\left(\Theta^{2} \Theta^{\prime \prime}-\frac{1}{2} \Theta \Theta^{\prime 2}\right)=0,
$$

or, what is the same thing,

$$
\Theta^{2}\left(\theta \Theta^{\prime \prime \prime}-\Theta^{\prime \prime}\right)+\Theta \Theta^{\prime}\left(-\frac{3}{2} \theta \Theta^{\prime \prime}+\frac{1}{2} \Theta^{\prime}\right)+\Theta^{\prime 2} \cdot \frac{3}{4} \theta \Theta^{\prime}=0 ;
$$

and substituting for $\Theta$ its value, this is

$$
\begin{aligned}
& \left(c \theta^{6}+2 f \theta^{4}+2 g \theta^{3}+b \theta^{2}+2 h \theta+a\right)^{2}\left(45 c \theta^{4}+12 f \theta^{2}-b\right) \\
+ & \left(c \theta^{6}+2 f \theta^{4}+2 g \theta^{3}+b \theta^{2}+2 h \theta+a\right)\left(3 c \theta^{5}+4 f \theta^{3}+3 g \theta^{2}+b \theta+h\right)
\end{aligned}
$$

$$
\left(-42 c \theta^{5}-32 f \theta^{3}-15 g \theta^{2}-2 b \theta+h\right)
$$

$+3 \theta\left(3 c \theta^{5}+4 f \theta^{3}+3 g \theta^{2}+b \theta+h\right)^{3}=0$. 
The coefficients of the powers $16,15,14,13$ of $\theta$ all vanish, so that this is in fact an equation of the twelfth order $(* \gamma \theta, 1)^{12}=0$; and putting, as usual,

$$
\left(b c-f^{2}, c a-g^{2}, a b-h^{2}, g h-a f, h f-b g, f g-c h\right)=(A, B, C, F, G, H),
$$

the equation is found to be

$$
\begin{aligned}
& \text { - } 4 c A \theta^{12} \\
& +30 c H \quad \theta^{11} \\
& \left.\begin{array}{l}
-36 c B \\
+16 f A
\end{array}\right\} \theta^{10} \\
& \left.\begin{array}{l}
-10 c G \\
+40 g A
\end{array}\right\} \theta^{\circ} \\
& +20 b A \\
& -60 f B\} \theta^{8} \\
& \begin{array}{r}
+\quad 72 h A \\
+\quad 9 g B \\
\end{array} \\
& +45 a B \\
& \text { - } 22 b H \\
& -20 f C\} \theta^{4} \\
& +10 h G) \\
& +\quad 40 a \mathrm{~A} \\
& -130 h \mathrm{H} \\
& +10 g G \theta^{\theta^{\circ}} \\
& \left.\begin{array}{l}
+5 h F^{\prime} \\
+20 a G
\end{array}\right\} \theta^{3} \\
& +40 f F \\
& \left.\begin{array}{r}
-4 b C \\
-12 a F^{\prime}
\end{array}\right\} \theta^{2} \\
& +33 h B \\
& +\quad 2 b G \theta^{5} \\
& \text { - } 5 h C \theta \\
& -90 g H
\end{aligned}
$$

where the form of the coefficients may be modified by means of the identical equations

$$
\begin{aligned}
& (A, H, G \gamma a, h, g)=K, \\
& \left(H, B, F^{\gamma}, \quad\right)=0, \\
& (G, F, C \chi \quad)=0, \\
& (A, H, G \gamma h, b, f)=0, \\
& \left(H, B, F^{\gamma} "\right)=K, \\
& (G, F, C \gamma \quad)=0, \\
& (A, H, G \gamma g, f, c)=0, \\
& \left(H, B, F^{\gamma} "\right)=0, \\
& (G, F, C \gamma \quad, \quad)=K .
\end{aligned}
$$

There is consequently a conic answering to each value of $\theta$ given by this equation, or we have in all 12 conics.

In the case where the given conic breaks up into a pair of lines, or say,

$$
(a, b, c, f, g, h \chi x, y, z)^{2}=2(\lambda x+\mu y+\nu z)\left(\lambda^{\prime} x+\mu^{\prime} y+\nu^{\prime} z\right),
$$

then, writing for shortness

we have

$$
\mu \nu^{\prime}-\mu^{\prime} \nu, \nu \lambda^{\prime}-\nu^{\prime} \lambda, \lambda \mu^{\prime}-\lambda^{\prime} \mu=X, Y, Z,
$$

$(A, B, C, F, G, H)=\left(X^{2}, Y^{2}, Z^{2}, Y Z, Z X, X Y\right)$. 
Substituting these values, but retaining $(a, b, c, f, g, h)$ as standing for their values $a=2 \lambda \lambda^{\prime}$, \&c., the equation in $\theta$ is found to contain the cubic factor $2 X \theta^{3}-3 Y \theta^{2}+Z$, where it is to be observed that this factor equated to zero determines the values of $\theta$ which correspond to the points of contact with the cuspidal cubic of the tangents from the point $(X, Y, Z)$, which is the intersection of the lines $\lambda x+\mu y+\nu z=0$, and $\lambda^{\prime} x+\mu^{\prime} y+\nu^{\prime} z=0$; and omitting the cubic factor, the residual equation is found to be

\begin{tabular}{|c|c|c|c|c|c|c|c|c|c|}
\hline $2 c X$ & $-12 c Y$ & $-8 f X$ & $\begin{array}{l}-20 g X \\
-12 f Y \\
+\quad 4 c Z\end{array}$ & $\begin{array}{l}-10 b X \\
+\quad 3 g Y\end{array}$ & $\begin{array}{l}-40 h X \\
-8 b Y\end{array}$ & $\begin{array}{l}-20 a X \\
+17 h Y \\
+\quad 7 g Z\end{array}$ & $\begin{array}{l}+15 a Y \\
+\quad 4 b Z\end{array}$ & $+5 h Z$ & $+a Z$ \\
\hline
\end{tabular}

where the form of the coefficients may be modified by means of the identical equations

$$
\begin{aligned}
& a X+h Y+g Z=0 \\
& h X+b Y+f Z=0 \\
& g X+f Y+c Z=0
\end{aligned}
$$

The equation is of the 9 th order, and there are consequently 9 conics.

Annex No. 6 (referred to, No. 48).-Containing, with the variation referred to in the text, ZEUTHEN's forms for the characteristics of the conics which satisfy four conditions.

$$
\begin{aligned}
& (::)=n+2 m, \\
& (\therefore /)=2 n+4 m, \\
& (: / /)=4 n+4 m, \\
& (\cdot / / /)=4 n+2 m, \\
& (/ / /)=2 n+m ;
\end{aligned}
$$

$(1,1)$

$$
\begin{aligned}
& (\therefore)=2 m(m+n-3)+\tau, \\
& (: /)=2 m(m+2 n-5)+2 \tau, \\
& (\cdot / /)=2 n(2 m+n-5)+2 \delta, \\
& (/ / /)=2 n(m+n-3)+\delta ;
\end{aligned}
$$

$(1,1,1)$

$$
\begin{aligned}
& (\quad: \quad)=\frac{1}{3}\left[2 m^{3}+6 m^{2} n-n^{3}-30 m^{2}-18 m n+13 n^{2}+84 m-42 n+(6 m+3 n-26) \tau\right], \\
& (\cdot /)=\frac{1}{6}\left[(m+n)\left(-(m+n)^{2}-7(m+n)+48\right)+4 m n(3 m+3 n-13)+2(3 m+3 n-20)(\delta+\tau)\right], \\
& (/ / \quad)=\frac{1}{3}\left[-m^{3}+6 m n^{2}+2 n^{3}+13 m^{2}-18 m n-30 n^{2}-42 m+84 n+(3 m+6 n-26) \delta\right]
\end{aligned}
$$


$(1,1,1,1)$

$$
\begin{aligned}
& (\cdot)=\frac{1}{6}\left\{2(m-3)(m-4)\left(n^{2}-m-n\right)+(n-3)(n-4)\left(m^{2}-m-n\right)\right. \\
& +4\left(m^{2}-11 m+28\right) \tau \quad+2\left(n^{2}-11 n+28\right) \delta \\
& \left.+(4(n-4)(m-4)-1)(2 \delta+\tau)+2 \delta^{2}+\tau^{2}\right\}, \\
& (/)=\frac{1}{6}\left\{\quad(m-3)(m-4)\left(n^{2}-m-n\right)+2(n-3)(n-4)\left(m^{2}-m-n\right)\right. \\
& +2\left(m^{2}-11 m+28\right) \tau \quad+4\left(n^{2}-11 n+28\right) \delta \\
& \left.+(4(n-4)(m-4)-1)(\delta+2 \tau)+\delta^{2}+2 \tau^{2}\right\}
\end{aligned}
$$

(2)

$$
\begin{aligned}
& (\therefore)=3 m+\iota, \\
& (: /)=2(3 m+\iota), \\
& (\cdot / /)=2(3 m+\iota), \\
& (/ / /)=3 m+\iota,
\end{aligned}
$$

$(2,1)$

$$
\begin{aligned}
& (:)=3\left(2 m n+n^{2}+4 m-10 n\right)+(2 m+n-14) \kappa \\
& (\cdot /)=2(3 m+\iota)(m+n-12)+24(m+n) \\
& (/ /)=3\left(m^{2}+2 m n-10 m+4 n\right)+(m+2 n-14) \iota
\end{aligned}
$$

$(2,1,1)$

$$
\begin{aligned}
(\cdot)= & (2 m+n-7)(6 \tau+(n-3) \kappa) \\
+ & ((m-n)(m+n-5)+\tau)(3 m+\iota-36) \\
+ & 12(m-n)(m+n-3) \\
(/)= & (m+2 n-7)(6 \delta+(m-3) \iota) \\
+ & ((n-m)(m+n-5)+\delta)(3 m+\iota-36) \\
+ & 12(n-m)(m+n-3)
\end{aligned}
$$

2, 2)

$$
\begin{aligned}
& (\cdot)=\frac{1}{2}(3 m+\iota)^{2}-3(3 m+\iota)-9 \tau-8 \delta \\
& (/)=\frac{1}{2}(3 m+\iota)^{2}-3(3 m+\iota)-8 \tau-9 \delta
\end{aligned}
$$

(3)

$$
\begin{aligned}
& (:)=6 n-4 m+3 \kappa=5 m-3 n+3 \iota \\
& (\cdot /)=10 n-8 m+6 \kappa=10 m-8 n+6 \iota \\
& (/ /)=5 n-3 m+3 \kappa=6 m-4 n+3 \iota
\end{aligned}
$$

$(1,3)$

( . $)=2\left(-4 m^{2}+3 m n+3 n^{2}+28 m-32 n\right)+3(2 m+n-13) \kappa$,

$(/)=2\left(3 m^{2}+3 m n-4 m^{2}-32 m+28 n\right)+3(m+2 n-13) \iota ;$

(4)

$$
\begin{aligned}
& (\cdot)=10 n-10 m+6 \kappa=8 m-8 n+6 \iota \\
& (/)=8 n-8 m+6 \kappa=10 m-10 n+6 \iota
\end{aligned}
$$

C. VI. 
Annex No. 7 (referred to, No. 93). question.

In connexion with De Jonquières' formula, I have been led to consider the following

Given a set of equations :

$$
\begin{aligned}
& \mathrm{a}=a \quad \text { (viz. } \mathrm{b}=b, \mathrm{c}=c, \& \mathrm{c} .) \text {, } \\
& \left.\begin{array}{rl}
a b= & a b \\
& +\left(\text { 11) } a \cdot b \left(\begin{array}{c}
\text { viz. } a c= \\
\quad
\end{array} \quad+(11) a c, \& c .,\right.\right. \text { and the like in all the subsequent equations }
\end{array}\right), \\
& a b c=a b c \\
& +(12)(a . b c+b \cdot a c+c \cdot a b) \\
& +(111) a \cdot b \cdot c \text {, } \\
& \text { abcd }=\quad a b c d \\
& +(13)(a . b c d+\& c .) \\
& +(22)(a b \cdot c d+\& c .) \\
& +(112)(a \cdot b \cdot c d+\& c .) \\
& +(1111) a . b . c . d \text {, }
\end{aligned}
$$

and so on indefinitely (where the $(\cdot)$ is used to denote multiplication, and $a b$, $a b c$, \&c., and also ab, abc, \&c. are so many separate and distinct symbols not expressible in terms of $a, b, c \& c ., \mathrm{a}, \mathrm{b}, \mathrm{c} \& \mathrm{c}$.), then we have conversely a set of equations

$$
\begin{aligned}
& a=\quad \mathrm{a} \text { (viz. } b=\mathrm{b}, \quad c=\mathrm{c} \& \mathrm{c} .) \text {, }
\end{aligned}
$$

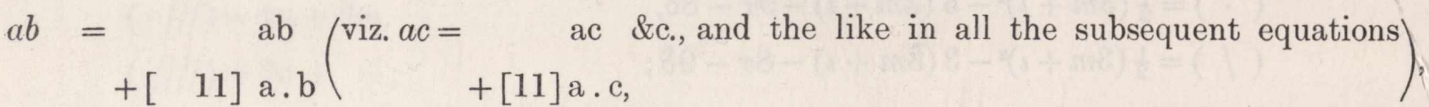

$$
\begin{aligned}
& a b c=a b c \\
& +\left[\begin{array}{ll}
12
\end{array}\right](\mathrm{a} \cdot \mathrm{bc}+\mathrm{b} \cdot \mathrm{ac}+\mathrm{c} \cdot \mathrm{ab}) \\
& +[111] \text { a.b.c, } \\
& a b c d=\quad \text { abcd } \\
& +[13](\mathrm{a} . \mathrm{bcd}+\& \mathrm{c} \text {. }) \\
& +\left[\begin{array}{ll}
22
\end{array}\right](\mathrm{ab} . \mathrm{cd}+\& \mathrm{c} .) \\
& +[112](\mathrm{a} \cdot \mathrm{b} \cdot \mathrm{cd}+\& \mathrm{c} .) \\
& +[1111] \text { a.b.c.d, }
\end{aligned}
$$


and so on; and it is required to find the relation between the coefficients ( ) and [ ]; we find, for example,

$$
\begin{aligned}
& \text { [11] }=-\quad \text { (11), } \\
& {[12]=-\quad(12) \text {, }} \\
& {[111]=\quad 3(11)(12)} \\
& \text { - (111), } \\
& \text { [13] }=-\quad(13) \text {, } \\
& {[22]=-\quad(22) \text {, }} \\
& {[112]=2(13)(12)} \\
& +\quad(22)(11) \\
& \text { - (112), } \\
& {[1111]=-12 \quad(13)(12)(11)} \\
& +4 \text { (13) (111) } \\
& \text { - } 3 \text { (22)(11)(11) } \\
& +6(112)(11) \\
& \text { - (1111); }
\end{aligned}
$$

and it is to be noticed that, conversely, the coefficients ( ) are given in terms of the coefficients [ ] by the like equations with the very same numerical coefficients; in fact from the last set of equations, this is at once seen to be the case as far as (112); and for the next term (1111) we have

$$
\begin{aligned}
& (1111)=+\mathrm{I} 2[13][12][11]=(\mathrm{I} 2-\mathrm{I} 2-\mathrm{I} 2=)-\mathrm{I} 2 \quad[13][12][11] \\
& -4[13]\{3[12][11]-[111]\} \quad+4[13][111] \\
& +3[22][11][11]+(3-6=\quad)-3[22][11][11] \\
& -6[11](2[13][12])+6[112][11] \\
& -[1111]\left\{\begin{array}{l}
+[22][11] \\
-[112]
\end{array}\right\} \\
& -\quad[1111]
\end{aligned}
$$

having the same coefficients $-12,+4,-3,+6,-1$ as in the formula for [1111] in terms of the coefficients ( ); it is easy to infer that the property holds good generally.

To explain the law for the expression of the coefficients of either set in terms of $33-2$ 
the other set, I consider, for example, the case where the sum of the numbers in the ( ), or [ ] is $=5$; and I form a kind of tree as follows:

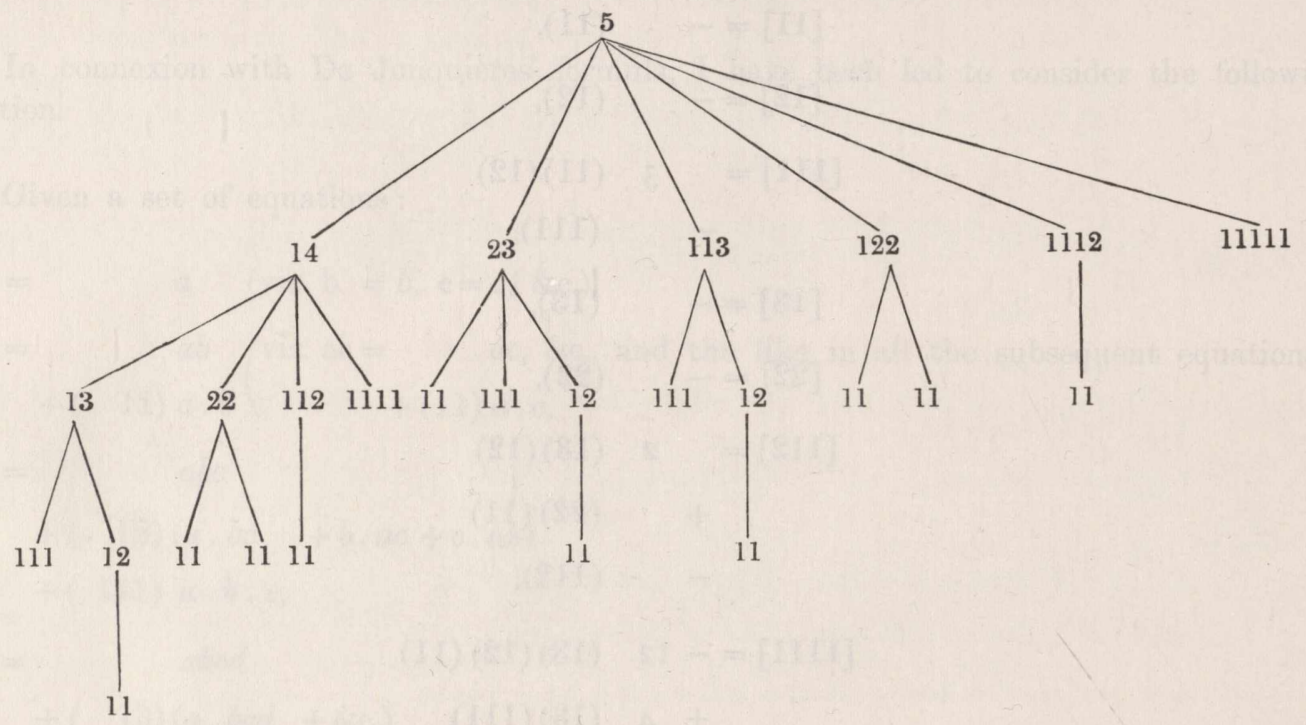

the formation of which is obvious; and I derive from it in the manner about to be explained the expressions for the coefficients [14], [23] \&c. in terms of the corresponding coefficients in ( ); viz. we have

$$
\begin{aligned}
& \text { [14] }=-\quad(14) \text {, } \\
& \text { [23] }=-\quad(23) \text {, } \\
& {[113]=2(14)(13)} \\
& +\quad \text { (23)(11) } \\
& \text { - (113), } \\
& {[122]=\quad(14)(22)} \\
& +2 \text { (23)(12) } \\
& \text { - (112), } \\
& {[1112]=-6(14)(13)(12)} \\
& \text { - } 3 \text { (14)(22)(11) } \\
& +3(14)(112) \\
& \text { - } 6 \text { (23)(12)(11) } \\
& +3 \text { (113)(12) } \\
& +\quad \text { I (23) (111) } \\
& +3 \text { (122)(11) } \\
& \text { - I (1112), }
\end{aligned}
$$




$$
\begin{aligned}
& {[11111]=+60 \quad(14)(13)(12)(11)} \\
& \text { - } 20 \text { (14) (13)(111) } \\
& \text { + } 15 \text { (14)(22)(11)(11) } \\
& \text { - } 30 \text { (14) (112)(11) } \\
& +5 \text { (14)(1111) } \\
& +30(23)(12)(11)(11) \\
& \text { - } \text { IO (23)(111)(11) } \\
& \text { - } 30 \quad(113)(12)(11) \\
& \text { + } 10 \text { (113)(111) } \\
& \text { - I } 5 \text { (122)(11)(11) } \\
& \text { + IO (1112)(11) } \\
& \text { - I (11111). }
\end{aligned}
$$

To form the symbolic parts, we follow each branch of the tree to each point of its course: thus from the branch 113 we have

$\begin{array}{lll}(113) & \text { belonging to } & {[113],} \\ (113)(111) & \# & {[11111],} \\ (113)(12) & \# & {[1112],} \\ (113)(12)(11) \quad \# & {[11111] ;}\end{array}$

viz. (113) belongs to [113]; (113)(111), read 11 (3 replaced by) 111, belongs to [11111]; (113) (12), read 11 (3 replaced by) 12, belongs to 1112 ; (113)(12)(11), read 11 (3 replaced by) 1 ( 2 replaced by) 11, belongs to [11111].

And observe that where (as, for example, with the symbol 122) there are branches derived from two or more figures, we pursue each such branch separately, and also all or any of them simultaneously to every point in the course of such branch or branches; thus for the branch 122 we have

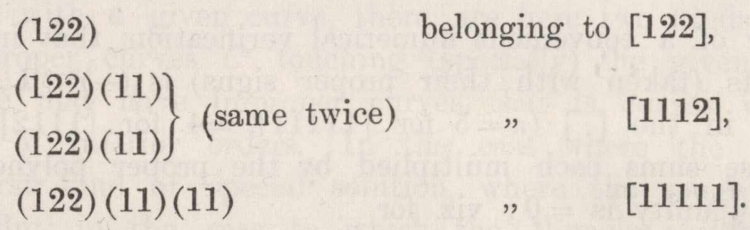

Similarly for the branch 23 we have

$\begin{array}{lcc}(23) & \text { belonging to } & {[23],} \\ (23)(111) & " & {[1112],} \\ (23)(12) & " & {[122],} \\ (23)(12)(11)(\text { same as } \text { infrà) } & " & {[1112],} \\ (23)(11)(111) & " & {[11111],} \\ (23)(11)(12) \text { (same as suprà) } & " & {[1112],} \\ (23)(11)(12)(11) & " & {[11111]}\end{array}$


We thus obtain the symbolic parts of the several expressions for [14], [23]... [11111] respectively: the sign of each term is + or - according as the number of factors in ( ) is even or odd; thus in the expression for [11111], the term (14) (13) (12) (11) has four factors, and is therefore + , the term (113)(12)(11) has three factors, and is therefore - .

The numerical coefficients are obtained as follows. There is a common factor derived from the expression in [ ] on the left-hand side of the equation; viz. for [11111], which contains five equal symbols, this factor is $1.2 .3 .4 .5,=120$; for [1112], which contains three equal symbols, it is $1.2 .3,=6$; and so on (for a symbol such as [11222] containing two equal symbols, and three equal symbols, the factor would be $1 \cdot 2 \cdot 1 \cdot 2 \cdot 3,=12$, and so in other similar cases). In any term on the righthand side of the equation, we must for a factor such as (11), which contains two equal symbols, multiply by $\frac{1}{2}$; for a factor such as (111), which contains three equal symbols, multiply by $\frac{1}{6}$, and so on. And in the case where a term (as, for example, the term $(122)(11)$ or $(23)(12)(11)$, vide suprà) occurs more than once, the term is to be taken account of each time that it occurs; or, what is the same thing, since the coefficient obtained as above is the same for each occurrence, the coefficient obtained as above is to be multiplied by the number of the occurrences of the term. For example, taking in order the several terms of the expression for [1112], the common factor is $=6$, and the several coefficients are

$$
6,6 \cdot \frac{1}{2}, 6 \cdot \frac{1}{2}, 6 \cdot \frac{1}{2} \times 2,6 \cdot \frac{1}{2}, 6 \cdot \frac{1}{6}, 6 \cdot \frac{1}{2} \cdot \frac{1}{2} \times 2,6 \cdot \frac{1}{6}
$$

and similarly in the expression for [111i1] the common factor is 120 , and the coefficients taken in order are

$$
120 \cdot \frac{1}{2}, \quad 120 \cdot \frac{1}{6}, \quad 110 \cdot \frac{1}{2} \cdot \frac{1}{2} \cdot \frac{1}{2}, \& c \text {, }
$$

without there being in this case any coefficient with a factor arising from the plural occurrence of the term.

The foregoing result was established by induction, and I have not attempted a general proof.

I observe by way of a convenient numerical verification, that in each equation the sum of the coefficients (taken with their proper signs) is $(-)^{n-1} 1.2 \ldots(n-1)$; if $n$ be the number of parts in the [ ] $(n=5$ for [11111], =4 for [1112] \&c.), and moreover, that the sum of these sums each multiplied by the proper polynomial coefficient and the whole increased by unity is $=0$; viz. for

$$
\text { [14], [23], [113], [122], [1112], [11111], }
$$

the sums of the coefficients are

and we have

$$
-1,-1, \quad+2, \quad+2, \quad-6, \quad+24 \text { respectively. }
$$

$$
1+5(-1)+10(-1)+10(2)+15(2)+10(-6)+1(24),=75-75,=0 .
$$

If we have any five distinct things $(a, b, c, d, e)$, then the polynomial coefficients $5,10,10,15,10,1$ denote respectively the number of ways in which these can be partitioned in the forms $14,23,113,122,1112,11111$ respectively, and the last-mentioned theorem is thus a theorem in the Partition of Numbers. 